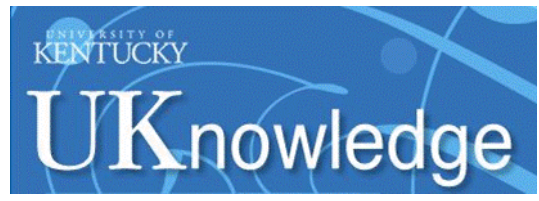

University of Kentucky UKnowledge

\title{
$2-2017$
}

\section{Obesity: Current and Potential Pharmacotherapeutics and Targets}

Vidya Narayanaswami

University of Kentucky, vidyan83@gmail.com

Linda P. Dwoskin

University of Kentucky, Idwoskin@email.uky.edu

Right click to open a feedback form in a new tab to let us know how this document benefits you. Follow this and additional works at: https://uknowledge.uky.edu/ps_facpub

Part of the Pharmacy and Pharmaceutical Sciences Commons 


\section{Obesity: Current and Potential Pharmacotherapeutics and Targets}

Digital Object Identifier (DOI)

https://doi.org/10.1016/j.pharmthera.2016.10.015

\section{Notes/Citation Information}

Published in Pharmacology \& Therapeutics, v. 170, p. 116-147.

(c) 2016 Elsevier Inc. All rights reserved.

This manuscript version is made available under the CC-BY-NC-ND 4.0 license https://creativecommons.org/licenses/by-nc-nd/4.0/.

The document available for download is the author's post-peer-review final draft of the article. 
Published in final edited form as:

Pharmacol Ther. 2017 February ; 170: 116-147. doi:10.1016/j.pharmthera.2016.10.015.

\title{
Obesity: Current and Potential Pharmacotherapeutics and Targets
}

\author{
Vidya Narayanaswami and Linda P. Dwoskin \\ Department of Pharmaceutical Sciences, College of Pharmacy, University of Kentucky, Lexington, \\ KY, USA 40536
}

\section{Abstract}

Obesity is a global epidemic that contributes to a number of health complications including cardiovascular disease, type 2 diabetes, cancer and neuropsychiatric disorders.

Pharmacotherapeutic strategies to treat obesity are urgently needed. Research over the past two decades has increased substantially our knowledge of central and peripheral mechanisms underlying homeostatic energy balance. Homeostatic mechanisms involve multiple components including neuronal circuits, some originating in hypothalamus and brain stem, as well as peripherally-derived satiety, hunger and adiposity signals that modulate neural activity and regulate eating behavior. Dysregulation of one or more of these homeostatic components results in obesity. Coincident with obesity, reward mechanisms that regulate hedonic aspects of food intake override the homeostatic regulation of eating. In addition to functional interactions between homeostatic and reward systems in the regulation of food intake, homeostatic signals have the ability to alter vulnerability to drug abuse. Regarding the treatment of obesity, pharmacological monotherapies primarily focus on a single protein target. FDA-approved monotherapy options include phentermine (Adipex-P®), orlistat (Xenical $\left.{ }^{\circledR}\right)$, lorcaserin (Belviq ${ }^{\circledR}$ ) and liraglutide (Saxenda $\left.{ }^{\circledR}\right)$. However, monotherapies have limited efficacy, in part due to the recruitment of alternate and counter-regulatory pathways. Consequently, a multi-target approach may provide greater benefit. Recently, two combination products have been approved by the FDA to treat obesity, including phentermine/topiramate (Qsymia ${ }^{\circledR}$ ) and naltrexone/bupropion (Contrave $\left.{ }^{\circledR}\right)$. The current review provides an overview of homeostatic and reward mechanisms that regulate energy balance, potential therapeutic targets for obesity and current treatment options, including some candidate therapeutics in clinical development. Finally, challenges in anti-obesity drug development are discussed.

\footnotetext{
Corresponding Author: Linda P. Dwoskin, Ph.D., Department of Pharmaceutical Sciences, University of Kentucky, College of Pharmacy, 789 South Limestone, 465 Biological Pharmaceutical Complex, Lexington, KY 40536-0596, Phone: (859) 257-4743; FAX: (859) 257-7585, ldwoskin@email.uky.edu.

Publisher's Disclaimer: This is a PDF file of an unedited manuscript that has been accepted for publication. As a service to our customers we are providing this early version of the manuscript. The manuscript will undergo copyediting, typesetting, and review of the resulting proof before it is published in its final citable form. Please note that during the production process errors may be discovered which could affect the content, and all legal disclaimers that apply to the journal pertain.

CONFLICT OF INTEREST

The authors declare that there are no conflicts of interest.
} 


\section{Keywords}

homeostasis; obesity; pharmacotherapy; reward

\section{Introduction}

Obesity is a complex medical condition characterized by excessive, abnormal fat accumulation as a result of increased intake of energy-dense foods and decreased physical activity (WHO, 2015). The dramatic rise in obesity is attributed to genetic susceptibility, environmental factors such as availability of energy dense foods and sedentary life style lacking adequate physical activity. The prevalence of obesity in the Unites States during 2009-2010 was about 36\% among adult men, 36\% among adult women, and 17\% among children and adolescents (National Health and Examination Survey, 2010). Projections based on current trends suggest an increase in obesity prevalence by 2050 to $60 \%$ in adult men, $40 \%$ in adult women and $25 \%$ in children (CDC Report, 2014). The rise in global obesity rates over the last three decades has been substantial, presenting a major public health epidemic in both developed and developing countries (Ng et al., 2014). A systematic analysis in 2013 of the global burden of disease revealed that more than $50 \%$ of the world's 671 million obese individuals live in 10 countries (ranked from most to least): United States, China, India, Russia, Brazil, Mexico, Egypt, Germany, Pakistan and Indonesia (Ng et al., 2014). Once considered a problem predominantly in western countries, obesity has become a global epidemic.

Obesity is a major contributor to the metabolic syndrome, a constellation of metabolic abnormalities including increased blood pressure, high blood sugar, excess body fat around the waist, high serum triglycerides, and low levels of high-density lipoproteins (Mokdad et al., 2001; Bloomgarden, 2002; Montani et al., 2002; Eckel et al., 2005; Grundy et al., 2005). The metabolic syndrome increases the risk for severe health problems including cardiovascular disease, type 2 diabetes and stroke (Eckel et al., 2005). Compared to nonobese women, obese women exhibit 60-fold greater probability of type 2 diabetes (Colditz et al., 1990). Overweight and obese men exhibit increased risk for ischemic and hemorrhagic stroke (Kurth et al., 2002).

In addition to the metabolic syndrome, obesity also increases risk for certain types of cancer. As examples, the contribution of obesity to cancer is as high as $40 \%$ for endometrial and esophageal adenocarcinoma (NCI, 2012). Current trends indicate that by 2030, there will be an additional 500,000 cases of cancer associated with obesity in the United States.

Furthermore, the prevalence of neuropsychiatric disorders, particularly, dementia, depression and anxiety, is increased in obesity (Petry et al., 2008). Escalating rates of obesity and concomitant life-threatening disorders underscore the need for safe and effective strategies to treat this complex medical condition.

Multiple approaches and strategies are used for the treatment of obesity including lifestyle modifications (making healthy dietary choices and increasing exercise), bariatric surgery and pharmacotherapy (Polonsky and Klein, 2008). A healthy diet and increase in exercise are beneficial for both the prevention and treatment of obesity. Nonetheless, lifestyle 
modifications require self-discipline and persistence to ensure and maintain the necessary beneficial weight loss. Moreover, effective lifestyle management of obesity results from a partnership between a highly motivated patient and a committed team of health professionals that may include a physician, a psychologist, psychiatrist, physical and exercise therapists, dietitians and other sub-specialists, depending on the patient's comorbidities (Polonsky and Klein, 2008).

Importantly, lifestyle management is just one component of a comprehensive approach to treat obesity and associated metabolic abnormalities (Smith et al., 2011). Surgical approaches, such as bariatric surgery, produce significant weight loss and ameliorate associated cardiovascular complications and type 2 diabetes in morbidly obese patients (Abdeen and le Roux, 2015). However, surgical procedures are invasive, expensive and have their own inherent risks and side-effects, including increased alcohol use, which suggests that these procedures facilitate an addiction transfer or exchange of palatable food reinforcers for an alternate reinforcer such as alcohol (Lent et al., 2013; Polston et al., 2013). Durability of diabetes remission and incident relapse following bariatric surgery was assessed from 1995 to 2008 in a large, population-based study of three integrated health care delivery systems in the United States (Arterburn et al., 2013). Although bariatric surgery resulted in complete diabetes remission within five years in $68 \%$ of severely obese adults, one-third of the individuals undergoing surgery relapsed within five years of initial remission (Arterburn et al., 2013). Further investigation is needed to understand the mechanisms underlying the cardio-metabolic effects of bariatric surgery and the long-term efficacy and safety of these procedures.

Given the limitations of lifestyle interventions and bariatric surgery, pharmacotherapeutic approaches for the treatment of obesity are important options. During the past 20 years, several anti-obesity drugs have been discovered, marketed and subsequently withdrawn from the market. Despite showing efficacy during initial stages of treatment, therapeutics for obesity have been accompanied by adverse side-effects following long-term use.

Nevertheless, robust escalations in obesity and associated health complications constitute major driving forces for the discovery of novel targets and for the development of safe and effective weight loss therapeutics. The current review discusses potential therapeutic targets for obesity and provides an overview of current FDA-approved anti-obesity drugs, as well as several therapeutic candidates under clinical investigation. With respect to the discovery of novel therapeutic targets, employment of powerful molecular and genetic tools has increased our knowledge of central and peripheral mechanisms underlying homeostatic energy balance. The current review discusses interactions between homeostatic and nonhomeostatic hedonic mechanisms underlying excessive food intake. Centrally and perimpherally derived factors that regulate homeostatic and non-homeostatic hedonic mechanisms underlying food intake are summarized in Figure 1.

\section{Homeostatic regulation of energy balance}

Obesity results from a long-term positive energy balance, that is, increased food intake and decreased energy expenditure (Spiegelman and Flier, 2001). Homeostasis is the maintenance of equilibrium of energy balance through adjustments in physiological processes. Both 
central and peripheral mechanisms are involved in the maintenance of body weight set point. Set point is a descriptor of long-term weight maintenance that involves coordinated adjustments in both intake and expenditure of energy that serve to stabilize an individual's weight at a specified level and to resist displacement from this level (Keesey and Hirvonen, 1997). Distinct neuronal circuits and signaling molecules within the hypothalamus and brain stem regulate energy balance. Peripherally-derived satiety, hunger and adiposity signals are integrated within hypothalamic and brainstem circuits to regulate feeding and metabolism. Satiety is a state of feeling full after eating such that eating behavior is inhibited subsequently (Blundell, 1991). The desire to eat in response to sensory stimulation is generally considered to represent appetite (Blundell, 1991). Biological stimuli leading to feeding are considered to represent hunger, which is triggered by i) a contracting empty stomach, ii) an empty small intestine, iii) the hormone ghrelin produced between meals and iv) other signals that affect the brain (Read, 1992; Sanger et al., 2010). Hunger initiates the motivation to seek and consume food (Harrold et al., 2012). Adiposity signals include feedback signals that are generated in response to food intake and accumulation of fat mass. Importantly, the majority of these central and peripheral mechanisms serve as potential therapeutic targets for obesity.

\subsection{Central mechanisms}

Hypothalamic neuronal circuits are implicated as the primary regulators of energy homeostasis and include the arcuate nucleus, which is located ventrally around the third ventricle of the brain (Wynne et al., 2005; Morrison and Berthoud, 2007). Arcuate nucleus neurons are called 'first order' neurons since peripheral adiposity factors act directly on these neurons (Hillebrand et al., 2002). Circulating adiposity signals such as leptin and insulin are transported actively across the blood brain barrier and bind to respective receptors on arcuate cell bodies. While the cell bodies of hypothalamic secretory neurons are localized in areas protected by the blood-brain barrier, their axon terminals are localized in the median eminence, which lacks a blood-brain barrier, thereby allowing central nervous system neurons to secrete hormones into the blood stream (Peruzzo et al., 2000). Activation of distinct neuronal populations within the arcuate nucleus either stimulates or inhibits food intake. From the arcuate nucleus, neurons project to 'second order' neurons in the paraventricular nucleus, lateral hypothalamus, perifornical area and dorsomedial hypothalamus (Schwartz et al., 2000). Second order neurons project to the nucleus tractus solitarius (NTS) in the brainstem and the dorsomotor nucleus of the vagus (Hillerband et al., 2002). NTS and area postrema integrate afferent signals and relay them to other regulatory brain centers, including the arcuate nucleus (Berthoud, 2002; Harrold et al., 2012). NTS lesion results in elevated consumption of palatable foods (Hyde and Miselis, 1983). Thus, hypothalamus and brain stem contain distinct neuronal circuits, signaling molecules and reciprocal connections regulating energy homeostasis. Previous reviews have provided comprehensive details regarding central mechanisms implicated in the regulation of energy homeostasis (Parker and Bloom, 2012; Williams and Elmquist, 2012; Sohn, 2015). The current section briefly introduces these mechanisms and provides insight regarding potential therapeutic targets for the treatment of obesity. 
2.1.1. Arcuate nucleus: Orexigenic and anorexigenic systems-Within the arcuate nucleus, one subpopulation of neurons expresses orexigenic peptides including neuropeptide Y (NPY) and agouti-related protein (AgRP). NPY is co-expressed with AgRP in the arcuate nucleus, and these neurons project to second order neurons in paraventricular nucleus, ventral medial hypothalamus, dorsomedial hypothalamus and lateral hypothalamus (Elias et al., 1998; Hahn et al., 1998; Kalra et al., 1999). Repeated bilateral injection of NPY ( $235 \mathrm{pmol} ; 3$ times/day for 10 days) into the paraventricular nucleus of rats resulted in increased food intake, body weight gain and body fat accumulation (Stanley et al., 1986). Orexigenic effects of NPY are mediated predominantly by G-protein coupled receptors (GPCRs), Y1 and Y5 within the paraventricular nucleus (Sohn et al., 2013). Also, $\gamma$ aminobutyric acid (GABA) is colocalized with NPY and AgRP in the arcuate nucleus (Broberger, et al., 1998; Cowley, et al., 2001). AgRP neurons express receptors for peripheral hormonal signals including insulin, leptin and ghrelin (Elmquist, et al., 1998; Willesen et al., 1999). Acute intracerebroventricular (ICV) injection of AgRP (1.5-15.0 $\mu \mathrm{g} /$ $10 \mu \mathrm{l})$ increased in a dose-dependent manner cumulative food intake and body weight in rats (Ebihara et al., 1999). Also, leptin ( $2 \mu \mathrm{g}$, ICV) inhibition of food intake and body weight was reversed dose-dependently by co-injection of AgRP (0.15-1.5 $\mu \mathrm{g}$, ICV).

Another subpopulation of arcuate nucleus neurons expresses anorexigenic peptides including a-melanocyte stimulating hormone (a-MSH) and cocaine and amphetamine regulated transcript (CART) (Mains and Eipper, 1980; Elias et al., 1998). The anorexigenic role of CART is emphasized by the demonstration that CART-deficient mice fed a high-fat diet showed increased food consumption, body weight and fat mass relative to wild-type littermates (Asnicar et al., 2001). Once cleaved from precursor hormone proopiomelanocortin (POMC), a-MSH binds to melanocortin-4 receptors (MC4Rs) (Eipper and Mains, 1980). Acutely, a-MSH ( $1 \mathrm{nmol} / 10 \mu \mathrm{l}, \mathrm{ICV})$ decreases food intake in rats (Rossi et al., 1998). Mutations in MC4R are associated with morbid obesity in humans (Krude et al., 1998; Yeo et al., 1998), consistent with findings that MC4R deficient mice exhibit an obesity phenotype (Huszar et al., 1997). MC4R signaling is necessary for Roux-en-Y gastric bypass surgery-induced weight loss, decreases in food intake and food preference, and increases in energy expenditure (Hatoum et al., 2012). Compared to wild-type mice, MC4R knockout mice exhibited decreased weight loss after Roux-en-Y gastric bypass surgery (Hatoum et al., 2012). Chronic treatment with a highly-selective, novel, MC4R peptide agonist, RM-493 (Ac-Arg-Cys(1)-D-Ala-His-D-Phe-Arg-Trp-Cys(1)-NH2; $0.5 \mathrm{mg} / \mathrm{kg} / \mathrm{day}$ for $8 \mathrm{wk}$, subcutaneous (SC)), transiently decreased (35\%) food intake, resulted in weight loss $(13.5 \%)$, decreased adiposity, and improved glucose tolerance in a non-human primate model of diet-induced obesity (DIO) (Kievit et al., 2013). Currently, RM-493 is in Phase II clinical trials to evaluate its safety and efficacy in obese human subjects. Taken together, arcuate nucleus dysfunction, including increased activity of NPY/AgRP versus POMC neurons, at least in part, underlies some forms of obesity, and moreover, NPY, AgRP, MC4R and CART may serve as viable targets for obesity therapeutics.

2.1.2. Lateral hypothalamus: Orexigenic systems—Within the lateral hypothalamus/perifornical area, second-order neurons express orexigenic neuropeptides, orexin (hypocretin) and melanin-concentrating hormone (MCH) (Qu et al., 1996; de Lecea 
et al., 1998; Sakurai et al., 1998). Prepro-orexin, a 130 amino acid precursor peptide, is processed to orexin A and orexin B, which are 33 and 28 amino acid peptides, respectively (Sakurai et al., 1998). Administration of orexin A and B ( 3 and $30 \mathrm{nmol} / 5 \mu \mathrm{l}$, ICV) promotes hyperphagia in rats (Sakurai et al., 1998). Orexins activate two GPCR subtypes, the orexin-1 receptor that is highly expressed in the ventral medial hypothalamus and the orexin-2 receptor that is expressed predominantly in the paraventricular nucleus (Trivedi et al., 1998). Orexin A binds with high affinity to both orexin-1 and -2 receptors, whereas orexin B selectively binds to orexin-2 receptors (Sakurai et al., 1998). Prepro-orexin knockout mice and orexin-neuron ablated transgenic mice exhibit a hypophagic phenotype (Hara et al., 2001; Willie et al., 2001). Repeated intraperitoneal (IP) administration of the orexin-1 receptor antagonist, SB334867-A (1-(2-methyl-1,3-benzoxazol-6-yl)-3-(1,5-naphthyridin-4yl) urea hydrochloride; $30 \mathrm{mg} / \mathrm{kg}$ for $2 \mathrm{wk}$, IP) to genetically obese ob/ob (leptin-deficient) mice decreased cumulative food intake, body weight, fat mass gain, fasting blood glucose and plasma insulin levels, compared to vehicle-treated ob/ob mice (Haynes et al., 2002). Also, acute treatment with SB334867-A increased energy expenditure in ob/ob mice, determined by indirect calorimetry.

Similar to orexins, central administration of $\mathrm{MCH}(5$ or $30 \mu \mathrm{g}$, ICV) increased food intake in rats (Qu et al., 1996). MCH acts through two GPCRs, MCH1 and MCH2 receptors (Pissios et al., 2006). In mammals, MCH1 and MCH2 receptors are expressed in frontal cortex, amygdala, nucleus accumbens, arcuate nucleus and ventral medial hypothalamus (Hervieu et al., 2000). However, non-primate animal models lack MCH2 receptors, limiting their usefulness in understanding the physiological role of MCH2 (Tan et al., 2002). MCH1 receptor knockout mice are lean, resistant to DIO, exhibit hyperactivity and exhibit hypermetabolism compared to wild-type controls (Chen et al., 2002; Marsh et al., 2002; Pissios, 2009). Taken together, orexin-1 receptor antagonists and MCH1 receptor antagonists appear to have therapeutic potential for the treatment of obesity.

\subsubsection{Paraventricular nucleus: Anorexigenic systems-In contrast to lateral} hypothalamus, paraventricular nucleus neurons express anorexigenic peptides including the peptide hormones, thyrotropin-releasing hormone and corticotrophin-releasing hormone, as well as brain derived neurotrophic factor (BDNF; Fekete et al., 2000; Kernie et al., 2000; Sarkar and Lechan, 2003). Central administration of corticotrophin-releasing hormone in rats (3 mg/rat, ICV) reduced food intake, compared to vehicle-treated rats (Vergoni et al., 1999). Also, thyrotropin-releasing hormone (4, 16 and $64 \mathrm{mg} / \mathrm{kg}$ twice/day, SC) dosedependently decreased food intake in rats (Choi et al., 2002). The effect of BDNF was assessed on NPY-induced feeding in rats (Wang et al., 2007). The paraventricular nucleus is an integral site for the anorectic effects of BDNF. NPY (100 pmol/ $/ 0.5 \mu \mathrm{l})$ was infused directly into the rat paraventricular nucleus $4 \mathrm{~h}$ after infusion of BDNF $(0.1-0.5 \mu \mathrm{g} / 0.5 \mu \mathrm{l})$. NPY increased food intake, and BDNF $(0.3$ and $0.5 \mu \mathrm{g})$ decreased NPY-induced feeding by $56 \%$ and $82 \%$, respectively, compared to the NPY-infused controls.

In a study using agouti lethal yellow mutant mice ( $A^{y}$ ), ectopic expression of the agouti protein (a homologue of AgRP) produced hyperphagia and obesity (Miller et al., 1993). Importantly, the phenotype of the MC4R null mutant mice is identical to that of the $A^{y}$ mutant mice (Miller et al., 1993). Moreover, agouti protein is reported to be an antagonist of 
MC4R, but not of the melanocortin-3 receptor (Lu et al., 1994; Huszar et al., 1997; Chen et al., 2000). In $\mathrm{A}^{\mathrm{y}}$ mutant mice fed a high-fat diet, which have disrupted MC4R signaling, BDNF (200 ng/ $\mu$ l for 14 days, ICV) suppressed daily food intake and body weight gain, compared to the vehicle-treated control mice (Xu et al., 2003). These results suggest that BDNF may regulate energy homeostasis downstream of MC4R. Additional animal and human studies exploring these systems may provide novel targets for obesity drug discovery.

2.1.4. Hindbrain mechanisms-Hindbrain neurons integrate behavioral, autonomic and endocrine responses to regulate energy balance (Grill and Hayes, 2012). Within the brainstem, the dorsal vagal complex plays an important role in relaying peripheral signals via vagal afferent fibers from the gut to the hypothalamus (Bailey, 2008). The dorsal vagal complex consists of the dorsal motor nucleus of vagus, area postrema and NTS (Stanley et al., 2005). The NTS is considered an integral part of the brain stem that receives inputs and processes peripherally-derived energy-status signals such as leptin, ghrelin, and glucose as well as signals from hypothalamic neurons (paraventricular nucleus, lateral hypothalamus and arcuate nucleus) (Grill and Hayes, 2012). Additionally, NTS neurons in caudal regions project to vagal efferent neurons in the dorsal motor nucleus of vagus that regulates parasympathetic gastrointestinal responses, including insulin secretion and gastric emptying, and project to other subregions of the hypothalamus that regulate neuroendocrine responses (Grill and Hayes, 2012).

Monoamine neurotransmitters, norepinephrine (NE) and serotonin (5-HT), are synthesized in brain stem and regulate food intake (Schwartz et al., 2000). NE is synthesized by discrete neuronal populations in brain stem, including the dorsal vagal complex and the locus coeruleus (Foote et al., 1983; Palkovits, 1999). Neuronal fibers from these areas project to the hypothalamus, thalamus, cortex and spinal cord (Foote et al., 1983; Palkovits, 1999). Genetically obese ob/ob mice exhibit increased NE levels in paraventricular nucleus and lateral hypothalamus, compared to lean littermate controls, suggesting a role for hypothalamic NE in obesity (Oltmans, 1983). Repeated NE injection (20 nmoles, 4 times/ day) into the paraventricular nucleus promotes hyperphagia and body weight gain in rats (Leibowitz et al., 1984). NE is an agonist at a 1- and a2-adrenoceptors (Wellman, 2000). Radioligand binding studies reveal the presence of both a 1- and a 2-adrenoceptors within the paraventricular nucleus, and the stimulatory effect of NE on eating is linked to activation of a 2-adrenoceptors (Leibowitz et al., 1982). Intra-paraventricular nucleus injection of either NE (40 nmol) or the a2-adrenoceptor agonist, clonidine ( $20 \mathrm{nmol})$, increased feeding in rats, compared to controls (Goldman et al., 1985). Further, hyperphagia induced by NE $(40 \mathrm{nmol})$ was inhibited dose-dependently by pretreatment with a a2-adrenoceptor antagonist, yohimbine (12.5-200 nmol), supporting an orexigenic role of paraventricular nucleus a2-adrenoceptors. Expression of a2-adrenoceptors in paraventricular nucleus is under circadian control, with increased expression at dark phase onset, when feeding behavior in rodents is increased (Wellman, 2000). In contrast to the hyperphagic effect of a2-adrenoceptor stimulation, injection of a1-adrenoceptor agonist, cirazoline (3-24 nmol) into paraventricular nucleus suppressed food intake in rats, compared to control (Davies and Wellman, 1992). Taken together, these results suggest that the relative balance of a1-and 
a2-adrenoceptors within the paraventricular nucleus modulates the impact of various adrenergic agonists on feeding.

With respect to the 5-HT system, raphe nucleus cell bodies in caudal brainstem project widely throughout brain, and activation of 5-HT receptors suppresses food intake (Leibowitz et al., 1988). Seven families of 5-HT receptors (5- $\left.\mathrm{HT}_{1-7}\right)$ comprised of at least 14 distinct subtypes have been described; $5-\mathrm{HT}_{1 \mathrm{~B}}, 5-\mathrm{HT}_{2} \mathrm{C}$ and $5-\mathrm{HT}_{6}$ are the primary subtypes implicated in energy balance (Garfield and Heisler, 2009). Fenfluramine, an indirect 5-HT agonist that releases 5-HT from vesicular stores and reverses 5-HT transporter function, was approved in combination with phentermine, a NE transporter inhibitor, for the treatment of obesity (Ioannides-Demos et al., 2011). This drug combination was withdrawn from the market due to adverse cardiovascular side-effects. Sibutramine, a dual 5-HT and NE uptake inhibitor, was found to be effective in the treatment of obesity (Ioannides-Demos et al., 2011). Sibutramine was withdrawn from the market also due to cardiovascular side-effects including increased systolic and diastolic blood pressure and heart rate (Scheen, 2011).

Genetic studies reveal the role of specific 5-HT receptor subtypes in the development of obesity. In contrast to $5-\mathrm{HT}_{2 \mathrm{C}}$ receptors, $5-\mathrm{HT}_{6}$ receptor activation promotes obesity, since $5-\mathrm{HT}_{6}$ receptor knockout mice exhibit decreased food intake and body weight gain upon exposure to a high-fat diet for $11 \mathrm{wk}$ (Frassetto et al., 2008). In DIO rats, the 5-HT 6 receptor antagonist, PRX-07034 ( $N$-[1-(5-chloro-2,3-dimethoxyphenyl)ethyl]-2-methylsulfonyl-5piperazin-1-ylaniline; $10 \mathrm{mg} / \mathrm{kg}, 2$ times/day for $6 \mathrm{wk}$; IP), reduced food intake throughout treatment and decreased (12.7\%) body weight (Gannon et al., 2006). Body composition analysis revealed that the PRX-07034-induced reduction in body weight was the result of a selective reduction in fat mass with minimal effects on either body water or protein content. Consistent with a marked decrease in white adipocyte fat mass, plasma leptin was decreased by more than $75 \%$ in the PRX-07034-treated rats compared to the vehicle control. In a randomized, double-blind, placebo-controlled Phase Ib clinical trial, PRX-07034 (600 mg, twice/day) produced significant weight loss in healthy obese patients (Heal et al., 2012). Phase II and III clinical trials will provide further information regarding its safety and efficacy as a treatment of obesity. Thus, pharmacotherapies targeting specific monoamine receptor subtypes may be beneficial for the treatment of obesity.

\subsection{Endocannabinoids: Central and peripheral regulation of energy balance}

Endocannabinoids are implicated in the central and peripheral regulation of energy balance. Anandamide and 2-arachidonylglycerol (2-AG) are the primary endocannabinoids in brain (Freund et al., 2003). Upon release by postsynaptic neurons, these molecules act as retrograde messengers activating presynaptic cannabinoid-1 (CB1) receptors to inhibit synaptic transmission by either excitatory glutamatergic or inhibitory GABAergic neurons (Freund et al., 2003). In contrast to CB1 receptors, cannabinoid-2 (CB2) receptors are highest in immune cells (Maresz et al., 2007). The role of endocannabinoids in the regulation of homeostatic energy balance is evident from CB1-positive axonal innervation of the arcuate nucleus, paraventricular nucleus, dorsomedial hypothalamus and ventral medial hypothalamus (Wittmann et al., 2007). Importantly, corticotrophin releasing hormone mRNA is increased in paraventricular nucleus of CB1 knockout mice (Cota et al., 2007). 
Mice deficient in fatty acid amide hydrolase, the enzyme responsible for anandamide metabolism, exhibit decreased levels of CART-immunoreactivity in the arcuate nucleus, dorsomedial hypothalamus and paraventricular nucleus, compared to wild-type control (Osei-Hyiaman et al., 2005a). Moreover, treatment of fatty acid amide hydrolase deficient mice with CB1 inverse agonist, rimonabant ( $3 \mathrm{mg} / \mathrm{kg} /$ day for 7 days, IP) increased CART in these brain regions compared to wild-type controls (Osei-Hyiaman et al., 2005a).

Endocannabinoid stimulation of central CB1 receptors results in orexigenic effects. Anandamide $(50 \mathrm{ng} / 0.5 \mu \mathrm{l})$ infused into the ventral medial hypothalamus of pre-satiated rats produced hyperphagia that was attenuated by pretreatment with rimonabant $(30 \mu \mathrm{g} / 0.5 \mu \mathrm{l})$ (Jamshidi and Taylor, 2001). Selective CB1 receptor deletion in mouse forebrain results in DIO resistance (Quarta et al., 2010). Consistent with these results, transgenic mice that overexpress monoacylglycerol lipase, the enzyme that deactivates 2-AG, in forebrain are DIO resistant and express high levels of thermogenic proteins in brown adipose tissue, compared to wild-type control (Jung et al., 2012). These mutant mice are hypersensitive to $\beta 3$-adrenergic-stimulated thermogenesis that is normalized by a single dose of JZL184 (4nitrophenyl-4-[bis(1,3-benzodioxol-5-yl)(hydroxy)methyl]piperidine-1-carboxylate; 16 $\mathrm{mg} / \mathrm{kg}$, IP), an irreversible monoacylglycerol lipase inhibitor. JZL184 normalization of $\beta 3$ adrenergic-stimulated thermogenesis was reversed by rimonabant $(10 \mathrm{mg} / \mathrm{kg}$, IP), indicating that impaired forebrain 2-AG signaling at CB1 receptors underlies enhanced thermogenesis in these mutant mice.

Alterations in energy status modulate the balance between hypothalamic excitatory and inhibitory synaptic transmission and synaptic plasticity to facilitate homeostatic adaptation (Cristino et al., 2013). In obesity, CB1-expressing excitatory vs. inhibitory inputs to orexinA containing neurons in lateral hypothalamus are altered. In lean mice, these inputs are mostly excitatory, whereas in ob/ob and DIO mice, orexinergic neurons receive predominantly inhibitory CB1-expressing inputs (Cristino et al., 2013). To determine if the altered synaptology of orexigenic neurons was due to leptin deficiency, glutamatergic and GABAergic inputs to orexigenic neurons were analyzed in ob/ob and wild-type mice following leptin $(5 \mathrm{mg} / \mathrm{kg}$, IP). Increased vesicular glutamate transporter- 2 and decreased vesicular GABA transporter were found in lateral hypothalamus of ob/ob mice after leptin, resembling vehicle-injected wild-type mice (Cristino et al., 2013). Effects of leptin were abolished by a leptin receptor antagonist, indicating that leptin reverses remodeling of orexigenic neurons in ob/ob mice. Thus, in obesity, endocannabinoid neuromodulatory control is altered as a result of leptin-dependent reorganization of glutamatergic and GABAergic synapses in hypothalamus.

In Europe, rimonabant was used as an adjunct to diet and exercise for the treatment of obesity (Christensen et al., 2007). However, rimonabant ( $20 \mathrm{mg} /$ day, oral) increased the risk of psychiatric events including depressed mood disorders, anxiety and suicidal ideation, resulting in its withdrawal from the European market (European Medicines Agency, 2008). While lacking the deleterious psychiatric effects of rimonabant, NESS0327 (8-chloro-1-(2,4dichlorophenyl)-N-piperidin-1-yl-5,6-dihydro-4H-benzo[2,3]cyclohepta[2,4-b]pyrazole-3carboxamide, $0.03-0.3 \mathrm{mg} / \mathrm{kg}$, IP), a neutral CB1 antagonist, decreased food intake and body weight in rats (Meye et al., 2013). In contrast to rimonabant, NESS0327 did not alter 
GABAergic and glutamatergic postsynaptic currents in ventral tegmental area (VTA) neurons or in basolateral amygdala. NESS0327 $(0.1 \mathrm{mg} / \mathrm{kg}$, IP) was not anxiogenic and blocked the anxiogenic response as well as decreases in motivation for sucrose produced by rimonabant (1 mg/kg, IP; Meye et al., 2013). Results suggest that alternate mechanisms may underlie hypophagic effects of NESSO327.

Interestingly, co-administration of rimonabant $(0.3 \mathrm{mg} / \mathrm{kg}$, IP) and an $\mathrm{MCH} 1$ receptor antagonist, SNAP-94847(N-(3-[1-([4-(3,4-difluorophenoxy)phenyl]methyl)(4-piperidyl)]-4methylphenyl)-2-methylpropanamide, $10 \mathrm{mg} / \mathrm{kg}$ for 21 days, IP) to DIO mice resulted in a transient reduction in food intake, adipocyte size and fat mass (Verty et al., 2013). SNAP-94847 decreased immobility time (depressive-like effect) in the Porsolt's forced swim test and normalized immobility time produced by rimonabant. Thus, $\mathrm{MCH} 1$ receptors may have a role in rescuing adverse behavioral effects of rimonabant. A combination pharmacotherapy targeting both $\mathrm{CB} 1$ and $\mathrm{MCH} 1$ receptors may be beneficial for the treatment of obesity. Pharmacological agents that inhibit CB1 receptors or that increase monoacylglycerol lipase and fatty acid amide hydrolase activity (2-AG and anandamide degrading enzyme, respectively) may treat obesity.

Despite similar caloric intake upon exposure to a high-fat diet, CB1 knockout mice are resistant to DIO, suggesting a role for endocannabinoid signaling in the regulation of peripheral energy metabolism (Ravinet Trillou et al., 2004). The role of endocannabinoids in mediating hepatic lipogenesis is indicated by a marked increase in hepatic and hypothalamic mRNA levels of the lipogenic transcription factor, SREBP-1c, and fatty acid synthase following administration of CB1 agonist, HU210 ((6aR,10aR)-9-(hydroxymethyl)-6,6dimethyl-3-(2-methyloctan-2-yl)-6a,7,10,10a-tetrahydrobenzo[c]-chromen-1-ol; $20 \mu \mathrm{g} / \mathrm{kg}$, IP) to mice (Osei-Hyiaman et al., 2005b). Pretreatment with rimonabant (3 mg/kg, IP) blocked these HU210-induced effects.

Endocannabinoid mediation of hepatic lipogenesis is supported also by increased basal rates of de novo fatty acid synthesis in wild-type mice fed a high-fat diet for $3 \mathrm{wk}$, compared to lean controls fed a regular diet (Shimano et al., 1996; Osei-Hyiaman et al., 2005b). Rimonabant ( $3 \mathrm{mg} / \mathrm{kg}$, IP) decreased de novo fatty acid synthesis rate in mice fed high-fat diet, compared to control mice fed high-fat diet. Consistent with these findings, CB1 knockout mice fed a high-fat diet did not exhibit a change in basal rate of fatty acid synthesis, compared to chow-fed controls. Thus, CB1 stimulation increases de novo fatty acid synthesis, contributing to development of DIO. This interpretation is supported by an increase in hepatic levels of anandamide as well as CB1-mediated fatty acid synthesis in mice fed high-fat diet (Osei-Hyiaman et al., 2005b). Thus, the fatty acid biosynthetic pathway represents a common molecular target for central appetitive and peripheral metabolic effects of endocannabinoids.

Obesity is associated with fatty liver, insulin and leptin resistance, and changes in plasma lipid profile. The role of hepatic CB1 receptors in the metabolic consequences of a high-fat diet was studied using liver-specific CB1 knockout mice fed high-fat diet for 14 wk (OseiHyiaman et al., 2008). Compared to wild-type mice, liver-specific CB1 knockout mice fed high-fat diet exhibited decreased steatosis, hyperglycemia, dyslipidemia, and insulin and 
leptin resistance. CB1 agonist (HU210, $20 \mu \mathrm{g} / \mathrm{kg}$, IP)-induced increase de novo hepatic lipogenesis, decrease in carnitine palmitoyl transferase-1 activity and decrease in total energy expenditure were found in CB1 global knockout mice and liver-specific CB1 knockout mice (Osei-Hyiaman et al., 2008). Thus, endocannabinoid activation of hepatic CB1 receptors contributes to steatosis and hormonal and metabolic changes occuring with high-fat diets. However, since liver specific CB1 knockout mice exhibited increases in body weight upon long-term exposure to high-fat diet (Osei-Hyiaman et al., 2008), endocannabinoid activation of hepatic $\mathrm{CB} 1$ receptors does not contribute to the increase in adiposity with high-fat diet. Thus, endocannabinoids act at central CB1 receptors to indirectly influence peripheral energy metabolism, or endocannabinoids produce effects via CB1 receptors at extrahepatic sites (e.g., adipose tissue) (Tedesco et al., 2008). Peripheral $\mathrm{CB} 1$ receptors could be targeted selectively for the treatment of fatty liver, impaired glucose homeostasis and dyslipidemia to minimize neuropsychiatric side-effects of central CB1 receptor antagonism.

Orosensory positive feedback plays a key role in promoting excessive intake of foods rich in dietary fat (Greenberg and Smith, 1996). In addition to regulation centrally, endocannabinoids localized to gut are implicated in positive feedback control of fat preference and intake (DiPatrizio et al., 2011, 2013; DiPatrizio and Piomelli, 2015). To isolate effects of orosensory signals from post-ingestive influences, a sham-feeding paradigm in rats was employed, such that ingested liquid diets are removed from the stomach via a chronically implanted gastric cannula (Schwartz et al., 2008). Sham-feeding of a high-fat liquid meal resulted in accumulation of 2-AG and anandamide in the jejunal small intestine, but not in other peripheral tissues or brain (DiPatrizio et al., 2011). Sham feeding of meals containing either carbohydrate or protein failed to exert this effect, indicating that orosensory properties of fat alone mobilize small intestine endocannabinoids. Vagus nerve transection blocked effects of fat sham feeding on endocannabinoid mobilization, indicating that gustatory signals are transmitted from brainstem to intestine through the vagus nerve. Duodenal infusion of rimonabant $(0.3$ and $1 \mathrm{mg} / \mathrm{kg})$, and systemic administration of peripherally restricted CB1 receptor antagonist, URB447 ([4-amino-1-[(4chlorophenyl)methyl]-2-methyl-5-phenyl-1H-pyrrol-3-yl]phenyl-methanone, 20 mg/kg, IP), reduced sham fat feeding, indicating that gut endocannabinoids may be critical for positive orosensory feedback mechanism driving fat intake (DiPatrizio et al., 2011).

Dietary fats are comprised of complex lipids, such as triglycerides and phospholipids. Gut endocannabinoid signaling may be mediated by textural properties or fatty acid composition of complex lipids. Mineral oil has a texture similar to nutritive oils, but contains no fatty acids (Mindell et al., 1990). Rats were allowed to sham feed either a corn oil emulsion or a liquid diet containingd only mineral oil (DiPatrizio et al., 2013). In contrast to corn-oil emulsion, sham intake of mineral oil did not modify jejunal 2-AG levels, suggesting that dietary fat texture alone is not sufficient to trigger jejunal 2-AG mobilization. Sham-feeding emulsions containing monoenoic or dienoic fatty acids resulted in a 2-fold accumulation of jejunal endocannabinoids, whereas emulsions containing stearic acid or linoleic acid had no effect. Given that peripheral blockade of CB1 receptors reduces intake of corn oil (DiPatrizio et al., 2011), preference for linoleic acid, the most abundant fatty acid constituent of corn oil (Moreau, 2011), over mineral oil was assessed in a 2-bottle choice test in sham 
feeding rats (DiPatrizio et al., 2013). Vehicle-treated rats preferred linoleic acid over mineral oil; this preference was blocked by URB447 (20 mg/kg, IP). Thus, small-intestinal endocannabinoids mediate orexigenic responses produced by tasting fat-containing foods, and gustatory signals elicited by dietary fat-containing selective long chain unsaturated fatty acids are transmitted from brainstem to intestine through the vagus nerve (DiPatrizio et al., 2011, 2013).

Fasting increases anandamide and 2-AG levels in rat duodenum, suggesting that endocannabinoid signaling in small intestine promotes food intake (Gomez et al., 2002; Izzo et al., 2009). Food deprivation (12 and $24 \mathrm{~h}$ ) increases 2-AG and its lipid precursor 1, 2diacylglycerol in rat jejunum mucosa, and re-feeding normalizes this response (DiPatrizio et al., 2015). These findings suggest that gut 2-AG may serve as a hunger signal. Feedingdependent regulation of 2-AG was absent in other peripheral tissues. Increases in jejunal 2AG levels induced by food-deprivation were abrogated by vagus nerve surgical resection or pharmacological blockade of muscarinic M3 receptors using DAU5884 ((8-methyl-8azabicyclo-[3.2.1]octan-3-yl) 2-oxo-1,4-dihydroquinazoline-3-carboxylate, $300 \mathrm{nmol}$ ) in small intestine, but not by inhibition of muscarinic M1 receptors (pirenzepine, $300 \mathrm{nmol}$ ) (DiPatrizio et al., 2015). These results suggest that food deprivation stimulates 2-AGdependent CB1 receptor activation through a mechanism that requires efferent vagal activation of jejunum muscarinic M3 receptors, which promotes feeding after a fast (DiPatrizio et al., 2015). Thus, endocannabinoid overactivity in proximal small intestine may contribute to obesity. Targeted inhibition of endocannabinoid signaling in intestine may control excessive fat intake and reduce obesity. Taken together, both central and peripheral CB1 receptors serve as potential therapeutic targets for the treatment of obesity.

\subsection{Peripherally-derived satiety, hunger and adiposity signals}

Peripherally-derived satiety, hunger and adiposity signals are integrated within the hypothalamic and brainstem circuits to regulate feeding and metabolism. These peripheral signals include short-term, meal-related afferent satiety and hunger signals from gut and long-term afferent signals from adipocytes and pancreatic $\beta$-cells. Peripheral signals that regulate satiety include cholecystokinin (CCK), glucagon-like peptide-1 (GLP-1), peptide YY (PYY), amylin and oleoylethanolamide (OEA). In contrast to satiety signals that promote meal termination, ghrelin, a peptide hormone released by the stomach, promotes meal initiation. Lastly, adiposity signals, including leptin and adiponectin released from white adipocytes and insulin released from pancreatic $\beta$-cells, are involved in body weight regulation. Previous reviews have described peripheral mechanisms that regulate energy homeostasis (Sam et al., 2012; Camilleri, 2015). The current section provides a brief overview of these mechanisms and identifies potential therapeutic targets for obesity.

2.3.1. $C C K$-The peptide hormone, CCK is released from I-cells of the proximal intestine following absorption of fat or protein (Liddle et al., 1985). Molecular forms of CCK range from 4 to 83 amino acids as a result of post-translational processing of products from the pro-CCK gene (Rehfeld et al., 2001). In the human intestine and circulation, predominant CCK forms include CCK-8, CCK-22, CCK-33 and CCK-58. CCK-induced satiety signaling is mediated via CCK receptors, which are members of the GPCR family (Ballinger et al., 
1995; Blevins et al., 2000; Degen et al., 2001). Two CCK receptor subtypes include CCK-A receptors that are expressed in the pancreas, on vagal afferent enteric neurons, NTS, area postrema and dorsomedial hypothalamus and CCK-B receptors that are expressed in stomach, afferent vagus nerve and throughout brain (Dufresne et al., 2006). Inhibition of CCK-A, but not CCK-B receptors, attenuates CCK-induced satiety (Moran et al., 1992). CCK $(100 \mu \mathrm{g} / \mathrm{kg}$, IP)-induced inhibition of glucose consumption in rats was decreased in a dose-dependent manner by CCK-A antagonist, devazepide (L-364718: N-(1-methyl-2oxo-5-phenyl-3H-1,4-benzodiazepin-3-yl)-1H-indole-2-carboxamide, 10-100 $\mu \mathrm{g} / \mathrm{kg}$, IP). On the other hand, the CCK-B antagonist, L-365260 (1-(1-methyl-2-oxo-5-phenyl-3H-1,4benzodiazepin-3-yl)-3-(3-methylphenyl)urea, 10-1000 $\mu \mathrm{g} / \mathrm{kg}$, IP) failed to attenuate the inhibitory effect of CCK on gucose consumption. In agreement with these findings, Otsuka Long Evans Tokushima Fatty (OLETF) rats that lack CCK-A receptors are hyperphagic, obese and diabetic (Bi and Moran, 2002). Evaluation of patterns of hypothalamic gene expression in OLETF rats reveals upregulation of NPY in the dorsomedial hypothalamus, which also may underlie the hyperphagia and development of obesity (Bi et al., 2001).

Investigation of the effects of a stable CCK-8 analog, (pGlu-Gln)-CCK-8, on metabolic control and hypothalamic gene expression in mice fed a high-fat diet for $20 \mathrm{wk}$ provides additional evidence that CCK regulates food intake (Montgomery et al., 2013). (pGlu-Gln)CCK-8 (25 nmol/kg twice daily for 16 days, IP) decreased energy intake, circulating glucose, insulin levels and body weight in mice fed a high-fat diet, compared to lean control mice. However, the CCK analog elevated hypothalamic mRNA levels of NPY and decreased POMC mRNA levels. While these effects suggest an orexigenic effect, it is possible that the reported alterations in mRNA levels represent a counter-regulatory physiological response induced by CCK- 8 analog-induced decreases in feeding. Further elucidation of hypothalamic mechanisms underlying the anorexigenic effects of the CCK-8 analog is needed. Overall, CCK agonists appear to have a role in the regulation of energy balance.

2.3.2. GLP-1-The peptide hormone GLP-1 is synthesized by L-cells of the distal small intestine and is released in response to carbohydrate and fat digestion (Lavin et al., 1998; Thomsen et al., 1999). Two forms of GLP-1 are in blood including the predominant variants GLP-1(7-36) amide and GLP-1(7-37) amide (Marks et al., 1991). GLP-1 mediates appetite control via insulin release, glucagon inhibition as well as absorption and metabolism of macronutrients (Naslund et al., 1999; Flint et al., 2001). GLP-1 receptors are members of the GPCR family and are expressed widely in pancreatic islets, kidney, lung, heart, and numerous regions of the peripheral and central nervous systems (Bullock et al., 1996; Gutzwiller et al., 2004; Drucker, 2006). Within the islets, GLP-1 receptors are localized predominantly to $\beta$-cells (Drucker, 2006).

GLP-1(7-36) or GLP-1 receptor agonists (e.g., exendin-4 or liraglutide) given systemically reduce food intake in a dose-dependent manner in rodents and non-human primates. GLP-1 $(0.5-170 \mathrm{pmol} / \mathrm{kg} / \mathrm{min}$ for $3 \mathrm{~h}, \mathrm{IV})$ at dark onset dose-dependently inhibited food intake in rats (Chelikani et al., 2005). In adult male rhesus macaques trained to lever press for food pellets, a long-lasting GLP-1 receptor agonist (exendin-4, 0.1-3.0 $\mu \mathrm{g} / \mathrm{kg}$, IM) dosedependently suppressed food intake specifically through a reduction in meal size, compared to vehicle-control (Scott and Moran, 2007). In rats, the GLP-1 receptor antagonist, exendin- 
(9-39) (100 $\mu \mathrm{g} / 2 \mu \mathrm{l}, \mathrm{ICV})$, attenuated the decrease in food intake induced by GLP-1 agonists, exendin-4 (3 $\mu \mathrm{g} / \mathrm{kg}$, IP) and liraglutide (10 $\mu \mathrm{g} / \mathrm{kg}$, IP) (Kanoski et al., 2011). Further, to assess the contribution of GLP-1 receptors expressed on subdiaphragmatic vagal afferents to the anorectic effects of exendin- 4 and liraglutide, food intake in rats with complete subdiaphragmatic vagal deafferentation was compared to surgical controls. Both exendin-4 (1 or $3 \mu \mathrm{g} / \mathrm{kg}$, IP) and liraglutide $(10,25$ or $50 \mu \mathrm{g} / \mathrm{kg}$, IP) decreased food intake in controls. However, in rats that underwent subdiaphragmatic vagal deafferentation, only the higher doses of the GLP-1 receptor agonists decreased food intake. These findings suggest that exendin- 4 and liraglutide reductions in food intake are mediated by a combined action on vagal afferents and central GLP-1 receptors. Overall, GLP-1 receptor agonists appear to be a promising target for the treatment of obesity.

2.3.3. $P Y Y$-The 36 -amino acid peptide hormone, $P Y Y$, is released from L-cells of the distal small intestine in response to fatty acids, dietary fibers and bile (Onaga et al., 2002). In human plasma, $\mathrm{PYY}_{1-36}$ predominates over $\mathrm{PYY}_{3-36}$ under fasting conditions; whereas after a meal, $\mathrm{PYY}_{3-36}$ is the major circulating peptide (Grandt et al., 1994a, b). PYY receptors are widely expressed in the central nervous system. PYY 1-36 $_{\text {activates at least three receptor }}$ subtypes including, Y1, Y2, and Y5; $\mathrm{PYY}_{3-36}$ is more selective for the Y2 subtype (Dumont et al., 1995). Direct injection of $\mathrm{PYY}_{3-36}$ into arcuate nucleus as well as peripheral administration of $\mathrm{PYY}_{3-36}$ to rodents increased arcuate c-fos immunoreactivity and decreased food intake (Batterham and Bloom, 2003). While peripheral administration of $\mathrm{PYY}_{3-36}$ inhibited food intake in wild-type control mice, $\mathrm{PYY}_{3-36}$ did not alter food intake in mice with an arcuate-specific deletion of Y2 receptors (Sainsbury et al., 2002; Batterham and Bloom, 2003). These results indicate that the anorectic effects of $\mathrm{PYY}_{3-36}$ are mediated via Y2 receptors.

Expression of a genetic variant of the PYY gene, PYY Q62P, correlated with body weight in obese compared to lean men, suggesting that sequence variants within the PYY gene may influence susceptibility to obesity (Ahituv et al., 2006). Compared to control, obese humans exhibited decreased plasma PYY levels as well as an attenuated PYY response to meals across a range of caloric content (le Roux et al., 2006). Importantly, in obese humans, high caloric content was required to increase plasma PYY relative to normal-weight humans (le Roux et al., 2006). Co-administration of PYY with GLP-1 produced a synergistic effect by decreasing energy intake and promoting satiety in healthy subjects, suggesting benefit of a combination therapy (Steinert et al., 2010). Thus, reduced plasma PYY appears to underlie decreased satiety and increased food intake in obesity.

2.3.4. Amylin-Amylin, a 37 -amino acid peptide hormone released with insulin from pancreatic $\beta$-cells in response to high glucose levels in blood, reduces both food intake and body weight though actions in brain stem (Rushing et al., 2001). Amylin (0.1-1 $\mu \mathrm{g} / \mathrm{kg}$, IP) dose-dependently reduced food intake in rats (Lutz et al., 1994). Anorectic effects of amylin are mediated by amylin receptor complexes in brain stem composed of calcitonin receptors coupled to receptor activity-modifying proteins (Chen et al., 1997; Christopoulos et al., 1999). A synthetic analog of amylin, pramlintide, is available for the treatment of both type 1- and type 2-diabetes (Whitehouse et al., 2002; Hollander et al., 2003). Diabetic patients 
treated with pramlintide for a year exhibited weight loss relative to placebo-treated controls (Hollander et al., 2003). Leptin is an adipose-derived hormone that decreases food intake and increases lipolysis (Halaas et al., 1995; Campfield et al., 1996). Concurrent peripheral administration of amylin and leptin resulted in synergistic weight loss in leptin-resistant DIO rats, and amylin pre-treatment restored leptin signaling, suggesting that amylin restores leptin sensitivity in the DIO model (Roth et al., 2008). A pharmacologically optimized amylin analog, davalintide, has undergone Phase II clinical trials for the treatment of obesity (Colon-Gonzalez et al., 2013). Further clinical development of davalintide has not been reported. Overall, amylin analogs as well as a combination of amylin and leptin exhibit therapeutic potential for the treatment of obesity.

2.3.5. OEA-Food intake stimulates small-intestinal mucosal cells to produce the lipid messenger, OEA, which serves as a satiety signal (Rodriguez de Fonseca et al., 2001; Fu et al., 2007; Piomelli, 2013). Investigation of the nutrients involved in OEA generation revealed that intraduodenal infusion of fat stimulates intestinal OEA production in rats, whereas, infusion of proteins and carbohydrate did not produce this effect (Schwartz et al., 2008). Thus, dietary fat is necessary and sufficient to trigger the production of OEA by small intestinal enterocytes. Both peripheral $(5-20 \mathrm{mg} / \mathrm{kg}$, IP) and oral $(50-200 \mathrm{mg} / \mathrm{kg})$ administration of OEA decreases in a dose-dependent manner food intake in rodents (Gaetani et al., 2003; Oveisi et al., 2004). Moreover, sub-chronic administration of OEA (5 $\mathrm{mg} / \mathrm{kg}$ once/day for $2 \mathrm{wk}, \mathrm{IP}$ ) reduces food intake, lowers body weight and decreases serum cholesterol and triglyceride levels in obese Zucker rats, compared to vehicle-treated control (Fu et al., 2005). These results suggest that in addition to satiety, OEA regulates lipid metabolism.

OEA elicits satiety primarily by prolonging the post-meal interval and reducing meal frequency (Gaetani et al., 2003; Oveisi et al., 2004). OEA-induced anorexic response involves $i$ ) local activation of the peroxisome proliferator-activated receptor-a, which is implicated in regulating absorption, storage and utilization of dietary fat; ii) stimulation of afferent vagus nerve fibers; and iii) recruitment of appetite-controlling paraventricular nucleus and NTS circuits (Rodriguez de Fonseca et al., 2001; Fu et al., 2003; Evans et al., 2004; Bookout et al., 2006; Lefebvre et al., 2006; Gaetani et al., 2010; Azari et al., 2014; Provensi et al., 2014). Hypophagic effects of exogenous OEA are abolished by genetic deletion of peroxisome proliferator-activated receptor- $a$ and are mimicked closely by administration of synthetic peroxisome proliferator-activated receptor-a agonists (Astarita et al., 2006; Fu et al., 2003). Anorexic effects of OEA were absent in rats treated with capsaicin to remove the peripheral sensory fibers, supporting a role for afferent vagus fibers in mediating hypophagic effects of OEA (Rodriguez de Fonseca et al., 2001). In contrast, subdiaphragmatic vagotomy in rats did not block OEA-induced hypophagia, necessitating further investigation of the role of vagal afferents in OEA-mediated satiety signaling (Azari et al., 2014; DiPatrizio and Piomelli, 2015). Food deprivation decreased OEA mobilization in jejunum of lean rats, while feeding restores jejunal OEA levels (Rodriguez et al., 2001; Fu et al., 2007). Feeding-dependent OEA production is disrupted in gut of DIO rodents, suggesting that high-fat DIO is accompanied by alterations in post-digestive machinery responsible for OEA biosynthesis, which may contribute to reduced satiety and hyperphagia 
(Igarashi et al., 2015). Mechanistic understanding of intestinal OEA signaling in obesity will assist in the development of pharmacological strategies to control appetite in obesity.

2.3.6. Ghrelin-Ghrelin, a 28 -amino acid appetite-stimulating peptide, is found in highest concentrations in gut (Korbonits and Grossman, 2004). In addition to peripheral localization, ghrelin is synthesized in the arcuate (Mondal et al., 2005). Ghrelin crosses the blood brain barrier and binds to ghrelin receptors, a growth hormone secretagogue receptor $1 \mathrm{~A}$ (GHSR1A), expressed in hypothalamus (Cummings, 2006; Harrold et al., 2008). Ghrelin levels are dependent on nutritional status such that peak levels occur prior to meal initiation, followed by a decrease in levels upon food consumption (Cummings et al., 2001; Callahan et al., 2004). Compared to lean controls, obese individuals are more sensitive to appetitestimulating effects of ghrelin (Druce et al., 2005).

Ghrelin regulates adipocyte metabolism through a central mechanism. Compared to vehicle control, ghrelin ( $2.5 \mathrm{nmol} / \mathrm{day}$ for 6 days, ICV) decreased expression of thermogenesisrelated mitochondrial uncoupling proteins 1 and 3 in brown adipocytes; while in white adipocytes, gherlin increased mRNA expression of fat storage-promoting enzymes (lipoprotein lipase, acetyl-CoA carboxylase $a$, fatty acid synthase and stearoyl-CoA desaturase-1) and decreased mRNA of carnitine palmitoyl transferase-1a, the rate-limiting enzyme in fat oxidation (Theander-Carillo et al., 2006). Expression of these fat storage enzymes was decreased in ghrelin-deficient mice, compared to wild-type control (TheanderCarillo et al., 2006). When exposed to a high-fat diet, both GHSR1A knockout mice and ghrelin-deficient mice exhibit resistance to DIO, compared to respective wild-type controls (Cummings, 2006). Vaccination against ghrelin decreases body weight gain in adult male Wistar rats (Zorrilla et al., 2006). These findings suggest that ghrelin receptor antagonists have therapeutic potential. Further investigation of ghrelin antagonists in animal models of obesity and in obese humans is needed.

2.3.7. Leptin—Leptin, a leptin gene (Lep(ob)) product, is produced and secreted by white adipocytes in proportion to fat mass (Zhang et al., 1994; Maffei et al., 1995; Porte et al., 2002). Leptin decreases food intake and body weight by activating leptin receptors in the arcuate (Fei et al., 1997; Elmquist et al., 1998; Myers et al., 2008). Both leptin deficiency (ob/ob mice) and leptin resistance ( $\mathrm{db} / \mathrm{db}$ mice; defective leptin receptors) result in hyperphagia and decreased energy expenditure (Tartaglia et al., 1995; Lee et al., 1996). Importantly, correction of the leptin deficiency in ob/ob mice results in a reduction in food intake and body weight (Halaas et al., 1995; Campfield et al., 1996). Leptin production increases proportionally with adiposity, such that leptin levels are increased in rodent and human models of DIO (Halaas et al., 1995; Heymsfield et al., 1999; Levin and DunnMeynell, 2002). However, increased leptin levels in obesity leads to decreased central leptin sensitivity and increased leptin receptor resistance, and as a result, obese individuals fail to respond to leptin treatment (Levin and Dunn-Meynell, 2002). Nevertheless, administration of both leptin and amylin result in a synergistic decrease in food intake and body weight in rats (Roth et al., 2008). Importantly, amylin restored hypothalamic leptin receptor signaling in obesity (Roth et al., 2008). Also, CCK potentiates leptin effects to decrease food intake 
and body weight (Matson and Ritter, 1999; Colon Gonzalez et al., 2013), suggesting the potential combination of CCK and leptin for the treatment of obesity.

2.3.8. Insulin-Insulin is a metabolic polypeptide hormone synthesized in the pancreas by the $\beta$-cells of the islets of Langerhans (Woods et al., 1974; Brange and Langkjoer, 1993). Similar to leptin, plasma insulin levels correlate positively with adiposity (Woods et al., 1974). Insulin-induced decreases in food intake and body weight are mediated via stimulation of hypothalamic insulin receptors (Menendez and Atrens, 1991). Central administration of an antisense oligodeoxynucleotide designed to blunt the expression of hypothalamic insulin receptors resulted in hyperphagia and increased fat mass in rats (Obici et al., 2002). Compared to control non-diabetic rats, streptozotocin-treated diabetic rats exhibited increased NPY expression in the paraventricular nucleus, ventral medial hypothalamus and lateral hypothalamus; and, insulin replacement normalized NPY levels in these diabetic rats (Williams et al., 1989). With respect to interactions between insulin and melanocortin systems in hypothalamus (Benoit et al., 2002), immunohistochemical analysis revealed insulin receptor expression on arcuate POMC neurons. Also, administration of insulin $(4 \mathrm{mU} / \mu \mathrm{l}, \mathrm{ICV})$ to fasted rats increased POMC mRNA expression. Further, the anorectic actions of insulin were blocked by non-selective melanocortin antagonist, SHU-9119 (Ac-Nle-c[Asp-His-dNal(2')-Arg-Trp-Lys]-NH $2,0.1 \mathrm{nmol} / \mu \mathrm{l}, \mathrm{ICV})$. These results suggest a role for the hypothalamic melanocortin system in mediating the anorexic effects of central insulin. Thus, both melanocortin and NPY systems appear to be important downstream mediators of the anorectic effects of insulin.

2.3.9. Adiponectin-Adiponectin, a 244 -amino acid polypeptide, is another adipocytederived protein that decreases body weight and plasma lipid levels, and enhances the ability of insulin to suppress hepatic glucose production (Berg et al., 2001; Qi et al., 2004; Kadowaki and Yamauchi, 2005). Plasma adiponectin levels increase following food restriction in rodents and following weight loss induced by a calorie-restricted diet or gastric partition surgery in obese humans (Hotta et al., 2000; Berg et al., 2001; Yang et al., 2001). Peripheral administration of adiponectin to rodents attenuates body weight gain without altering food intake (Berg et al., 2001; Fruebis et al., 2001). Adiponectin effects on energy expenditure in rats appear to be mediated within the hypothalamus, as indicated by increased c-fos expression in the paraventricular nucleus (Qi et al., 2004). In contrast to carbohydraterich foods, diets including soy protein, fish oils and linoleic acid increase plasma adiponectin levels (Pischon et al., 2005). Two distinct adiponectin receptors, adipoR1 and adipoR2, are expressed highly in skeletal muscle and liver, respectively, and in hypothalamus (Yamauchi et al., 2003; Qi et al., 2004). Expression of adipoR1 and adipoR2 are decreased in muscle and adipose tissue of ob/ob mice, suggesting decreased adiponectin sensitivity (Tsuchida et al., 2004). In obese humans, an inverse correlation between plasma adiponectin and insulin resistance was found (Arita et al., 1999; Matsuzawa, 2010). Therapeutic strategies that increase adiponectin signaling may be beneficial to ameliorate obesity and insulin resistance.

In summary, regulation of appetite and body weight involves a complex and interacting network of neural systems and peripherally-derived signals. Importantly, these central and 
peripheral mechanisms present numerous viable targets for pharmacotherapeutic intervention.

\section{Non-homeostatic hedonic regulation of food intake}

Brain reward systems play a major role in feeding behavior (Saper et al., 2002; Lutter and Nestler, 2009). Hedonic properties of food can stimulate non-homeostatic feeding, despite that energy requirements have been met, contributing to weight gain and obesity (Berthoud, 2011). Palatable sugar- and fat-rich foods are salient, motivating, natural rewards that can override homeostatic signals (Berthoud, 2011). Palatable food consumption is associated with reward processing of food-related sensory cues regulating preference and hedonic value (Rolls, 2011). Orosensory properties (taste, texture and smell), and post-ingestive effects of palatable foods drive preference and promote excessive food consumption, relative to less palatable foods (Warwick and Weingarten, 1995; Sclafani et al., 1998; Macht and Mueller, 2007).

Hedonic properties of palatable foods and addictive drugs are regulated in brain by a common reward circuitry, such that excessive consumption of palatable foods and drugs produces similar neuroadaptation (Volkow et al., 2008, 2012; Kenny, 2011). Both nonhomeostatic food consumption and drug self-administration activate mesolimbic dopamine (DA) pathways (Koob and Volkow, 2010). While drugs typically activate the reward circuitry via direct effects, food activates this circuitry through cephalic or post-ingestive mechanisms that indirectly activate mesolimbic DA systems. Obesity and substance use disorders are defined by i) abnormal enhancement of reinforcer saliency, ii) excessive consumption habits that strengthen with behavioral repetition, and iii) difficult to control behaviors, despite potential untoward consequences (Berridge and Robinson, 1998; Volkow et al., 2012). Neurobiological mechanisms of drug abuse provide a framework for understanding non-homeostatic hedonic mechanisms and motivational processes underlying palatable food consumption in obesity.

Functional magnetic resonance imaging (fMRI) evaluating blood-oxygen level dependent (BOLD) activity in brain reveals that hedonic food consumption and food-related sensory cues activate mesocorticolimbic reward circuits including orbitofrontal cortex, insula, striatum, amygdala, lateral hypothalamus, VTA and substantia nigra (Pelchat et al., 2004; Simmons et al., 2005; Schur et al., 2009; Bragulat et al., 2010). Immediate early geneexpression (c-fos) in rats exposed to palatable foods shows increased activation of of striatum, nucleus accumbens, amygdala and other limbic structures (Angeles-Castellanos et al., 2007). Lateral habenula deep brain stimulation decreased non-homeostatic and hedonic sucrose self-administration in rats (Friedman et al., 2011). Lesion of lateral habenula delayed extinction responding and increased sucrose-seeking behavior. Results such as these suggest that non-homeostatic, hedonic feeding behavior is regulated by brain regions implicated in reward processing and motivation.

Regarding response to food-related sensory cues, exposure of healthy non-obese women to visual cues (palatable food photographs) resulted in greater BOLD signals and activation in dorsolateral prefrontal cortex, lateral orbitofrontal cortex, insular cortex, dorsal striatum, 
accumbens, amygdala, hypothalamus and brainstem, compared with non-food object photos (Schur et al., 2009). Compared with non-appetitive odors, palatable food-related odors elicited greater BOLD signals in medial prefrontal cortex, accumbens and VTA, when response was collapsed across obese and control; obese women exhibited greater BOLD activation in hippocampus, compared to lean women (Bragulat et al., 2010). Upon tasting palatable foods, both obese men and women exhibited enhanced somatosensory cortex activity, and greater activation of insula and midbrain, compared to lean individuals (Wang et al., 2002). Thus, brain reward relevant regions are activated in response to palatable foodrelated visual, olfactory and taste cues, and obesity may be associated with increased sensitivity to these sensory cues.

In obese humans, striatal DA D2 receptor density is decreased in comparison to non-obese individuals (Wang et al., 2001; Stice et al., 2008). Decreased D2 density may be compensatory for reduced reward circuit activation. Striatal D2 receptor availability in obese humans was decreased relative to control and was positively correlated with glucose metabolism in dorsolateral prefrontal, medial orbitofrontal, anterior cingulate gyrus and somatosensory cortices (Volkow et al., 2009), suggesting that decreased striatal D2 receptors contribute to overeating via modulation of striatal prefrontal pathways, which participate in inhibitory control and salience attribution. Whether decreases in striatal D2 receptor density precede development of obesity or are a consequence of obesity remains unclear. Increases in extracellular DA in dorsal striatum, but not ventral striatum, were found in response to nonhedonic food stimulation (food display without consumption) in food-deprived, normal weight men and women (Volkow et al., 2002). DA was correlated with increased hunger and food desire. Thus, striatal DA signaling plays a distinct role in food motivation, different from nucleus accumbens DA in regulating food reward.

An obesity model posits that non-homeostatic hedonic food intake reflects an imbalance between circuits that motivate behavior (due to involvement in reward and conditioning) and circuits that control and inhibit pre-potent responses (Volkow et al., 2008). This model identifies circuits for $i$ ) reward-saliency, ii) motivation-drive, iii) learning-conditioning and iv) inhibitory control-emotional regulation-executive function. In vulnerable individuals, consumption of large quantities of palatable food disrupts the balance, resulting in enhanced sensitivity of the reward circuitry to conditioned stimuli associated with palatable foods and impaired function of executive control circuitry weakening inhibitory control over appetitive behaviors. DA-, opioid- and endocannabinoid mediated regulation of reward circuits in the context of nonhomeostatic hedonic food intake and the development of obesity is discussed herein.

\subsection{DA}

DA regulates appetitive and food-motivated behavior and facilitates conditioning to food cues (Mark et al., 1994; Martel and Fantino, 1996; Wang et al., 2002). Palatable foods ingestion results in accumbal and dorsal striatal DA release, mediating primary reward, motivation and habit formation (Martel and Fantino, 1996; Small et al., 2003; Kelley, 2004; Yin et al., 2004). Neural systems regulating reward and motivation underlie behaviors associated with compulsive food intake (Volkow and Wise, 2005; Johnson and Kenny, 
2010). Compulsive, non-homeostatic consumption of large quantities of highly palatable food in a short time period characterizes binge eating in humans (American Psychiatric Association, 2013).

Dysregulated striatal DA function is a potential mechanism underlying excessive food intake in obesity. Consistent with human studies, striatal D2 receptors are downregulated in rodents fed a palatable high-fat diet for an extended time (Colantuoni et al., 2001; Johnson and Kenny, 2010; Narayanaswami et al., 2013). Striatal D2 receptor knockdown, using a lentiviral vector delivering a short hairpin interfering RNA (lenti-D2Rsh), increases brain reward threshold and accelerates reward dysfunction and compulsive eating upon extended access to a palatable high-fat diet, compared to lenti-control lean rodents (Johnson and Kenny, 2010). In contrast, reward thresholds are not altered in lenti-D2Rsh and lenti-control rats with extended chow access. Thus, extended access to palatable foods elevates brain reward thresholds, compared to both chow access and restricted access to palatable foods. Lenti-D2Rsh rats with extended access to palatable foods showed increased resistance to disruption of food consumption by aversive conditioned stimuli, relative to lenti-control lean rats. Compulsive-like feeding behavior is measured as palatable food consumption that is resistant to disruption by an aversive conditioned stimulus. These results suggest that deficits in neural reward responses and maladaptive behavioral responses in obesity arise from highfat diet-induced deficits in striatal D2 receptor signaling. Excessive consumption of palatable energy-dense food over stimulates brain reward systems and decreases reward system sensitivity leading to compulsive-like food seeking behavior. Decreases in striatal D2 receptors likely precede the development of obesity and the increased vulnerability to maladaptive behavioral responses upon palatable food exposure, which compensates for a reward deficit. Over consumption of palatable foods triggers neuroadaptive reward circuit responses leading to compulsive food-seeking, and deficits in striatal D2 receptor signaling supports this addictive process.

Evidence reveals dysregulation of DA homeostasis in response to prolonged exposure to high-fat diet and/or obesity. Accumbens DA turnover was decreased in obese rats with freeaccess and non-obese rats with restricted access to high-fat diet for $12 \mathrm{wk}$, when compared to free-access to standard chow (Davis et al., 2008). DA release from accumbens shell, striatal and medial prefrontal cortex was decreased in inbred obese rats compared to those inbred for obesity resistance, both maintained on standard chow (Geiger et al., 2008). In cultured VTA neurons from these inbred obesity-prone rats, tyrosine hydroxylase mRNA expression, DA transporters (DAT) and D2 receptors were decreased. Collectively, deficient DA homeostasis contributes to decreased food-associated primary reward and to the obesity phenotype.

DA is regulated primarily by DAT, which translocates DA across the plasmalemma from the extracellular space into the cytosol of DA neurons (Sulzer et al., 2005). As revealed by genetic-linkage analysis, binge eating is associated with DAT gene polymorphisms (greater frequency of short alleles; 7 or 9 repeats) and reduced DAT expression (Heinz et al., 2000; Fuke et al., 2001; Shinohara et al., 2004). DAT-deficient mice have increased extracellular DA and exhibit greater food intake relative to wild-type mice (Pecina et al., 2003). Kinetic analysis and no-net flux microdialysis reveal that striatal DAT function is decreased and 
extracellular DA increased in a DIO model of obesity-prone outbred rats fed high-fat diet for $8 \mathrm{wk}$, compared to obesity-resistant rats fed the same diet (Narayanaswami et al., 2013).

Decreased DAT function and DA dysregulation did not precede DIO development, but were consequences of obesity (Narayanaswami et al., 2013). Decreased DAT function in obesity may be explained by decreased D2 autoreceptor function, given that D2 autoreceptor activation increases DAT function (Cass and Gerhardt, 1994). Quinpirole (3-100 nmol/L) stimulation of D2 receptors attenuated the inhibition of VTA DA neurons in obese mice, compared with lean mice (Koyama et al., 2014). Investigation of D2 agonist-induced facilitation of DAT function in obesity may provide insight about the ability D2 agonists to normalize DA homeostasis and ameliorate maladaptive eating behaviors.

The D2 receptor agonist, bromocriptine, reduced body fat, increased lean muscle mass, increased glucose tolerance and insulin function, and reduced triglycerides and free fatty acids, compared to control (Cincotta and Meier, 1996; Pijl et al., 2000). Bromocriptine has been evaluated in leptin receptor-deficient obese Zucker rats and in polygenetic DIO rat models (Davis et al., 2009). Bromocriptine (10 mg/kg daily for $4 \mathrm{wk}$, IP) increased D2 receptor expression in obese Zucker rats, and increased DAT expression in DIO rats. In obese Zucker rats, D2 receptor density correlated inversely with food intake, and directly with locomotor activity. In DIO rats, DAT expression correlated inversely with food intake, body fat composition and directly with locomotor activity. These results underscore bromocriptine's potential in ameliorating behavioral and pathophysiological components of obesity and suggest that pre- or postsynaptic D2 receptors and/or DAT may serve as targets for the treatment of obesity.

\subsection{Opioids: Role of $\mu$-opioid receptors}

Opioids modulate caloric intake, macronutrient preference and motivational aspects of feeding behavior. Endogenous opioid peptides (endorphins, enkephalins, dynorphins and endomorphins) act at $\mu-, \delta$ - or $\kappa$-opioid receptors, members of the GPCR family (Mansour et al., 1994; Nogueiras et al., 2012). $\mu$-Opioid receptor signaling has a role in the regulation of non-homeostatic hedonic food intake, however, a limited understanding of the role of $\delta$ - or $\kappa$-opioid receptors exists. Rats treated with $\mu$-opioid receptor agonist, morphine (10-30 $\mathrm{mg} / \mathrm{kg}$, IP), exhibit increased fat intake and decreased carbohydrate intake compared to control (Marks-Kaufman and Kanarek, 1980). Naloxone (0.1-10 mg/kg, IP), a non-selective opioid antagonist, decreased fat intake (Marks-Kaufman and Kanarek, 1981). Opioid signaling in accumbens shell modulates food palatability and non-homeostatic hedonic feeding (Peciña and Berridge, 2005). In satiated rats, intra-accumbens infusion of $\mu$-opioid receptor agonist, DAla ${ }^{2}, \mathrm{~N}, \mathrm{Me}-\mathrm{Phe}^{4}, \mathrm{Gly}_{\mathrm{ol}}{ }^{5}$ enkephalin $(0.025-2.5 \mu \mathrm{g} / 0.5 \mu \mathrm{l})$, increased palatable food consumption and preference for high-fat diet vs. high-carbohydrate diet (Zhang et al., 1998). Irreversible $\mu$-opioid receptor antagonist, $\beta$-funaltrexamine $(8 \mathrm{nmol} / 0.8$ $\mu \mathrm{l})$, into accumbens shell selectively decreased palatable food consumption, underscoring the role of $\mu$-opioid receptors in hedonic eating (Ward et al., 2006). Competitive $\mu$-opioid receptor antagonist, 6-(4-\{[(3-methylbutyl)amino]methyl $\}$ phenoxy)-nicotinamide (10 or 50 $\mathrm{mg} / \mathrm{kg}$ and 1,3 or $10 \mathrm{mg} / \mathrm{kg}, \mathrm{PO}$ ), given to DIO mice and rats, respectively, dosedependently decreased acute food intake and weight gain (Zhang et al., 2006). Compared to wild-type mice, $\mu$-opioid receptor deficient mice are resistant to DIO, attributed to increased 
skeletal muscle expression of carnitine palmitoyl transferase-1 and increased fatty acid oxidation (Tabarin et al., 2005). Thus, results support the therapeutic potential of $\mu$-opioid receptor antagonists for obesity.

$\mu$-Opioid signaling in food-motivated behavior has been assessed using a progressive ratio (PR) schedule of reinforcement (Cleary et al., 1996; Zhang et al., 2003; Papaleo et al., 2007). Animals press a lever to obtain food reinforcement, and number of active lever responses required to obtain a reinforcer is increased progressively within a session, i.e., each successive reinforcer requires increased effort (Richardson and Roberts, 1996). Escalation of response requirements continues until responding ceases (breakpoint), indicating the motivation level (Richardson and Roberts, 1996; Shippenberg and Koob, 2002). Naloxone $(0.3-10 \mathrm{mg} / \mathrm{kg}$, IP) dose-dependently decreased the breakpoint to obtain a sucrose reinforcer in rats (Cleary et al., 1996). Infusion of D-Ala ${ }^{2}, \mathrm{~N}, \mathrm{Me}-\mathrm{Phe}^{4}, \mathrm{Gly}_{-} \mathrm{ol}^{5}$ enkephalin $(0.25 \mu \mathrm{g} / 0.5 \mu \mathrm{l})$ into accumbens core increased breakpoint for sucrose (Zhang et al., 2003). Using knockout mice, the specific role of $\mu$-opioid receptors in motivational properties of food intake was assessed (Papaleo et al., 2007). During a fixed ratio-1 schedule ( 1 response provides 1 reinforcer), nose-poke responding was not different between $\mu$-opioid receptor knockout and wild-type mice, suggesting that cognitive and learning processes were not altered. Compared to wild-type, responding for chow or sucrose pellets was decreased in $\mu$-opioid receptor knockout mice during fixed ratio-3 responding (3 responses provide 1 reinforcer). Under PR, $\mu$-opioid receptor knockout mice had lower breakpoints for chow or sucrose pellets compared to wild-type, supporting a role of $\mu$-opioid receptors in mediating food-motivated behavior. $\mu$-Opioid receptor knockouts had higher breakpoints for sucrose pellets than for chow pellets; thus, genetic inactivation of $\mu$-opioid receptors did not eliminate sucrose-directed behavior. Decreased motivation to eat despite preserved hedonic processing of sucrose pellets in $\mu$-opioid receptor knockout mice support the role of $\mu$-opioid receptors in motivational properties of ingestive behavior, which may be independent of hedonic properties of food intake. Together, these findings indicate $\mu$-opioid receptors are potential targets for decreasing food motivated behavior.

Inhibition of $\mu$-opioid receptors prevents both food seeking and binge-like eating in rats, as demonstrated using a second-order schedule of chocolate pellet reinforcement, measuring both motivation for palatable food and impact of ingested food on subsequent food seeking (Everitt et al., 1987; Giuliano et al., 2012). In this complex schedule, every 10 lever presses results in a brief $(1 \mathrm{~s})$ cue light signaling a fixed interval $15 \mathrm{~min}$ schedule. After the $15 \mathrm{~min}$, 10 lever presses results in delivery of chocolate pellets and cue light for $20 \mathrm{~s}$. Number of chocolate pellets delivered at the end of the fixed interval increased progressively from 2 to 20. GSK1521498 ((N-[[2, 6-difluoro-4-[3-(1H-1, 2, 4-triazol-5-yl)phenyl]phenyl]methyl]-2, 3-dihydro-1H-inden-2-amine; $0.1-3 \mathrm{mg} / \mathrm{kg}$, IP) or naltrexone $(0.1-3 \mathrm{mg} / \mathrm{kg}, \mathrm{SC})$ were given 30 and $10 \mathrm{~min}$, respectively, before the session. GSK1521498 has a 14-fold greater selectivity for $\mu$-opioid over $\delta$ - and $\kappa$-receptors (Ignar et al., 2011; Kelly et al., 2015). In the first interval, GSK1521498 ( 1 and $3 \mathrm{mg} / \mathrm{kg}$ ) decreased responding compared to control; however, there was no effect in naltrexone-treated rats. In the post-ingestive second interval, both GSK1521498 ( 1 and $3 \mathrm{mg} / \mathrm{kg}$ ) and naltrexone ( 1 and $3 \mathrm{mg} / \mathrm{kg}$ ) decreased responding. Thus, both GSK1521498 and naltrexone reduced palatable food seeking post ingestion, indicating a reduction in hedonic value. However, only GSK1521498 reduced seeking before 
ingestion, indicating a role of $\mu$-opioid receptors in incentive motivation for palatable food seeking behavior.

Binge eating is characterized by consumption of an unusually large amount of high calorie food (fat and/or sugar) during a discrete time period (American Psychiatric Association, 2013). Binge eating and increased response to food-associated stimuli are associated frequently with obesity. $\mu$-Opioid receptor availability in reward brain areas increases in response to binge eating of palatable foods (Colantuoni et al., 2001). Rats were provided with access to chow with or without $25 \%$ glucose solution for $12 \mathrm{~h}$, followed by a 12 -h period of food deprivation each day. After 10 days, exposure to glucose resulted in doubled glucose intake and excessive chow intake in the first h of daily access. After 30 days, $\mu$ opioid receptor expression was increased in cingulate cortex, hippocampus, locus coeruleus and accumbens shell in the glucose binge group, compared to chow-only controls (Colantuoni et al., 2001).

Binge-like eating of a highly palatable chocolate diet was determined after GSK1521498 (0.1-3 mg/kg, IP) and naltrexone (0.1-3 mg/kg, SC) in rats (Cottone et al., 2008; Giuliano et al., 2012). Brief access (10 min) to chocolate diet promoted rapid consumption of large amounts of chocolate and self-restriction of chow intake in anticipation of palatable food access. Both GSK1521498 and naltrexone dose-dependently reduced binge-like palatable food hyperphagia, indicating that inhibition of $\mu$-opioid receptors reduces motivational properties of stimuli eliciting binge eating and regulates hedonic mechanisms of binge-like eating. Thus, $\mu$-opioid receptor antagonists may assist with treatment of maladaptive eating, common with obesity.

A randomized, double-blind, placebo-controlled, single dose, 2-way crossover design evaluated GSK1521498 (25 mg, PO) effects on hedonic taste preference (pleasantness) and taste perception using 20 samples of sweetened commercial dairy products, with varying sucrose and fat content, and a standard 9-point hedonic preference scale that ranged from dislike extremely to like extremely (Nathan et al., 2012). Compared with placebo, GSK1521498 reduced hedonic ratings for the highest levels of fat and sucrose. GSK1521498 selectively reduced caloric intake of snack foods in the high-fat/high-sugar category, while having no effects on low-fat/low-sugar, lowfat/high-sugar and high-fat/low-sugar categories. These results support the role of $\mu$-opioid receptors in non-homeostatic hedonic aspects of palatable food consumption and identify $\mu$-opioid receptors as potential therapeutic targets for the treatment of maladaptive eating behavior associated with obesity.

\subsection{Endocannabinoids}

In addition to contributing to homeostatic energy balance, endocannabinoids regulate hedonic aspects of food intake. Although pharmacological and genetic studies suggest a role of CB2 receptors in drug addiction (Navarette et al., 2013), this is not as well defined in food reward. CB2 receptors expression in striatum, hippocampus and thalamus (Wotherspoon et al., 2005; Gong et al., 2006; Onaivi et al., 2006) with overlap in reward circuits underlying obesity and addiction (Volkow et al., 2008; Kenny, 2011) suggest CB2 participation in nonhomeostatic, hedonic food intake. Diacylglycerol lipase-a, endocannabinoids, 2-AG, anandamide and CB1 receptors localized to accumbens shell and VTA play a role in 
motivation and reward mechanisms underlying food intake (Di Marzo et al., 2009).

Acquisition of conditioned place preference (CPP) associated with sucrose pellet consumption was decreased dose-dependently by pretreatment with rimonabant (0.03-3 $\mathrm{mg} / \mathrm{kg}$, IP; Chaperon et al., 1998). Motivation for palatable food was assessed using PR schedules in CB1 knockout and wild-type mice (Sanchis-Segura et al., 2004). CB1 knockouts exhibited decreased PR breakpoints for sucrose reinforcement, indicating reduced motivation for palatable food. Thus, CB1 receptors are important targets mediating reinforcing and motivational properties of palatable foods.

Activation of CB1 receptors on GABAergic terminals in VTA disinhibits DA neurons, leading to increased VTA firing (Riegel and Lupica, 2004). Activation of CB1 receptors decreases excitatory glutamatergic input to VTA and nucleus accumbens, thereby regulating activity of neurons projecting from prefrontal cortex (Melis et al., 2004). Endocannabinoids do not directly depolarize DA neurons, but indirectly act via pre- and postsynaptic inhibition of interneurons and as retrograde messengers at CB1 receptors, modulating glutamatergic and GABAergic synaptic inputs in VTA and accumbens (Fride, 2002; Szabo et al., 2002; Piomelli, 2005). Thus, endocannabinoid modulation of DA function depends on GABAergic inhibitory and glutamatergic excitatory balance in VTA (Maldonado et al., 2006).

Endocannabinoid signaling within accumbens shell is implicated in food intake and hedonic impact of palatable foods. 2 -AG $(0.5 \mu \mathrm{g} / 0.5 \mu \mathrm{l})$ or anandamide $(25 \mathrm{ng} / 0.5 \mu \mathrm{l})$ into rat accumbens shell increased food intake (Kirkham et al., 2002; Mahler et al., 2007), which was inhibited by rimonabant ( $0.5 \mathrm{mg} / \mathrm{kg}$; SC; Kirkham et al., 2002). Anandamide enhanced hedonic 'liking' reactions (orofacial expressions) elicited by sucrose taste (Berridge, 2000; Mahler et al., 2007). Compared with chow, highly palatable food (candied cherry) consumption increased DA in rat accumbens shell; rimonabant (0.3 and $1 \mathrm{mg} / \mathrm{kg}$, IP) decreased DA and highly palatable food consumption (Melis et al., 2007). Inhibitory effects of rimonabant on DA were prevented by pretreatment with a CB1 agonist, WIN 55,212-2 $((R)$-(+)-[2,3-dihydro-5-methyl-3[(4-morpholinyl)-methyl]pyrrolo-[1,2,3-de]-1,4benzoxazinyl]-(1-naphthalenyl)-methanone; $0.3 \mathrm{mg} / \mathrm{kg}$, IP). Thus, rimonabant attenuates palatable food-induced increases in accumbal DA, confirming mesolimbic DA involvement in mediating endocannabinoid effects on palatable food intake.

Compared to standard chow, rats fed a palatable, high-fat diet for 8 wk exhibited decreased CB1 receptor expression in cortex, entopeduncular nucleus, accumbens and hippocampus, which was interpreted as adaptive to elevated endocannabinoids following long term exposure to palatable food (Harrold et al., 2002). Exposure to food with high incentive salience may stimulate endocannabinoid tone to release DA in mesolimbic areas, which feedbacks negatively on endocannabinoid levels (Di Marzo et al., 2009). However, after prolonged high-fat diet consumption and the development of obesity, this negative feedback may be impaired leading to chronically elevated endocannabinoid tone in accumbens shell. Consequently, DA stimulation may lead to both increased motivation to consume palatable foods and heightened reward after consumption.

Taken together, reward mechanisms implicated in the regulation of non-homeostatic food intake serve as potential central targets for the treatment of obesity. 


\section{Interactions between homeostatic and non-homeostatic hedonic mechanisms}

Anatomical and functional interactions exist between homeostatic and nonhomeostatic reward circuits that regulate food intake. Medial hypothalamic nuclei, part of the homeostatic circuit involved in the regulation of energy balance, are connected extensively with brain regions mediating reward and motivation (Berthoud, 2002). The arcuate nucleus of the hypothalamus directly projects to lateral hypothalamus, which is reciprocally connected to the limbic reward circuit (De Olmos and Heimer, 1999; Everitt and Robins, 2005). The central nucleus of the amygdala projects to lateral hypothalamus, and amygdala and lateral hypothalamus receive direct taste inputs from NTS (Berthoud, 2002). Other connections include projections from nucleus accumbens to VTA and lateral hypothalamus (Berthoud, 2002; Kelley and Berridge, 2002). Reciprocal connections between hypothalamic, brain stem and limbic reward circuits establish an anatomical framework for interactions and crosstalk between homeostatic and reward mechanisms in obesity.

\subsection{Adiposity signals: Leptin and insulin}

Peripherally-derived hormones, leptin and insulin, modulate feeding behavior, independent of actions in hypothalamus (Palmiter, 2007). In addition to widespread expression in hypothalamus, leptin and insulin receptors are co-localized on catecholaminergic neurons containing tyrosine hydroxylase, the rate limiting enzyme in DA and NE synthesis (Figlewicz et al., 2003), providing direct connections between peripheral adiposity signals and mesolimbic brain circuits that regulate motivational and hedonic elements of ingestive behavior.

Behavioral studies provide evidence of a role for leptin and insulin in food reward. Compared to control rats, CPP associated with a high-fat diet was blocked by leptin $(0.2 \mu \mathrm{g}$, ICV) or insulin (5 mU, ICV) (Figlewicz et al., 2004). Also, ICV infusion of leptin or insulin decreased PR breakpoints for sucrose self-administration in rats, compared to vehicle control (Figlewicz et al., 2006). Leptin and insulin reductions in motivation for food were absent in rats on a high-fat diet for $5 \mathrm{wk}$; such that, despite ICV leptin or insulin, rats fed a high-fat diet exhibited increased PR breakpoints for sucrose self-administration, compared to chowfed controls (Figlewicz et al., 2006). Thus, these adiposity signaling molecules modulate activity of neural circuits mediating reward and food-motivated behavior. Further, deficits in leptin and insulin signaling in reward circuits may underlie non-homeostatic hedonic food intake in obesity.

Behavioral paradigms also implicate DA as playing a major role in reward and motivation (Agmo et al., 1995; Figlewicz et al., 2001). Leptin receptor signaling in VTA modulates DA activity and food intake (Hommel et al., 2006). Leptin (3 mg/kg, IP) increases pSTAT3 expression, a marker of leptin signaling in VTA. pSTAT3 expression in VTA was robust following leptin infusion $(1 \mu \mathrm{g})$ directly into VTA, indicating that this was not likely the result of a primary effect in hypothalamus. Moreover, pSTAT3 was expressed in tyrosine hydroxylase-positive neurons, indicating that primarily DA neurons in VTA are responding 
to leptin. In anesthetized rats, leptin ( $2 \mathrm{mg} / \mathrm{kg}$, IV) reduces VTA DA neuronal firing, compared to control.

Evidence that VTA is important in leptin regulation of food reward and palatable food intake derives from viral-mediated RNA interference studies, generating rats with conditional gene knockdown of leptin receptors specifically in VTA (Hommel et al., 2006). Using two-bottle choice, leptin receptor knockdown rats consumed $50 \%$ more of a $0.2 \%$ sucrose solution compared to water, thus showing increased sensitivity to sucrose reward. In contrast, sucrose preference was absent in control rats. Also, leptin receptor knockdown increased palatable high-fat food intake, while high-fat food intake was not altered in controls. Leptin receptor knockdown selectively in midbrain augmented PR responding for sucrose, also supporting a role for midbrain leptin signaling in food-motivated behavior (Davis et al., 2011). Compared to control, leptin (15 ng/side, bilateral) injected into VTA elevated brain reward thresholds during intracranial self-stimulation (Bruijnzeel et al., 2011). Thus, leptin independently signals within reward circuits to decrease food reward and consumption of palatable foods.

Leptin regulation of DA reward pathway is evident in leptin deficient ob/ob mice (Fulton et al., 2006). In contrast to leptin's ability to suppress mesolimbic DA signaling (Hommel et al., 2006), leptin-deficient ob/ob mice show decreases in mesolmbic DA signaling. Compared to wild-type mice, ob/ob mice exhibit decreased tyrosine hydroxylase and phosphorylated tyrosine hydroxylase-ser40 expression, an indicator of tyrosine hydroxylase activity, in VTA and accumbens. Consistent with these findings, ob/ob mice exhibit decreased electrically-evoked accumbal DA release, compared to wild-type mice. In the presence of, nomifensine ( $3 \mu \mathrm{M}$; DAT inhibitor), a reduction in electrically-evoked DA signal was maintained, suggesting that DAT is not responsible for DA release deficits in ob/ob mice. In ob/ob mice, leptin (500 ng/h; $12 \mu \mathrm{g} /$ day, SC) corrected the decrease in tyrosine hydroxylase expression in VTA and accumbens and phosphorylated tyrosine hydroxylase-ser40 in accumbens. However, leptin's ability to correct defects in accumbens DA release in ob/ob mice was not determined. Discrepancies found in ob/ob mice may be attributed to compensatory changes resulting from a long-term leptin deficiency (Opland et al., 2010). Thus, mesolimbic system hypoactivity may underlie compensatory overeating in leptin deficient ob/ob, enhancing a deficient DA system. Overall, these results suggest that reward deficits found in obesity (Geiger et al., 2008), may be attributed to deficient leptin signaling in reward circuits.

Given synaptic connections between lateral hypothalamus and the mesolimbic DA system including VTA and nucleus accumbens, the role of lateral hypothalamus leptin receptors in regulating mesolimbic DA function also was evaluated in ob/ob mice (Leinninger et al., 2009). Leptin (250 pg) injection into lateral hypothalamus decreased feeding, increased VTA tyrosine hydroxylase expression and increased accumbens DA content in ob/ob mice, compared to vehicle control ob/ob mice. These results suggest that leptin acts via lateral hypothalamus neurons expressing leptin receptors to modulate mesolimbic DA function.

Leptin resistance predisposes rodents to high-fat DIO. Rats with pre-existing leptin resistance due to leptin overexpression or age-related leptin resistance, have exacerbated excessive food consumption and weight gain upon subsequent exposure to a high-fat diet, 
compared with leptin responsive rats (Scarpace et al., 2005; Judge et al., 2008; Scarpace and Zhang, 2009). Given the role of VTA leptin receptors in decreasing food reward and motivation for palatable food, disruption in leptin function in VTA suggests an increased vulnerability to palatable foods and high-fat diet-induced weight gain (Hommel et al., 2006). Leptin resistance in VTA of DIO rats was determined by pSTAT3 expression (Matheny et al., 2011). Relative to chow-fed controls, increased leptin resistance in VTA and arcuate of DIO rats (evidenced by decreased pSTAT3 expression), provides a potential mechanism for increased susceptibility of DIO rats to palatable food consumption and the development of obesity (Matheny et al., 2011).

A role for DA in modulating leptin in humans comes from a single-blind crossover study conducted in obese women assigned to either bromocriptine ( $5 \mathrm{mg}$; oral) or placebo treatment for 8 days (Kok et al., 2006). Compared to placebo, bromocriptine decreased circulating leptin levels, suggesting that DA is involved in control of leptin release in humans. The ability of bromocriptine to modulate palatable food consumption and restore leptin function in hypothalamic and reward circuits will increase our understanding of reciprocal interactions between leptin and D2 receptor signaling.

DAT is a potential cellular target for insulin modulation of reward circuits (Jaber et al., 1997). Central administration of insulin in rats increases DAT mRNA expression in VTA/ substantia nigra compacta, compared with DAT in vehicle controls (Figlewicz et al., 1994, 2003). A functional consequence of increased DAT expression is decreased DA signaling that further decreases palatable food reward. To elucidate contributions of insulin signaling on VTA DA, insulin's effect on electrically-evoked DA release in VTA was determined using fast-scan cyclic voltammetry in mice (Mebel et al., 2012). In a concentrationdependent manner, insulin (10-1000 nM; bath-applied) attenuated electrically-evoked DA release from VTA slices. Insulin receptor activation triggers a variety of signal transduction cascades involving activation of phosphatidylinositol-3 kinase pathway, that triggers activation of the mammalian target of rapamycin (Taha and Klip, 1999). Activation of phosphatidylinositol-3 kinase increases DAT trafficking (Simon et al., 1997; Carvelli et al., 2002; Garcia et al., 2005). Insulin (500 nM) failed to attenuate electrically-evoked DA release when VTA slices were pre-incubated with either a phosphatidylinositol-3 kinase inhibitor, wortmannin $(100 \mathrm{nM})$, or an inhibitor of the mammalian target of rapamycin (50 nM) (Mebel et al., 2012), suggesting that phosphatidylinositol-3 kinase and rapamycin signaling are necessary for insulin suppression of DA release in VTA. Insulin (500 nM) suppression of DA release was abolished by GBR 12909 (1-[2-[bis(4fluorophenyl)methoxy]ethyl]-4-(3-phenylpropyl)-piperazine; $500 \mathrm{nM}$; DAT inhibitor) and in mice lacking DAT (Mebel et al., 2012). However, insulin's effect in mice lacking the NE transporter was not different from wild-type mice, suggesting that DAT plays a role in the underlying mechanism responsible for the insulin-mediated decrease in DA release in VTA.

Insulin effects in VTA, specifically on palatable food consumption, was evaluated in sated mice (Mebel et al., 2012). Insulin (0.3 $\mu \mathrm{g} / 0.2 \mu \mathrm{l})$ or vehicle was administered intra-VTA after mice consumed regular chow during 4-h access, and prior to a 1-h exposure to palatable sweetened high-fat food. Control mice consumed a similar quantity of sweetened high-fat food as during the first hour of regular chow access, indicating that mice consume 
palatable food even when sated. In contrast, insulin reduced the amount of sweetened highfat food consumed during the 1-h exposure. Thus, insulin acting in VTA inhibits sated consumption of palatable food (Mebel et al., 2012). The insulin-mediated decrease in DA release in VTA may be the mechanism underlying the reduction in palatable food salience once satiety is attained. Insulin signaling in VTA producing a decrease reward is supported by an elevated brain reward threshold (intracranial self-stimulation) following intra-VTA insulin (0.005 mU/side) (Bruijnzeel et al., 2011). Thus, disrupted insulin function in VTA may promote hedonic intake of palatable foods, and serve as a mechanism underlying reward and contributing to obesity. Overall, leptin and insulin not only modulate satiety and metabolism, but also decrease food reward. This ability to influence homeostatic and nonhomeostatic hedonic mechanisms underlying obesity makes central leptin and insulin receptors attractive targets for intervention.

\subsection{Hunger signal: Ghrelin}

Ghrelin produces a potent orexigenic effect when food is available (Wren et al., 2000). In addition to homeostatic regulation of energy balance, ghrelin modulates DA reward circuitry and influences motivational and reinforcing aspects of palatable foods, promoting hedonic food consumption (Jerlhag et al., 2007; Skibicka et al., 2011, 2012a). Ghrelin influences the responsiveness of brain regions involved in processing food cues in humans. Using fMRI, cerebral response to food and non-food (scenery) images was determined following singleblinded ghrelin infusion ( $1 \mu \mathrm{g} / \mathrm{kg}$ ) in healthy humans (Malik et al., 2008). BOLD response to visual food cues was increased in amygdala, orbitofrontal cortex, anterior insula and striatum. Also, self-reports of hunger were increased in ghrelin vs. control conditions, and were correlated positively with ghrelin-induced increases in amygdala, orbitofrontal cortex and pulvinar activity. These regions encode salience, hedonic and incentive value of visual cues. Thus, ghrelin enhances palatable food consumption by increasing the hedonic response to food-related cues.

Similar to leptin and insulin, ghrelin modulates VTA DA activity and alters behaviors associated with mesolimbic reward circuitry. Ghrelin (30 $\mu$; IP) produces synaptic remodeling in VTA DA cells in mice in a GHSR-dependent manner (Abizaid et al., 2006). Compared to saline control mice, ghrelin increased frequency of miniature excitatory postsynaptic currents and decreased the frequency of miniature inhibitory postsynaptic currents in VTA DA neurons. These ghrelin effects were absent in GHSR-deficient mice. Using another approach, infusion of ghrelin $(0.5 \mu \mathrm{g} / 0.5 \mu \mathrm{l})$ directly into VTA in awake rats increased food intake for $2 \mathrm{~h}$ (Abizaid et al., 2006). Ghrelin (5 $\mu \mathrm{g}$, IP) induced feeding responses in rats that were attenuated by intra-VTA infusions of GHSR antagonist, BIM28163 (4-[[(2S)-2-[[(2R)-2-[[(2R)-3-(1-benzothiophene-3-yl)-2-(piperidine-3carbonylamino)propanoyl]amino]-3-(1H-indol-3-yl)propanoyl]amino]-3phenylpropanoyl]amino]piperidine-4-carboxamide; $0.5 \mu \mathrm{g} / 0.5 \mu \mathrm{l}$ ) given $1 \mathrm{~h}$ prior to ghrelin (Abizaid et al., 2006). Thus, circulating ghrelin reaches VTA in physiologically relevant amounts to elicit a feeding response via activation of mesolimbic DA circuitry.

Ghrelin $(1 \mu \mathrm{g} / 1 \mu \mathrm{l})$ infusion into VTA increases DA release from accumbens core in freely moving mice (Jerlhag et al., 2007). Infusion of ghrelin (0.33 and $1 \mu \mathrm{g} / 0.5 \mu \mathrm{l})$ into VTA 
increases motivation for sucrose reward assessed using the PR schedule of reinforcement (Skibicka et al., 2011, 2013). However, increases in motivation for sucrose reward were absent in rats receiving ghrelin in accumbens core, suggesting that VTA is the target for ghrelin's action on food motivation. Pretreatment with D1 receptor antagonist, SCH-23390 or D2 receptor antagonist, eticlopride ( $1 \mu \mathrm{g} / 0.5 \mu \mathrm{l}$; intra-accumbens shell) attenuated ghrelin-induced increases in motivation to seek sucrose, but not chow consumption. Therefore, VTA-accumbens DA signaling mediates ghrelin's effect on effort-based food reward seeking, but not food intake.

A cholinergic-DA link is implicated in ghrelin-induced increase in motivation for food reward (Dickson et al., 2010). Ghrelin ( $1 \mu \mathrm{g} / \mu \mathrm{l}$, ICV)-induced food intake in mice was suppressed by mecamylamine ( $2 \mathrm{mg} / \mathrm{kg}$, IP), a non-selective nicotinic receptor antagonist, but not by hexamethonium $(2 \mathrm{mg} / \mathrm{kg}$, IP), a peripheral nicotinic receptor antagonist (Dickson et al., 2010), indicating central effects of mecamylamine on ghrelin-induced food intake. Mecamylamine ( $2 \mathrm{mg} / \mathrm{kg}$, IP) blocked food intake induced by ghrelin infusion $(1 \mu \mathrm{g} / 0.5 \mu \mathrm{l})$ into VTA and blocked palatable food-induced CPP in rats. However, ability of ghrelin to rescue CPP for palatable food was not assessed. These results suggest that ghrelin-induced food intake is mediated via nicotinic receptors and nicotinic receptor antagonists decrease rewarding properties of food.

NPY and opioid modulation of ghrelin's effects on food motivated behavior has been investigated (Skibicka et al., 2012b). Inhibitory effects of a selective NPY Y1 receptor antagonist, LY 1229U91 (1-12 $\mu \mathrm{g} / 2 \mu \mathrm{l})$, or opioid receptor antagonist, naltrexone (50 $\mu \mathrm{g} / 2$ $\mu \mathrm{l})$, injected either ICV or intra-VTA on ghrelin $(1 \mu \mathrm{g} / 2 \mu \mathrm{l}, \mathrm{ICV})$-induced increases in food motivated behavior were assessed using a PR reinforcement schedule. Ghrelin-induced increases in sucrose-motivated behavior were blocked by ICV pre-treatment with LY 1229U91 and naltrexone. However, ghrelin effects were blocked only by intra-VTA naltrexone, not by LY 1229U91 (Skibicka et al., 2012b). These findings suggest that opioid signaling in VTA mediates ghrelin effects on food reward. Thus, ghrelin, an important gutbrain signal, promotes food intake not only in response to energy deficit, but also to reward from palatable foods.

\subsection{Satiety signal: GLP-1}

GLP-1, an incretin hormone and peripherally-derived satiety signal, has been linked centrally to non-homeostatic and motivational processes associated with food reward (Alhadeff et al., 2012; Dickson et al., 2012; Skibicka, 2013). In addition to homeostatic centers, GLP-1 receptors are expressed in VTA and accumbens (Merchenthaler et al., 1999; Alhadeff et al., 2012). Peripheral administration of exendin-4, a synthetic GLP-1 analog, activates Fos in accumbens (Gu et al., 2013), suggesting that GLP-1 modulates hedonic feeding behavior. Compared to vehicle control, exendin-4 (0.03-3 $\mu \mathrm{g} / 1 \mu \mathrm{l}, \mathrm{ICV})$ dosedependently decreased PR responding for sucrose reinforcement (Dickson et al., 2012). Pretreatment with exendin-3 (20 $\mu \mathrm{g} / \mu \mathrm{l}$, ICV; a selective GLP-1 receptor antagonist) reversed the exendin-4-induced $(0.2 \mu \mathrm{g} / \mu \mathrm{l}, \mathrm{ICV})$ reduction in motivation to obtain sucrose reinforcement, further confirming a role of central GLP-1 receptors in food-motivated behavior. Both intra-VTA $(0.03$ and $0.1 \mu \mathrm{g} / 0.5 \mu \mathrm{l})$ and intra- accumbens $(0.1 \mu \mathrm{g} / 0.5 \mu \mathrm{l})$ 
infusion of exendin-4 decreased motivation for sucrose reinforcement, compared to vehicle control (Dickson et al., 2012). Thus, these findings are consistent with mesoaccumbens GLP-1 signaling playing a role in food reward.

GLP-1 receptor activation in NTS-induced suppression of food reward suggests that neurobiological targets underlying GLP-1-mediated food reward are not limited to the DA mesolimbic system. Exendin-4 $(0.05 \mu \mathrm{g} / 0.3 \mu \mathrm{l})$ infusion into rat NTS resulted in selective reduction in palatable food intake, relative to chow (Richard et al., 2015). Compared to vehicle-control, exendin-4 (0.05 $\mu \mathrm{g} / 0.3 \mu \mathrm{l})$ infusion into NTS resulted in less time in the sucrose-paired chamber in the CPP test, suggesting that NTS GLP-1 signaling decreases palatable food reward. GLP-1 $(2.0 \mu \mathrm{g} / 0.3 \mu \mathrm{l})$ and exendin-4 $(0.05$ or $0.1 \mu \mathrm{g} / 0.3 \mu \mathrm{l})$ injected into NTS suppressed motivation to obtain sucrose reward assessed using a PR schedule of reinforcement. GLP-1 receptor stimulation with exendin-4 (0.05 $\mu \mathrm{g} / 0.3 \mu \mathrm{l})$ infusion into NTS resulted in a 4-fold increase in tyrosine hydroxylase mRNA expression and 2-fold increase in D2 receptor mRNA expression in VTA. These findings suggest that NTS GLP-1 receptor activation decreases food reward and motivation for palatable food by modulating mesolimbic DA activity (Richard et al., 2015). Further understanding of interactions between NTS GLP-1 and mesolimbic DA in the regulation of non-homeostatic hedonic food intake is needed.

GLP-1 receptor agonist effects were evaluated recently in a randomized crossover study in obese humans with type- 2 diabetes treated for 10 days with either the GLP-1 receptor agonist, liraglutide (1.8 mg, SC), or insulin glargine (100 units/ml, IM), and brain response to pictures of food were assessed using fMRI (Ten Kulve et al., 2015). Under a fasted state, liraglutide decreased BOLD responses in insula and putamen upon viewing food pictures, compared with those treated with insulin glargine. Insula is involved in processing and evaluation of food cues and in craving for food (Small et al., 2001; Pelchat et al., 2004).

These results suggest that liraglutide-induced decreases in food reward and perhaps craving contribute to reduction of palatable food consumption. Thus, central GLP-1 receptors are potential therapeutic targets for obesity that modulates reward mechanisms underlying palatable food consumption.

\subsection{Orexins and $\mathrm{MCH}$}

In addition to homeostatic processes, the lateral hypothalamic orexin system is involved in non-homeostatic reward-driven feeding behavior (Borgland et al., 2009; Cason et al., 2010; Cason and Aston-Jones, 2013; Williams, 2014). Orexin neurons project locally within hypothalamus and widely throughout brain, including projections to VTA and locus coeruleus (Peyron et al., 1998). Both orexin receptor subtypes, orexin-1 and orexin-2, are located in reward relevant brain regions including DA projections from VTA to accumbens, prefrontal cortex and amygdala (Sakurai et al., 1998; Marcus et al., 2001). Orexin-1 receptors are involved predominantly in reward and feeding behaviors (deLecea et al., 1998; Sakurai et al., 1998). Orexin-1 receptor antagonist, SB334867 (10 mg/kg, IP) decreased the PR breakpoint for high-fat chocolate flavored pellets, relative to vehicle control mice (Borgland et al., 2009), revealing a role for this receptor subtype in motivation for palatable food. 
The role of orexin signaling in compulsive binge consumption of palatable food was assessed using a binge model in rats (Picolli et al., 2012). Binge eating of highly palatable food was evoked by 3 cycles of food restriction, stress elicited by exposure to palatable food for $15 \mathrm{~min}$ (rats see the food, but do not have access), and finally access to palatable food. Effects of a selective orexin-1 receptor antagonist, GSK1059865 ([(2S,5S)-2-[[(5bromopyridin-2-yl)amino]methyl]-5-methylpiperidin-1-yl]-(3-fluoro-2-methoxyphenyl)methanone; 10 and $30 \mathrm{mg} / \mathrm{kg}, \mathrm{PO}$ ), a selective orexin-2 receptor antagonist, JNJ-10397049 (1-(2,4-dibromophenyl)-3-[(4S,5S)-2,2-dimethyl-4-phenyl-1,3-dioxan-5-yl]urea; 1 and 3 $\mathrm{mg} / \mathrm{kg}$, IP), and a dual orexin-1/orexin-2 receptor antagonist, SB649868 ( $N-[((2 S)-1-\{[5-(4-$ fluorophenyl)-2-methyl-1,3-thiazol-4-yl]carbonyl\}-2-piperidinyl)methyl]-1-benzofuran-4carboxamide; 1 and $3 \mathrm{mg} / \mathrm{kg}$, PO) were determined. Compared to vehicle control, GSK1059865 and SB649868 decreased palatable food intake, however, JNJ-10397049 did not alter feeding behavior, suggesting that orexin-1 receptors, but not orexin-2 receptors, mediate binge eating. Given that binge eating contributes to obesity, orexin-1 receptors are a target for decreasing binge eating of palatable foods.

Immunohistochemistry studies show orexin-1 receptor expression and orexin neuron projections to the dorsal vagal complex, including NTS, area postrema and dorsal motor nucleus of the vagus (Peyron et al., 1998; Hervieu et al., 2001; Marcus et al., 2001; Zheng et al., 2005). The role of caudal brainstem orexin- 1 receptors in food reward was evaluated in rats by determining effects of fourth cerebral ventricle infusion of orexin-A and a orexin-1 receptor antagonist, SB334867, on PR responding for sucrose pellets and on expression of high-fat diet induced CPP (Kay et al., 2014). Orexin-A $(1 \mathrm{nmol} / 2 \mu \mathrm{l})$ robustly increased the breakpoint for sucrose reinforcement, compared to vehicle. SB334867 (20 nmol/2 $\mu \mathrm{l})$ decreased the breakpoint to obtain sucrose reinforcement and inhibited CPP. These results suggest that hindbrain orexin-1 receptor activity modulates food-motivated behavior and plays a role in the response to environmental cues assocated with palatable food.

Excessive consumption of palatable foods contributes to obesity. While people attempt to control food intake through healthy choices including dieting, most relapse to unhealthy eating occurs after acute exposure to palatable foods, associated cues or stress (Peterson and Mitchell, 1999; Torres and Nowson, 2007). The reinstatement paradigm, which commonly is used to study relapse to seeking abused drugs, has been employed to study relapse to seeking palatable food (Self and Nestler, 1998; Shaham et al., 2003; Ghitza et al., 2006). In the reinstatement paradigm, food-restricted rats are trained using operant procedures to obtain palatable food reinforcement, typically employing a fixed ratio schedule (Calu et al., 2014). Following food pellet training and extinction of the food-reinforced responding, palatable food seeking behavior is reinstated by priming with palatable food pellets, food-associated cues, or stress, e.g., induced by yohimbine administration (Bremner et al., 1996). A role for orexin-1 receptor signaling in reinstatement of palatable food seeking behavior is supported by results showing that the orexin-1 receptor antagonist, SB334867 (5 and $10 \mathrm{mg} / \mathrm{kg}$, IP), decreased yohimbine-induced reinstatement of sucrose seeking in food-sated rats trained to self-administer 5\% sucrose solution (Richards et al., 2008). Pretreatment with SB334867 (30 $\mathrm{mg} / \mathrm{kg}$, IP) decreased cue (tone and light)-induced reinstatement of saccharin-seeking behavior, which had been extinguished, in both sated and food-restricted rats (Cason and Aston-Jones, 2013). These results suggest that orexin-1 signaling plays a role in motivation 
to obtain palatable food and in the response to cues previously associated with palatable foods.

Orexin-1 signaling mediates cue-induced feeding in rats (Cole et al., 2015). Through repeated pairings of a neutral stimulus (a tone) with delivery of palatable food, rats learn that the cue predicts food availability. Ability of cue to stimulate overeating is assessed later in sated rats by presenting the tone and measuring food consumption. In the presence of cue, rats eat greater amounts of food. SB334867 (20 mg/kg, IP), an orexin-1 receptor antagonist, had no effect on baseline eating, but reduced cue-induced consumption in sated rats, while activating Fos in medial prefrontal cortex and paraventricular thalamus. Thus, orexin signaling through cortical and thalamic sites drives cue-induced eating in the absence of hunger. Orexin-1 receptor antagonists appear beneficial in decreasing motivation for palatable food reward and preventing relapse to palatable food seeking behavior and serve as targets for therapeutics.

Subsets of orexin neurons in lateral hypothalamus secrete the orexigenic neuropeptide $\mathrm{MCH}$ and have input to accumbens (Georgescu et al., 2005; Sears et al., 2010). The role of MCH-1 receptors in cue-potentiated feeding of palatable food is supported by findings that sated MCH-1 receptor knockout mice fail to overconsume sucrose in response to a conditioned stimulus, relative to sated wild-type mice (Sherwood et al., 2015). Thus, cue-potentiated feeding requires intact $\mathrm{MCH}-1$ receptor signaling. $\mathrm{MCH}-1$ receptor antagonists may be beneficial to reduce excessive drive to consume high salience food reinforcers. MCH-1 receptors also appear to be excellent pharmacologic targets for therapeutic discovery.

In summary, homeostatic circuits interact functionally with the reward circuitry in brain, underscoring that both systems are important in regulation of food intake. Understanding the functional interaction between homeostatic and reward systems will provide anti-obesity strategies that address food intake from energy balance and hedonic perspectives. Discoveries highlighting the impact of homeostatic signals (leptin, insulin and GLP-1) on the reward system extend the potential therapeutic range of these targets. For example, GLP-1 receptors mediate aspects of palatable food consumption including satiety, reward and motivation. Thus, preclinical and clinical evaluation of these targets should investigate therapeutic candidates for effects on energy balance (decreases in food intake and weight loss) and non-homeostatic hedonic mechanisms (motivation for palatable foods and relapse to food-seeking behavior). Such targets may offer long-term efficacy in the treatment of obesity.

\section{Adiposity and hunger signals: Vulnerability to drug abuse}

Adiposity signals modulate mesolimbic DA reward circuits and regulate nonhomeostatic hedonic food intake. Given overlapping neuronal circuits in obesity and substance use disorders (Volkow et al., 2008), these peripherally-derived signals also alter sensitivity to reward produced by drug reinforcers, including nicotine and alcohol. For example, smoking rates in adults with diabetes are greater than in the general population (Bishop et al., 2009). Furthermore, tobacco use is a risk factor that promotes negative health consequences of type-2 diabetes (Cho et al., 2009). Given epidemic increase in type-2 diabetes, a metabolic 
sequela of obesity, diabetes-induced changes in reward pathways may increase susceptibility to tobacco use.

As a model of diabetes, streptozotocin treatment produces toxic effects on pancreatic- $\beta$ cells and induces hypoinsulinemia and hyperglycemia. In streptozotocin-treated rats, IV selfadministration using a fixed ratio- 1 schedule of reinforcement and extended access ( $23 \mathrm{~h})$ to increasing nicotine doses $(0.03-0.09 \mathrm{mg} / \mathrm{kg} / 0.1 \mathrm{ml}$ infusion) revealed increased nicotine intake, compared to vehicle control rats (O'Dell and Koob, 2007; O'Dell et al., 2014). Highfat diet models of obesity resemble the etiology of type-2 diabetes, as animals develop insulin resistance and hyperglycemia (Woods et al., 2003). Using another diabetes model, the magnitude of nicotine CPP was greater in insulin-resistant rats fed a high-fat diet for 5 wk compared to regular diet (Richardson et al., 2014). Further, nicotine CPP was absent in rats fed regular diet and in non-insulin-resistant rats fed high-fat diet. Nicotine reward was exacerbated in rats that received the high-fat diet and displayed insulin resistance, suggesting an enhancement of nicotine reward via disruption of insulin signaling. Thus, individuals with diabetes may experience greater nicotine reward, augmenting the risk for tobacco addiction.

Peripherally-derived adiposity and hunger signals alter the sensitivity to nicotine and alcohol reward. Human studies reveal a positive correlation between circulating leptin levels and tobacco craving in smokers after $24 \mathrm{~h}$ of abstinence (Von der Goltz et al., 2010; al'Absi et al., 2011), suggesting that leptin modulates DA reward circuits during nicotine withdrawal to facilitate nicotine seeking. Further investigation of links between appetite regulation, obesity and nicotine dependence is warranted.

Similarly, central ghrelin signaling is implicated in alcohol reward (Jerlhag et al., 2009). Ghrelin ( $2 \mu \mathrm{g}$, ICV, intra-VTA or laterodorsal tegmental area) increased alcohol (10\% alcohol in water) consumption in a 2-bottle choice limited access paradigm in mice (Jerlhag et al., 2009). Alcohol intake was suppressed by GHSR1A antagonists, BIM28163 (5 $\mu \mathrm{g}$, ICV) or JMV2959 (2-amino-N-[(1R)-2-(1H-indol-3-yl)-1-[4-[(4-methoxyphenyl)methyl]-5(2-phenylethyl)-1,2,4-triazol-3-yl]ethyl]acetamide, $6 \mathrm{mg} / \mathrm{kg}$; IP). Alcohol produced CPP in wild-type mice and GHSR1A antagonists blocked alcohol-induced CPP. Alcohol-induced CPP was not found in GHSR1A knockout mice. Thus, central ghrelin signaling via GHSR1A mediates alcohol reward.

Recent human studies emphasize ghrelin's role in alcohol abuse. In a 12-wk study conducted in alcohol-dependent humans, plasma ghrelin was correlated positively to alcohol intake and craving (Leggio et al., 2013). These results support a role for ghrelin in alcohol-seeking behavior. Effects of ghrelin (1, $3 \mu \mathrm{g} / \mathrm{kg}$, IV) or placebo on alcohol craving (assessed using a visual analog scale) in alcohol-dependent, non-treatment-seeking, heavy-drinkings humans were determined in a double-blind, placebo-controlled cue-reactivity study (Leggio et al., 2014). A positive correlation between cue-induced alcohol craving and blood ghrelin was found, suggesting that ghrelin plays a role in facilitating alcohol-seeking behavior. Further, ghrelin ( $3 \mu \mathrm{g} / \mathrm{kg}$, IV) decreased serum leptin levels in non-treatment-seeking, alcoholdependent, heavy drinkers, compared with vehicle control (Haass-Koffler et al., 2015). Moreover, ghrelin-induced decreases in leptin levels correlated negatively with increased 
alcohol craving in heavy drinkers, suggesting that leptin and ghrelin exert opposite effects on alcohol seeking behavior. Thus, ghrelin and leptin interact to influence alcohol craving and are potential therapeutic targets for alcohol addiction.

In summary, the role of adiposity and hunger signals extends beyond homeostatic and nonhomeostatic regulation of food intake, such that these signals are involved in mediating reward sensitivity to drug reinforcers. Further studies are needed to establish the links between obesity and drug abuse vulnerability.

\section{Pharmacotherapies for obesity}

A healthy diet and exercise are beneficial for both prevention and treatment of obesity. However, lifestyle modification alone has failed to ameliorate the obesity epidemic, necessitating the development and implementation of effective pharmacotherapeutic strategies. Based on FDA guidelines, an anti-obesity medication is deemed effective if the drug produces $>5 \%$ reduction in body weight compared to placebo, or if at least $35 \%$ of study participants lose $>5 \%$ of their baseline body weight (FDA draft guidance, 2007). A $5-10 \%$ weight loss reduces the incidence of both type 2 diabetes and cardiovascular complications (Avenell et al., 2004). Current pharmacotherapeutics and candidates under clinical development for obesity are categorized herein as follows: 1) medications that act peripherally to impair dietary fat absorption; 2) medications that act centrally to decrease food intake; and 3) medications that facilitate energy expenditure. Despite compliance with weight loss guidelines, the majority of drugs launched for the treatment of obesity over the last two decades have been withdrawn due to safety issues associated with increased risk of cardiovascular and psychiatric complications. The failure of these medications is attributed to the multifactorial pathogenesis and the complex neuro-hormonal regulation of energy balance. Although some efficacy has been found with monotherapies that target a single protein or pathway involved in obesity, physiological counter-regulation mechanisms involving alternate pathways pose major limitations (Rodgers et al., 2012). Consequently, a disease with multiple etiologies, requires a multi-target approach. In this context, two most recently approved combination therapies for obesity include Qsymia ${ }^{\circledR}$, a combination of phentermine and topiramate, and Contrave ${ }^{\circledR}$, a combination of naltrexone and bupropion. Importantly, combination therapies are postulated to have increased efficacy through a synergistic action that counteracts compensatory mechanisms, decreases adverse effects and increases tolerability with low dose combinations (Gadde and Allison, 2009). This section discusses FDA approved anti-obesity medications (Table 1), therapeutic candidates undergoing clinical development (Table 2), and compounds that have potential as therapeutic candidates based on findings from preclinical and preliminary human studies (Table 3).

\subsection{Approved anti-obesity drugs}

6.1.1. Phentermine (Adipex-P®)-Phentermine (2-methyl-1-phenylpropan-2-amine) is a sympathomimetic amine approved by the FDA in 1959 for short-term ( $\leq 2 \mathrm{wk}$ ) weight management as an adjunct to caloric restriction and lifestyle modifications for obese and overweight individuals with weight-related comorbidities (Sweeting et al., 2014). Phentermine is the most commonly prescribed anti-obesity medication in the United States 
(Hampp et al., 2013). In a randomized, double-blind, placebo-controlled 12-wk trial, the safety and efficacy of phentermine $(30 \mathrm{mg}$ ) in a diffused-controlled release formulation was assessed in obese participants with controlled diabetes, hypertension or dyslipidemia (Kang et al., 2010). Compared to placebo, participants given phentermine exhibited significant body weight reductions ( 8 vs. $2 \mathrm{~kg}$ ). Total cholesterol and low-density lipoprotein cholesterol levels also were improved with phentermine. No between-group differences in systolic and diastolic blood pressure were found.

Frequently, phentermine is prescribed off-label for longer than the FDA approved period (Hampp et al., 2013). Physicians typically prescribe the generic $37.5 \mathrm{mg}$ tablet and advise patients to administer a half tablet (18.75 mg) mid-morning, once daily (Adipex-P prescribing information, 2012). Despite a long history of use, limited information is available with respect to controlled trials demonstrating efficacy of phentermine monotherapy for a year or more or regarding risk for cardiovascular disease. The longest (36 wk) placebo-controlled trial evaluating 108 obese women treated with phentermine $30 \mathrm{mg}$ per day administered either continuously or intermittently (alternating months) reported a mean weight loss of 12.2 and $13.0 \mathrm{~kg}$, respectively, compared with $4.8 \mathrm{~kg}$ given placebo (Munro et al., 1968). However, only 59\% of participants completed the trial, underscoring the need for controlled long-term studies to demonstrate safety and efficacy. The most common adverse events include dry mouth and insomnia (Yanovski and Yanovski, 2014). Serious complications include palpitations, tachycardia and hypertension (Sweeting et al., 2014). Long-term, dose-ranging clinical trials are needed to confirm the safety and efficacy of phentermine as an anti-obesity therapeutic.

6.1.2. Orlistat (Xenicaß囚)-Orlistat ((S)-((S)-1-((2S, 3S)-3-hexyl-4-oxooxetan-2yl)tridecan-2-yl) 2-formamido-4-methylpentanoate) is the first gastrointestinal lipase inhibitor approved by the FDA in 1999 for the treatment of obesity (McNeely and Benfield, 1998; Heal et al., 2012). Dietary fat is metabolized by gastric and pancreatic lipases into absorbable free fatty acids and monoglycerides, facilitating intestinal absorption. Orlistat inactivates gastrointestinal lipase, reducing the absorption of dietary fat (Hogan et al., 1987). Obese individuals treated with orlistat ( $360 \mathrm{mg} /$ day, in 3 divided doses) for $12 \mathrm{wk}$ and maintained on a hypocaloric diet had greater body weight loss compared with placebo (5\% vs. 3.5\%; McNeely and Benfield, 1998). In several randomized controlled trials, orlistat exhibited long-term (2-4 years) efficacy for body weight reduction (Hauptman et al., 2000; Heymsfield et al., 2000; Torgerson et al., 2004). Importantly, orlistat improved blood pressure, insulin resistance and serum lipid levels (Torgerson et al., 2004; Siebenhofer et al., 2009).

Commonly experienced gastrointestinal side-effects of orlistat include diarrhea, flatulence, bloating, abdominal pain and dyspepsia (Siebenhofer et al., 2009). Often, daily multivitamins are co-prescribed to prevent fat-soluble vitamin deficiencies (Padwal and Majumdar, 2007). Following oral administration, orlistat is excreted almost entirely in the feces within 3-5 days, undergoes minimal systemic absorption and exhibits no accumulation (Zhi et al., 1996; Sjostrom et al., 1998). Several cases report acute kidney injury in orlistat users predisposed to metabolic conditions such as diabetes (Courtney et al., 2007;

Karamadoukis et al., 2009). Upon renal biopsy, increased urinary oxalate and oxalate crystal 
deposition were found (Ferraz et al., 2004; Sarica et al., 2008). Oxalate crystal deposition has been attributed to unabsorbed dietary fat binding to calcium in the gut lumen, which competitively inhibits calcium binding of oxalate, which is absorbed and deposited in renal parenchyma (Karamadoukis et al., 2009). In 2012, a safety label warning for orlistat was approved, which indicates that some patients may develop increased urinary oxalate levels and that renal function should be monitored in those at risk for renal insufficiency. From 1999 to 2008, the FDA received 32 reports of serious liver injury in patients using orlistat, including 6 cases of liver failure (FDA drug safety communication, 2010). In May 2010, a warning of severe liver injury was included. Nevertheless, orlistat provides an overall reasonable benefit to risk ratio for the treatment of obesity. Given the established safety profile of orlistat, a 60-mg over-the-counter dose was approved as a weight loss aid for overweight adults along with a reduced-calorie, low-fat diet and exercise program (FDA, 2007). The 60-mg dose is tolerated better than the 120-mg dose as gastrointestinal sideeffects are minimal when individuals consume less than $30 \%$ of their energy from fat (Anderson, 2007).

6.1.3. Lorcaserin (Belviq®) -5-HT has a role in the short-term regulation of food intake and its actions are implicated in satiety (Blundell, 1977). Dexfenfluramine, an inhibitor of the 5-HT transporter, was approved initially as an appetite suppressant for obesity, but subsequently withdrawn due to serious adverse side-effects including hallucinations induced by $5-\mathrm{HT}_{2 \mathrm{~A}}$ receptor activation, heart valve disease (cardiac valvulopathy), and pulmonary artery hypertension induced by $5-\mathrm{HT}_{2 \mathrm{~B}}$ receptor activation (Weissman et al., 1998; Launay et al., 2002). In 2012, the FDA approved lorcaserin, ((1R)-8-chloro-1-methyl-2, 3, 4, 5tetrahydro-1H-3-benzazepine), a selective $5-\mathrm{HT}_{2 \mathrm{C}}$ agonist, and a milestone therapeutic for the treatment of obesity. About 13 years after the approval of orlistat, lorcaserin was launched as the second anti-obesity drug. Importantly, lorcaserin exhibits high selectivity for 5- $\mathrm{HT}_{2 \mathrm{C}}$ receptors and is devoid of adverse effects associated with 5- $\mathrm{HT}_{2 \mathrm{~A}}$ and 5- $\mathrm{HT}_{2 \mathrm{~B}}$ receptor activation (Martin et al., 2011). Anti-obesity effects of lorcaserin are regulated by 5$\mathrm{HT}_{2 \mathrm{C}}$ receptors that are expressed on POMC neurons of arcuate nucleus and their activation results in increased satiety (Lam et al., 2008). Mice lacking melanocortin MC4R are not responsive to 5- $\mathrm{HT}_{2 \mathrm{C}}$ agonist-induced hypophagia, suggesting that $5-\mathrm{HT}_{2 \mathrm{C}}$ receptor agonists, such as lorcaserin, promote hypophagia by downstream activation of the melanocortin pathway.

The FDA approved lorcaserin at a dose of $10 \mathrm{mg}$ twice daily when supplemented by a reduced calorie diet and increased physical activity for patients with a body mass index of $\geq 30$, or $27 \mathrm{~kg} / \mathrm{m}^{2}$, with at least one weight-related comorbidity, such as hypertension, type 2 diabetes or dyslipidemia (FDA, 2012a). The approved labeling indicates that at wk 12, if body weight is not decreased by $5 \%$ or more, lorcaserin treatment should be discontinued (Taylor et al., 2013). Safety and efficacy of lorcaserin for weight loss in obese and overweight patients with type 2 diabetes in conjunction with a lifestyle modification program were evaluated in a 1-year, randomized, double-blind, placebo-controlled trial (O'Neil et al., 2012). In this study, 38\% of participants treated with lorcaserin (10 mg, twice daily) exhibited at least a 5\% reduction in body weight (O'Neil et al., 2012). The weight reduction was evident at $2 \mathrm{wk}$, and remained greater in the lorcaserin group than in the 
placebo group throughout the study. At wk 52, participants treated with lorcaserin exhibited greater body weight loss compared to those treated with placebo (4.5\% vs. $1.5 \%$; O'Neil et al., 2012). Weight loss was accompanied by improvements in glycemic control; however, symptomatic hypoglycemia occurred in $7.4 \%$ of lorcaserin-treated participants. As such, regular monitoring of glucose levels before and during treatment as well as appropriate antidiabetic regimen modifications are recommended. Most common adverse reactions (>5\%) in non-diabetic participants were headache, dizziness, fatigue, nausea, dry mouth, and constipation; in diabetic participants, adverse reactions included hypoglycemia, headache, back pain, cough and fatigue (O'Neil et al., 2012). In contrast to fenfluramine, no increase in rate of cardiac valvulopathy was found after a 2-year lorcaserin treatment (Smith et al., 2010).

Abuse liability was evaluated in a randomized, double-blind placebo-controlled study of recreational polydrug users given acute doses of lorcaserin (20,40 and $60 \mathrm{mg}$ ), zolpidem (15 and $30 \mathrm{mg}$ ), ketamine (100 mg) and placebo (Shram et al., 2011). Abuse liability was evaluated using a visual analog scale for drug liking, which directly correlates with abuse potential (FDA, draft guidance, 2009). While subjective effects of $20 \mathrm{mg}$ lorcaserin were similar to placebo, supratherapeutic lorcaserin doses (40 and $60 \mathrm{mg}$ ) were associated with "dislike" compared with placebo. In contrast to effects produced by lorcaserin, ketamine and zolpidem produced greater "liking" than placebo (Shram et al., 2011). These findings suggest that lorcaserin is well tolerated and lacks abuse liability at therapeutic doses.

Of note, increased serotonin transmission can lead to the serotonin syndrome, which is potentially life-threatening and characterized as a combination of mental status changes, autonomic instability, and neuromuscular hyperactivity (Sternbach, 1991; Brown et al., 1996). Since lorcaserin stimulates the serotonergic system, drug interactions may occur, suggesting that studies are needed which assess the development of the serotonin syndrome in patients using lorcaserin when in combination with other serotonergic agents (Gustafson et al., 2013).

6.1.4. Phentermine and Topiramate (Qsymia®)—In 2012, the FDA approved phentermine and topiramate as a combination therapy for use with a reduced-calorie diet and increased exercise to achieve effective weight loss in obesity (FDA, 2012b). Qsymia ${ }^{\circledR}$ is a fixed dose, controlled-release, combination product containing immediate-release phentermine hydrochloride and extended-release topiramate. Appetite suppressant effect of phentermine, a potent inhibitor of the NE transporter, is thought to be mediated by activation of POMC arcuate nucleus neurons (Fleming et al., 2013). Topiramate (2, 3, 4, 5-bis-O-(1methylethylidene)-beta-D-fructopyranose sulfamate), a GABA agonist, was approved for the treatment of epilepsy (Lee et al., 2003). Patients treated with topiramate exhibited decreased calorie intake and weight loss (Ben-Menachem et al., 2003). Topiramate-induced appetite suppression may be due to modulation of voltage-gated ion channels, increased activity at GABA-A receptors and/or inhibition of AMPA/kainite glutamate receptors (Richard et al., 2000; Bray et al., 2003). Clinical studies demonstrate topiramate's ability to inhibit compulsive food cravings and addictive behavior. Patients with a binge eating disorder receiving topiramate (starting dose of $25 \mathrm{mg} /$ day, titrated over 8-wks to $400 \mathrm{mg} / \mathrm{day}$ ) experienced reductions in binge eating days and episode frequency, body weight and 
compulsive features of binge eating disorder, compared to placebo (McElroy et al., 2007). Ability of Qsymia ${ }^{\circledR}$ to modulate compulsive food-seeking behavior at a clinically relevant dose combination has not been determined. Nevertheless, Qsymia ${ }^{\circledR}$ is the first combination therapy approved for the treatment of obesity that targets both homeostatic (phentermine) and non-homeostatic (topiramate) mechanisms.

The recommended daily dose of Qsymia ${ }^{\circledR}$ contains $7.5 \mathrm{mg}$ of phentermine and $46 \mathrm{mg}$ of topiramate extended-release, although it is available also at a higher dose $(15 \mathrm{mg}$ of phentermine and $92 \mathrm{mg}$ of topiramate extended-release) for selected patients (FDA, 2012b). After one year of treatment with the recommended and highest daily dose of Qsymia ${ }^{\circledR}$, obese patients had a weight loss of $7.8 \%$ and $9.8 \%$, respectively, compared to $1.2 \%$ with placebo (Gadde et al., 2011). Treatment with Qsymia ${ }^{\circledR}$ ameliorated obesity-associated cardio-metabolic conditions, with reductions in high blood pressure, improved total cholesterol and high-density lipoprotein ratios, and decreased glycated hemoglobin levels in overweight and obese participants with type 2 diabetes (Allison et al., 2012; Colman et al., 2012).

Adverse reactions occurring at a rate of $25 \%$ and at least 1.5 times greater than placebo include paresthesia, dizziness, dysgeusia, insomnia, constipation and dry mouth (Garvey et al., 2012). Qsymia ${ }^{\circledR}$ increases heart rate, thereby requiring regular monitoring, especially when initiating or increasing the dose (FDA, 2012b). Prior to treatment, patients should be assessed for depression and suicide ideation since topiramate increases risk of suicidal thoughts (Colman et al., 2012). Qsymia ${ }^{\circledR}$ is contraindicated during pregnancy since topiramate exposure in the first trimester of pregnancy increases risk of cleft lip with or without cleft palate (Margulis et al., 2012). Therefore, Qsymia ${ }^{\circledR}$ is classified as a pregnancy category X drug, due to teratogenic risk (Margulis et al., 2012).

Given structural similarities between phentermine and amphetamine, Qsymia ${ }^{\circledR}$ is a Schedule IV drug under the FDA Controlled Substances Act (Lonneman et al., 2013). Evaluation of phentermine's addictive potential revealed no abuse liability in overweight, obese and weight loss maintenance patients (Hendricks et al., 2014). Amphetamine-like withdrawal was absent upon abrupt treatment cessation at doses much higher than commonly recommended and after treatment durations of up to 21 years (Hendricks et al., 2014).

6.1.5. Naltrexone and Bupropion (Contrave $\left.{ }^{\circledR}\right)$-Naltrexone ((4R, $\left.4 \mathrm{aS}, 7 \mathrm{aR}, 12 \mathrm{bS}\right)-3$ (cyclopropylmethyl)-4a,9-dihydroxy-2,4,5,6,7a,13-hexahydro-1H-4,12methanobenzofuro[3,2-e]isoquinoline-7-one) is a non-selective opioid receptor antagonist used to treat opioid and alcohol dependence (Heal et al., 2012). Buproprion (( \pm )-2-(tertbutylamino)-1-(3-chlorophenyly)propan-1-one) is an inhibitor of DA and NE transporters and is approved to treat depression and nicotine addiction (Dwoskin et al., 2006). In 2014, Contrave ${ }^{\circledR}$, an extended release oral formulation of naltrexone and bupropion, was approved by the FDA as a combination product for adults with a body mass index of 30 and above or with a body mass index of at least 27 with obesity-related comorbidities (FDA, 2014a). Contrave ${ }^{\circledR}$ treatment is initiated with once daily tablet of $8 \mathrm{mg}$ naltrexone and $90 \mathrm{mg}$ bupropion for wk 1 , and escalated to a maintenance 2 tablets twice daily for a total daily dose of $32 \mathrm{mg}$ naltrexone and $360 \mathrm{mg}$ bupropion (FDA, 2014a). 
In a double-blind, placebo-controlled randomized study, obese or overweight participants with dyslipidemia and/or hypertension that received Contrave ${ }^{\circledR}$ (naltrexone $32 \mathrm{mg} /$ day; burpoion $360 \mathrm{mg} /$ day) for $56 \mathrm{wk}$ exhibited greater weight loss (6.4\%) compared to placebo (1.2\%) (Apovian et al., 2013). Participants treated with Contrave ${ }^{\circledR}$ exhibited greater improvement in various cardiometabolic risk markers, participant-reported weight-related quality of life, and control of eating compared to placebo (Apovian et al., 2013). The most common adverse reactions reported in $25 \%$ participants included nausea, constipation, headache, vomiting, dizziness, insomnia, dry mouth and diarrhea. This combination therapy carries a black box warning of increased risk of suicidal behavior and ideation, and neuropsychiatric symptomology (FDA, 2014a). Post-marketing evaluations were required for Contrave ${ }^{\circledR}$ with respect to cardiovascular risks, clinical efficacy and safety in pediatric patients, dosing in patients with renal or hepatic impairment and interactions with other drugs, as well as a juvenile animal toxicity study to evaluate effects on growth, development, and learning and memory (FDA, 2014a).

The precise mechanism of action of Contrave ${ }^{\circledR}$ as an anti-obesity agent is poorly understood. Modulation of both homeostatic (hypothalamic melanocortin system) and nonhomeostatic systems (mesolimbic DA reward system) has been suggested based on evidence that naltrexone $(1 \mu \mathrm{mol} / \mathrm{L})$, bupropion $(10 \mu \mathrm{mol} / \mathrm{L})$ and their combination increase firing frequency of POMC neurons in hypothalamic slices (Cowley et al., 1999; Greenway et al., 2009). The postulated mechanism is that bupropion stimulates hypothalamic POMC neurons releasing a-MSH, which binds to MC4Rs, leading to a decrease in food intake and an increase in energy expenditure. Under physiological conditions, when a-MSH is released, POMC neurons simultaneously release $\beta$-endorphin, a $\mu$-opioid receptor agonist, which inhibits further release of a-MSH, thus activating a negative feedback loop (Kelly et al., 1990; Cowley et al., 2001; Ibrahim et al., 2003). Naltrexone blocks $\mu$-opioid receptors, preventing the $\beta$-endorphin-mediated negative feedback, and the subsequent increase in POMC activity may underlie the weight loss effects of Contrave ${ }^{\circledR}$ (Billes et al., 2014).

Involvement of the DA reward system in mediating anti-obesity effects of Contrave ${ }^{\circledR}$ is suggested by studies measuring food intake in fasted mice following intra-VTA injection of naltrexone $(1 \mu \mathrm{g})$, bupropion $(1 \mu \mathrm{g})$ or the combination (Sinnayah et al., 2007; Billes et al., 2014). The drug combination produced a greater reduction in food intake compared to either drug alone, revealing a synergistic effect on food intake likely involving food reward mechanisms. Contrave ${ }^{\circledR}$ (naltrexone $32 \mathrm{mg} /$ day; bupropion $360 \mathrm{mg}$ /day for $4 \mathrm{wk}$ ) effects on reactivity to food cues was evaluated in an fMRI study conducted in obese humans under a food-deprived condition (Wang et al., 2014). Contrave ${ }^{\circledR}$ attenuated activation in hypothalamus and enhanced activation in regions involved in inhibitory control (anterior cingulate), internal awareness (superior frontal, insula, superior parietal) and memory (hippocampal) in response to food cues. These findings suggest that, in addition to hypothalamic mechanisms, Contrave ${ }^{\circledR}$-induced weight loss also may be due to favorable alterations in cortical reactivity to food cues, memory and self-control. Thus, Contrave ${ }^{\circledR}$ impacts brain regions influencing both homeostatic and non-homeostatic food intake.

6.1.6. Liraglutide (Saxendå)—Liraglutide is a long-acting GLP-1 analog approved for the treatment of type 2 diabetes (Victoza ${ }^{\circledR}$, [produced by recombinant DNA technology 
(rDNA origin)], $1.8 \mathrm{mg}$; SC; FDA, 2010). In 2014, the FDA approved Saxenda ${ }^{\circledR}$ ([rDNA origin], $3 \mathrm{mg}$; SC), for chronic weight management in obese and overweight adults with at least one weight-related comorbidity, such as hypertension, diabetes or dyslipidemia (FDA, 2014b). Saxenda ${ }^{\circledR}$ is not used in combination with any other drug belonging to the GLP-1 analog class, including Victoza ${ }^{\circledR}$ (FDA, 2014b). In a 56-wk, randomized, placebo-controlled study, safety and efficacy of Saxenda ${ }^{\circledR}(3 \mathrm{mg}$; SC) for chronic weight management was evaluated in overweight and obese adults without diabetes (Pi-Sunyer et al., 2015). Participants treated with Saxenda ${ }^{\circledR}(8.1 \%)$ exhibited greater body weight loss compared to placebo $(2.7 \%)$. Saxenda ${ }^{\circledR}$ also reduced cardio-metabolic risk factors, improved fasting and postprandial glycemic variables, $\beta$-cell function and insulin sensitivity, and resulted in a delayed onset of type 2 diabetes. Safety and efficacy of Saxenda ${ }^{\circledR}$ for the treatment of diabetes have not been established.

Adverse reactions reported in $\geq 5 \%$ of patients included nausea, hypoglycemia, diarrhea, constipation, vomiting, headache, decreased appetite, dyspepsia, fatigue, dizziness, abdominal pain and increased lipase activity. Serious adverse events included acute pancreatitis, chest pain and bronchitis. Saxenda ${ }^{\circledR}$ can raise heart rate and should be discontinued in those with a sustained increase in resting heart rate. Saxenda ${ }^{\circledR}$ has a boxed warning stating that thyroid gland tumors (thyroid C-cell tumors) have been observed in rodent studies (FDA, 2014b). Although this effect has not been found in humans, Saxenda ${ }^{\circledR}$ should not be used in patients with a personal or family history of medullary thyroid carcinoma or in patients with multiple endocrine neoplasia syndrome type 2 (a disease which predisposes patients to medullary thyroid carcinoma) (FDA, 2014b). The FDA has required post-marketing evaluations for Saxenda ${ }^{\circledR}$ including case registry of medullary thyroid carcinoma for at least 15 years to identify any increase in incidence, and an evaluation of the potential risk of breast cancer. Also required are clinical trials to evaluate dosing, safety and efficacy in paediatric patients, assessment of potential effects on growth, sexual maturation and central nervous system development and function in immature rats, (FDA, 2014b).

\subsection{Anti-obesity drugs in clinical development}

6.2.1. Tesofensine-Tesofensine ((1R, $2 \mathrm{R}, 3 \mathrm{~S}, 5 \mathrm{~S})-3$-(3, 4-dichlorophenyl)-2(ethoxymethyl)-8-methyl-8-azabicyclo[3.2.1]octane)) is a novel potent, non-selective uptake inhibitor of NE, DA and 5-HT (Astrup et al., 2008b). Tesofensine was developed for the treatment of Alzheimer's and Parkinson's disease, but lacked efficacy (Astrup et al., 2008b). Meta-analysis revealed that tesofensine $(0.125-1.0 \mathrm{mg}$, once daily; oral) produced dosedependent weight loss, and $32 \%$ of obese patients had $25 \%$ weight loss following $14 \mathrm{wk}$ of treatment. Weight loss was accompanied by hypophagia, suggesting an appetite suppressant action. In a 24-wk randomized, double-blind, placebo-controlled Phase II trial conducted in obese participants, tesofensine $(0.25 \mathrm{mg}, 0.5 \mathrm{mg}$ and $1 \mathrm{mg})$ resulted in weight loss of $5 \%$, $9 \%$, and $11 \%$, respectively, compared to placebo (2\%) (Astrup et al., 2008a). After $24 \mathrm{wk}$, heart rate was increased by tesofensine $(0.5 \mathrm{mg}$ and $1 \mathrm{mg})$. An increased incidence $(6 \%)$ of depressed mood was reported. Comprehensive assessment of neuropsychiatric adverse events following tesofensine treatment in obese patients is warranted. 
The mechanism underlying the anti-obesity effects of tesofensine was evaluated in a DIO rat model (Axel et al., 2010). Treatment with tesofensine ( $2 \mathrm{mg} / \mathrm{kg}$, SC) for 16 days suppressed daily food intake (49\%) and produced weight loss (14\%), compared to vehicle. Acute tesofensine $(0.5-3 \mathrm{mg} / \mathrm{kg} ; \mathrm{SC})$ dose-dependently decreased food intake, with an $\mathrm{ED}_{50}$ of 1.3 $\mathrm{mg} / \mathrm{kg}$. Tesofensine-induced hypophagia was inhibited by co-administration of prazosin ( $a 1$ adrenoceptor antagonist, $1 \mathrm{mg} / \mathrm{kg}, \mathrm{SC}$ ) and SCH23390 (D1 receptor antagonist, $0.03 \mathrm{mg} / \mathrm{kg}$, $\mathrm{SC}$ ), and not inhibited by RX821002 ( $a 2$ adrenoceptor antagonist, $0.3 \mathrm{mg} / \mathrm{kg}, \mathrm{SC}$ ), haloperidol (D2 receptor antagonist, $0.03 \mathrm{mg} / \mathrm{kg}$, SC), NGB2904 (D3 receptor antagonist, $0.1 \mathrm{mg} / \mathrm{kg}, \mathrm{SC})$, or ritanserin $\left(5-\mathrm{HT}_{2 \mathrm{~A} / \mathrm{C}}\right.$ receptor antagonist, $\left.0.03 \mathrm{mg} / \mathrm{kg}, \mathrm{SC}\right)$. Thus, $a 1$ and D1 receptors appear to be involved in the anti-obesity effects of tesofensine.

6.2.2. Bupropion and Zonisamide (Empatic)_Weight-loss is a common side-effect of the anti-convulsant drug, zonisamide, and this prompted its evaluation as a treatment for obesity (Gadde et al., 2003). Zonisamide (1, 2-benzoxazol-3-ylmethanesulfonamide) is a potent inhibitor of carbonic anhydrase, which is proposed to contribute to weight-loss (De Simone et al., 2008). Detrimental effects of zonisamide, such as depression and sedation, may be overcome by its combination with bupropion (Ioannides-Demos et al., 2011). A 24wk Phase II clinical trial of the sustained release formulation of bupropion (360 mg)zonisamide $(360 \mathrm{mg}$ ) combination produced greater weight loss $(9.2 \%)$ than bupropion $(6.6 \%)$ or zonisamide (3.6\%) alone or compared to placebo (0.4\%) (Ioannides-Demos et al., 2011). The most common adverse events reported were headache, nausea and insomnia. Phase III clinical trials with the fixed dose combination are underway (George et al., 2014).

6.2.3. Exenatide—A study conducted in overweight women with insulin resistance and polycystic ovary syndrome showed the anti-obesity effects of exenatide, a GLP-1 agonist regulating glucose metabolism and insulin secretion (Kolterman et al., 2003; Elkind-Hirsch et al., 2008). Participants were randomized into three treatment groups: exenatide (10 $\mu \mathrm{g}$ twice a day, SC); metformin, an oral anti-diabetic drug (2000 mg/day); or the combination of metformin $(2000 \mathrm{mg})$ and exenatide $(20 \mu \mathrm{g})$ (Elkind-Hirsch et al., 2008). After $24 \mathrm{wk}$ of treatment, the combination therapy resulted in a mean weight loss of $6 \mathrm{~kg}$, whereas each therapeutic alone produced a $2-3 \mathrm{~kg}$ weight loss. Obese subjects treated with exenatide (10 $\mu \mathrm{g}$ twice daily, SC) for $24 \mathrm{wk}$ also showed greater loss $(5 \mathrm{~kg})$ from baseline relative to placebo (2 kg) (Rosenstock et al., 2010). Moreover, a greater percentage of exenatide-treated subjects experienced $\Sigma 5 \%$ body weight reduction at $24 \mathrm{wk}$ compared with placebo (32 vs. $17 \%$, respectively). A once/wk administration of a long-acting formulation of exenatide ( 0.8 or $2 \mathrm{mg}$, SC, for $15 \mathrm{wk}$ ) was evaluated in a randomized, placebo-controlled Phase II study in participants with type 2 diabetes (Kim et al., 2007). Participants receiving $2 \mathrm{mg}$ exenatide exhibited body weight reductions $(3.8 \mathrm{~kg}$ ), whereas body weight was not changed in participants treated with either $0.8 \mathrm{mg}$ exenatide or placebo. Thus, higher exenatide doses were required for body weight loss. Fasting plasma glucose and $\mathrm{A} 1 \mathrm{C}$ levels were decreased significantly in participants treated with either doses of exenatide, compared to placebo. Exenatide $\left(\right.$ Byetta $\left.{ }^{\circledR}\right)$ was approved by the FDA to improve glycemic control in patients with type 2 diabetes. Further clinical studies investigating the long-term efficacy of exenatide for the treatment of obesity are needed (Dushay et al., 2012). 
6.2.4. Cetilistat-Cetilistat (2-hexadecoxy-6-methyl-3,1-benzoxazin-4-one) is a gastricand pancreatic-lipase inhibitor that is currently undergoing Phase III clinical trials for the treatment of obesity (Charmot, 2012; Jackson et al., 2014). Although the mechanism of action and efficacy of cetilistat is similar to that of orlistat, Phase II clinical trials reveal a superior safety profile for cetilistat (Kopelman et al., 2010). A randomized, placebocontrolled, double-blind study conducted in obese participants with type 2 diabetes revealed that cetilistat ( 80 and $120 \mathrm{mg}$; three/day) or orlistat (120 mg; three time/day) for $12 \mathrm{wk}$, combined with a hypocaloric, moderate fat diet, produced significant reductions in body weight compared to placebo (Kopelman et al., 2010). Increased body weight loss was found in participants receiving cetilistat 80 or $120 \mathrm{mg}(3.9 \mathrm{~kg}$ and $4.3 \mathrm{~kg}$, respectively) or orlistat $120 \mathrm{mg}(3.8 \mathrm{~kg}$ ) compared to placebo $(2.9 \mathrm{~kg})$. Weight loss was accompanied by significant reduction in waist circumference, a risk factor for cardiovascular disease, as well as improved glycemic control in participants treated with both doses of cetilistat and orlistat. The percentage of reported severe gastrointestinal adverse events in cetilistat-treated participants (9-14\% for 40-120 mg) was lower than for orlistat-treated participants (22\%). Also, the proportion of participants with gastrointestinal adverse events leading to discontinuation was lower in the cetilistat groups (2.5-5\%) compared to orlistat (11.6\%). The difference in severity of adverse events between these drugs could be attributed to structural differences between the molecules which may influence the manner in which these they interact with fat micelles in the intestine (Kopelman et al., 2010). Overall, cetilistat appears to be better tolerated than orlistat and associated with greater compliance as a treatment.

6.2.5. Beloranib—Beloranib ([(3R,4S,5S,6R)-5-methoxy-4-[(2R,3R)-2-methyl-3-(3methylbut-2-enyl)oxiran-2-yl]-1-oxaspiro[2.5]octan-6-yl] (E)-3-[4-[2(dimethylamino)ethoxy]phenyl]prop-2-enoate;oxalic acid), a methionine aminopetidase 2 (MetAP2) inhibitor, is an investigational drug candidate for the treatment of obesity. MetAP2, a member of the dimetallohydrolase family, is encoded by the METAP2 gene in humans, and increased expression of this gene is associated with various cancers (Selvakumar et al., 2002). Inhibitors of MetAP2 were developed originally as antiangiogenic agents (Yeh et al., 2006). Interestingly, MetAP2 inhibition also reduces fat biosynthesis and stimulates fat oxidation and lipolysis, suggesting anti-obesity potential.

Beloranib, a synthetic analog of fumagillin, is a potent and selective MetAP2 inhibitor (Sin et al., 1997). Peripheral administration of beloranib for 7 days reduced cumulative food intake and body weight in obese rodent models including, OLETF rats $(1 \mathrm{mg} / \mathrm{kg}$ per day, SC) and mice with lesions in the arcuate nucleus ( $1 \mathrm{mg} / \mathrm{kg}$ per day; SC), compared vehicle control (Kim et al., 2007). Adipocyte, epididymal and mesenteric fat pad size were decreased in beloranib-treated rats. Beloranib is suggested to act in adipose tissue to inhibit formation of new blood vessels and stimulate apoptosis of endothelial cells, thereby inhibiting adipose tissue expansion. Conditioned taste aversion was assessed in beloranibtreated OLETF rats as a potential mechanism underlying decreases in food intake (Kim et al., 2007). Compared to vehicle control, single peripheral injection of the positive control, lithium chloride ( $0.15 \mathrm{M}$; vol was $2 \%$ body weight) and beloranib (1 or $10 \mathrm{mg} / \mathrm{kg}$ ) produced conditioned taste aversion (decreased saccharin solution intake) in OLETF rats. The 
anorexigenic effect of beloranib can be explained partly by the induction of taste aversion. Further studies are needed to elucidate the mechanistic effects of beloranib on appetite.

A proof of concept clinical trial was conducted in obese women to evaluate the safety, weight loss and cardio-metabolic risk factors of beloranib in the absence of dietary intervention or exercise (Hughes et al., 2013). In a double-blind, placebo-controlled study, obese women were randomized to intravenous beloranib $(0.1,0.3$, or $0.9 \mathrm{mg} / \mathrm{m} 2)$ or placebo twice/wk for $4 \mathrm{wk}$. Beloranib $(0.3$ and $0.9 \mathrm{mg} / \mathrm{m} 2)$ resulted in median body weight loss of $3.5 \%$ at the end of $4 \mathrm{wk}$, compared to $0.6 \%$ following placebo. Beloranib $\left(0.9 \mathrm{mg} / \mathrm{m}^{2}\right)$ also produced a significant reduction in triglycerides and low-density lipoprotein cholesterol, Creactive protein (marker of inflammation) and hunger, evaluated using a visual analog scale. The most frequent adverse effects of mild or moderate intensity included headache, infusion site injury, nausea and diarrhea; however, no serious adverse events were found.

A follow up, Phase II double-blind, randomized, placebo-controlled study investigated the efficacy, safety and tolerability of a beloranib suspension $(0.6,1.2$ and $2.4 \mathrm{mg}, \mathrm{SC})$ in obese women for $12 \mathrm{wk}$ (Kim et al., 2015). At wk 12, beloranib resulted in dose-dependent weight loss of 5-10\% compared to $0.3 \%$ with placebo. Beloranib-induced weight loss was accompanied by reductions in waist circumference and body fat mass. Reduced caloric intake likely contributed to weight loss, as the beloranib-treated participants reported a marked reduction in hunger. The highest dose of beloranib resulted in significant improvements in mean total cholesterol, low-density lipoprotein and high-density lipoprotein cholesterol, triglyceride levels and systolic blood pressure, compared with placebo. No serious adverse events were reported. The most common side-effects included sleep disturbance and gastrointestinal disorders, which were generally mild to moderate in the high dose group. Long-term studies are needed in a larger and diverse patient population, which includes participants with obesity-related comorbidities, to confirm the safety, efficacy and tolerability of beloranib for weight loss and improvements in cardio-metabolic risk factors.

6.2.6. $\boldsymbol{R M}$-493-RM-493 is a selective, novel, MC4R peptide agonist, which has been evaluated in a non-human primate model of DIO (Kievit et al., 2013). Chronic SC infusion of RM-493 for 8 wk in obese rhesus macaques decreased food intake, increased energy expenditure, reduced body weight, and improved glucose tolerance without causing adverse cardiovascular effects. A recent randomized, double-blind, placebo-controlled, crossover study examined the effects of RM-493 on resting energy expenditure in healthy obese adults (Chen et al., 2015). RM-493 (1 mg/24 h) or placebo was given by continuous SC infusion over $72 \mathrm{hr}$. Participants received a weight-maintenance diet (50\% carbohydrate, $30 \%$ fat and $20 \%$ protein) and performed $30 \mathrm{~min}$ of standardized exercise daily. Compared to placebo, RM-493 resulted in a 6.4\% increase in resting energy expenditure and increased plasma GLP-1 and PYY levels. No adverse effect on heart rate or blood pressure was found. Surprisingly, RM-493 treatment was associated with increased plasma glucose, insulin and C-protein levels. Long-term clinical studies are needed to elucidate the effects of RM-493 on insulin sensitivity. RM-493 is undergoing evaluation in a parallel mechanistic Phase Ib study in obese participants to determine whether RM-493-induced weight loss is caused by decreased appetite, increased metabolism or both (Jackson et al., 2014). Long-term studies 
in a diverse and larger population are needed to obtain a better understanding of the safety, efficacy and tolerability of RM-493 for the treatment of obesity.

6.2.7. KD026—KD026 (1-[[3-methoxy-2-[4-(trifluoromethyl)phenyl]benzoyl]amino]-3,4dihydro-1H-isoquinoline-2-carboxylic acid) is a novel, nonsystemically available intestinal microsomal transfer protein inhibitor under clinical investigation for the treatment of obesity (Kim et al., 2011; Jackson et al., 2014). Microsomal transfer protein is a heteromeric protein involved in the synthesis of chylomicrons and apolipoprotein B-containing lipoproteins, impacting the transport of lipids and cholesterol from the intestine and liver to tissues (Cuchel et al., 2013). First-generation microsomal transfer protein inhibitors were designed to inhibit hepatic proteins and provide a novel treatment for dyslipidemia (Roevens et al., 1999). While potent inhibitors of hepatic microsomal transfer protein were efficacious in reducing low-density lipoprotein-cholesterol, these inhibitors resulted in elevation of liver enzymes and hepatic steatosis in animals and humans (Roevens et al., 1999; Gruetzmann et al., 2000).

Microsomal transfer protein is highly expressed in the enterocytes lining the lumen of the jejunum and is critical to the production of chylomicrons assembled from lipid or cholesterol, and their transfer into the systemic circulation (Gordon and Jamil, 2000; Hussain and Bakillah, 2008). KD026 was designed to selectively inhibit microsomal transfer protein $\left(\mathrm{IC}_{50}=\sim 8 \mathrm{nM}\right)$ in the intestinal lining without exerting an impact on the liver (Kim et al., 2011). KD026 (30 mg/kg; oral) reduced postprandial lipids by $>50 \%$ with an $\mathrm{ED}_{50}$ value of $\sim 7 \mathrm{mg} / \mathrm{kg}$ in rats (Kim et al., 2011). Chronic effects of KD026 were evaluated in apolipoprotein-E deficient mice, a dyslipidemic animal model, in which the severity of hyperlipidemia is exacerbated by high-fat diet. Apolipoprotein-E deficient mice maintained on a high-fat diet and KD026 (19 mg/kg/day or $67 \mathrm{mg} / \mathrm{kg} / \mathrm{day}$; oral) for $10 \mathrm{wk}$ exhibited decreased levels of plasma triglycerides, total cholesterol and low-density lipoprotein cholesterol and increased levels of high-density lipoprotein cholesterol, compared to vehicle and high-fat diet (Kim et al., 2011). Moreover, KD026 (19 or $67 \mathrm{mg} / \mathrm{kg} /$ day) decreased body weight gain in apolipoprotein-E deficient mice over the course of treatment and reduced visceral fat and liver weight. Enterocytic selectivity of KD026 was supported by lack of elevation of liver transaminases even at the highest dose tested. Sub-chronic studies revealed that KD026 was devoid of any toxic effects when administered to rats for 90 days at a dose of $1000 \mathrm{mg} / \mathrm{kg}$ per day (Kim et al., 2011). Overall, inhibition of enterocytic microsomal transfer protein appears to be a promising peripheral target for the treatment of obesity.

6.2.8. Remogliflozin etabonate-The bulk of the filtrated glucose in kidney tubules is reabsorbed mainly by the low-affinity sodium-glucose cotransporter 2 (Kanai et al., 1994). Sodium-glucose cotransporter 2 inhibitors block the re-absorption of glucose by the kidney, thereby enhancing glucose excretion through the urine and resulting in a reduction in fasting plasma glucose levels and haemoglobin A1c levels. Remogliflozin etabonate (ethyl [(2R,3S, 4S,5R,6S)-3,4,5-trihydroxy-6-[5-methyl-1-propan-2-yl-4-[(4-propan-2-

yloxyphenyl)methyl]pyrazol-3-yl]oxyoxan-2-yl]methyl carbonate) is a prodrug of remogliflozin, a selective inhibitor of the sodium-glucose cotransporter 2 (Fujimori et al., 2008). In both mice and rats, remogliflozin etabonate (3-30 and 1-10 mg/kg, respectively, 
oral) increased urinary glucose excretion in a dose-dependent manner (Fujimori et al., 2008). In normal rats, remogliflozin etabonate $(1-10 \mathrm{mg} / \mathrm{kg}$ ) inhibited increases in plasma glucose after glucose loading without stimulating insulin secretion (Fujimori et al., 2008). In agreement, a single dose of remogliflozin etabonate $(150 \mathrm{mg}$ or $500 \mathrm{mg}$ ) was shown to increase urine glucose excretion and lower plasma glucose in human participants with type 2 diabetes mellitus (Kapur et al., 2013). Remogliflozin etabonate is being evaluated currently in obese patients as a potential weight loss therapy (Jackson et al., 2014).

In a 12-wk Phase $2 b$ clinical trial, canagliflozin ( $2 \mathrm{~S}, 3 \mathrm{R}, 4 \mathrm{R}, 5 \mathrm{~S}, 6 \mathrm{R})-2-[3-[[5-(4-$ fluorophenyl)thiophen-2-yl]methyl]-4-methylphenyl]-6-(hydroxymethyl)oxane-3,4,5-triol; 50, 100 and $300 \mathrm{mg}$ ), another sodium-glucose cotransporter 2 inhibitor, decreased body weight and increased urinary glucose excretion in a dose-dependent manner in overweight and obese participants without diabetes mellitus compared with placebo (Bays et al., 2014). A limitation of this trial was the $25 \%$ participant discontinuation rate. Canagliflozin was associated with higher rates of genital mycotic infections in women. Long-term safety and efficacy studies of are needed in obese individuals with metabolic abnormalities.

\subsection{Potential anti-obesity candidates and targets based on preclinical and preliminary human studies}

6.3.1 RM-493 and Liraglutide-Efficacy of adjunctive RM-493 and liraglutide treatment was evaluated in an animal model of DIO (Clemmensen et al., 2015). DIO mice were treated with RM-493 (3.6 $\mu \mathrm{mol} / \mathrm{kg} / \mathrm{day})$, liraglutide ( $10 \mathrm{nmol} / \mathrm{kg} / \mathrm{day})$, the combination of liraglutide and RM-493 or vehicle for 5 consecutive days. Treatment with either monotherapy decreased body weight relative to vehicle. While no differences in weight reduction were found between mice treated with liraglutide and RM-493 alone, co-treatment resulted in greater weight loss relative to either monotherapy. Moreover, the combination therapy decreased caloric intake, improved glycemic control and cholesterol metabolism beyond that achieved with either monotherapy. Acute ( $48 \mathrm{~h}$ ) treatment with liraglutide decreased (83\%) hypothalamic GLP-1 receptor mRNA levels, compared to vehicle. The liraglutide-mediated down-regulation of GLP-1 receptor mRNA was prevented when RM-493 was coadministered with liraglutide, suggesting the possible role for RM-493 in maintaining GLP-1 receptor expression. Future studies to define the molecular mechanisms underlying the combinatorial benefits of MC4R and GLP-1 receptor agonism in energy metabolism are warranted. These findings suggest therapeutic potential of RM-493 and liraglutide combination for the treatment of obesity and diabetes.

6.3.2. Pramlintide and Metreleptin—Leptin plays a pivotal role in the physiological regulation of body weight. However, as a result of leptin resistance in obesity, development of recombinant leptin as a stand-alone obesity treatment has proven unsuccessful. The ability of amylin to restore leptin responsiveness in DIO rats suggests the therapeutic potential of a leptin and amylin combination for the treatment of obesity (Roth et al., 2008). Concurrent peripheral administration of amylin (100 $\mu \mathrm{g} / \mathrm{kg} /$ day for 14 days, SC) and leptin $(500 \mu \mathrm{g} / \mathrm{kg} /$ day for 14 days, SC) produces synergistic, fat-specific weight loss in leptinresistant, DIO rats compared to vehicle control (Roth et al., 2008). In a 24-wk randomized, double-blind, proof-of-concept study in overweight/obese individuals, co-administration of 
the amylin analog, pramlintide ( $180 \mu \mathrm{g}$ twice daily for $2 \mathrm{wk}$ and $360 \mu \mathrm{g}$ twice daily thereafter, SC) and recombinant human leptin, metreleptin (5 mg, twice daily, SC) resulted in a $12.7 \%$ mean weight loss, which was significantly more than with either treatment alone (8.4\% for pramlintide and $8.2 \%$ for metreleptin) (Ravussin et al., 2009). The most common adverse events reported in the combined treatment group were reactions at the injection site $(58.9 \%)$ and nausea (12.5\%). In most patients, nausea subsided after $5 \mathrm{wk}$ of treatment. Together, these findings provide both preclinical and clinical evidence that integrated neurohormonal approaches may result in greater weight loss. Further clinical evaluation of the safety and efficacy of pramlintide-metreleptin for the treatment of obesity are needed.

6.3.3. Glucagon and GLP-1 receptor agonism-Glucagon and GLP-1 are pancreatic and intestinal hormones, respectively, derived from the same proglucagon peptide, but with divergent roles in metabolism (Tan et al., 2013). Glucagon is a 29 amino acid peptide hormone that is derived from pancreatic islet a cells as well as brainstem (Drucker and Asa, 1988; Sadry and Drucker, 2013). Under conditions of hypoglycemia, glucagon elevates blood glucose by promoting glycogenolysis and gluconeogenesis, as well as hepatic fatty acid $\beta$-oxidation and ketogenesis (MacDonald et al., 2007; Cryer, 2012). Peripheral administration of glucagon decreases food intake in rats and humans (Penick and Hinkle, 1961; Martin and Novin, 1977). Moreover, glucagon infusion increases oxygen consumption in rats and increases resting energy expenditure in humans (Davidson et al., 1957; Nair, 1987). GLP-1 is an incretin hormone released postprandially that enhances $\beta$-cell insulin response, inhibits gastric emptying, and suppresses appetite (Drucker, 2007). The role of glucagon and GLP-1 receptor activation in the regulation of satiety and metabolism suggests that co-agonists exhibiting optimal ratios of GLP-1 and glucagon agonism might produce additive or synergistic effects on weight loss (Sadry and Drucker, 2013). While activation of glucagon receptors increases glucose production posing a hyperglycemic risk, simultaneous activation of GLP-1 receptors counteracts this effect (Pocai, 2014).

Oxyntomodulin, a 37-amino acid peptide hormone secreted from gut along with GLP-1 following nutrient ingestion, is produced in brainstem and transported distally to hypothalamus (Blache et al., 1988; Drucker and Asa, 1988; Kieffer and Habener, 1999). Oxyntomodulin serves as an agonist at both GLP-1 and glucagon receptors; however, with 10- to 100-fold reduced potency compared with the cognate agonists GLP-1 and glucagon, respectively (Gros et al., 1993; Mayo et al., 2003; Baggio et., 2004). A randomized, controlled, double-blind, crossover study investigated the effect of oxyntomodulin on body weight in obese, non-diabetic participants (Wynne et al., 2005). Subcutaneous selfadministration of pre-prandial oxyntomodulin ( $400 \mathrm{nmol} ; 3$ times/day for $4 \mathrm{wk}$ ) decreased body weight and plasma leptin levels and increased plasma adiponectin levels, compared to control. Moreover, pre-prandial oxyntomodulin ( $400 \mathrm{nmol} ; 3$ times/day for 4 days) increased energy expenditure and decreased energy intake compared to control. Further mechanistic understanding and long-term studies of oxyntomodulin in individuals with diabetes are needed.

The anti-obesity effects of a long-acting, protease-resistant, dual GLP-1/glucagon receptor agonist was compared to that of a long-acting GLP-1 receptor selective agonist in a mouse DIO model (Pocai et al., 2009). Repeated treatment with the dual agonist ( $1.9 \mu \mathrm{mol} / \mathrm{kg}$ every 
other day for $2 \mathrm{wk}$; SC) resulted in greater weight loss and lipid lowering in DIO mice, compared to mice treated with the GLP-1 receptor selective agonist $(1.9 \mu \mathrm{mol} / \mathrm{kg}$ every other day for $2 \mathrm{wk}$; SC). Moreover, compared to vehicle-treated DIO mice, plasma glucose levels were normalized and glucose tolerance was improved upon treatment with either the dual agonist or selective GLP-1 receptor agonist. Improvements in plasma metabolic parameters including insulin, leptin, and adiponectin, increased fatty acid oxidation and reduced hepatic steatosis were more pronounced upon treatment with the dual agonist than with the selective GLP-1 receptor agonist alone in DIO mice. Weight loss was observed with dual agonist treatment in both GLP-1 receptor knockout and glucagon receptor knockout mice; however, efficacy was reduced compared with body weight effects in weight-matched wild-type mice (Pocai et al., 2009). These results suggest that the anti-obesity effects of the dual agonist requires activation of both GLP-1 and glucagon receptors.

In a randomized, double-blinded crossover study, overweight or obese participants without diabetes were given GLP-1 (0.8 pmol/ $/ \mathrm{kg} / \mathrm{min}, \mathrm{IV})$, glucagon $(50 \mathrm{ng} / \mathrm{kg} / \mathrm{min}, \mathrm{IV})$ or a combination of both GLP-1 and glucagon (Tan et al., 2013). Compared to control, resting energy expenditure was increased upon treatment with glucagon alone and the combination of glucagon and GLP-1, but was not altered by GLP-1 infusion. Although glucagon infusion was accompanied by a rise in plasma glucose levels, addition of GLP-1 ameliorated this effect. Together, drugs with glucagon and GLP-1 agonist activity may be beneficial for the treatment of obesity and type 2 diabetes.

6.3.4. Growth hormone-Growth hormone, or somatotropin, is best known for its action on linear growth such that deficiency leads to dwarfism and excess leads to gigantism (Berryman et al., 2013). In addition to normal growth, growth hormone stimulates the production of insulin-like growth factor-1 in most tissues, which collectively regulate fat, protein and glucose metabolism. Clinical features of adults with severe growth hormone deficiency as a result of hypothalamic-pituitary disease include increased total body adipose tissue mass, abdominal obesity, atherogenic lipid profile, premature atherosclerosis, reduced exercise capacity and insulin resistance (Johannsson, 2007). In obesity, both spontaneous and stimulated growth hormone secretion are blunted (Williams et al., 1984; Veldhuis et al., 1991). An inverse association exists between growth hormone secretion and the amount of ectopic fat deposition in the muscle and liver (Clasey et al 2001; Bredella et al., 2009). Moreover, the metabolic clearance rate of growth hormone is accelerated in individuals with obesity (Veldhuis et al., 1991). Overall, there appears to be dysregulated growth hormone function associated with obesity.

Growth hormone treatment $(0.5$ and $5 \mu \mathrm{g} / \mathrm{g} /$ day for $6 \mathrm{wk}$; SC) results in fat mass reduction and increase in lean mass in DIO mice, despite free access to a high-fat diet during the treatment period, compared to vehicle-control mice maintained on a low-fat diet (List et al., 2009). Also, growth hormone reduced liver triacylglycerol and fasting blood glucose and improved glucose tolerance in DIO mice to levels in low-fat fed controls. Liver, skeletal muscle and adipose tissue develop insulin resistance in response to acute growth hormone administration in humans (Jessen et al., 2005; Nielsen et al., 2008). Mechanisms responsible for growth hormone-induced insulin resistance remain unclear. Insulin resistance may be secondary to stimulation of lipolysis and subsequent free fatty acid release, which results in 
competition between glucose and free fatty acid as a substrate for energy production (Møller, N. \& Jørgensen, 2009).

A 12-wk, placebo-controlled trial of growth hormone treatment combined with caloric restriction was conducted in obese participants with type 2 diabetes (Nam et al., 2001). Growth hormone treatment $(0.15 \mathrm{IU} / \mathrm{kg}$ for $12 \mathrm{wk})$ reduced visceral adipose tissue mass and levels of low density lipoprotein cholesterol and improved insulin sensitivity measured by euglycaemic hyperinsulinaemic clamp. In a double-blind, placebo-controlled crossover design, growth hormone administration $(0.03 \mathrm{mg} / \mathrm{kg}$ for $5 \mathrm{wk})$ in obese women resulted in increased resting energy expenditure, decreased respiratory exchange ratio and increased rate of lipid oxidation (Jørgensen et al., 1994). Alterations in body composition and an increase in energy expenditure might counteract the direct effects of growth hormone on insulin resistance (Berryman et al., 2013). Overall, both animal and human studies demonstrate the therapeutic potential of growth hormone in the treatment of obesity.

6.3.5. Bile acid sensors: farnesoid $X$ receptor (FXR) and membrane bound bile acid receptor (GPR131)-Bile acids are synthesized in the liver from cholesterol where they are conjugated to glycine (human) or taurine (mouse), and metabolized to secondary bile acids in the gut by the microbiota (Midtvedt, 1974; Sayin et al., 2013). Bile acids serve as signaling molecules that act through the nuclear receptor, FXR, and membrane-bound GPCR, GPR131 or TGR5, to regulate lipoprotein and glucose metabolism (Makishima et al., 1999; Sinal et al., 2000; Maruyama et al., 2002; Kawamata et al., 2003). In addition to their role in lipid emulsification and absorption of fat-soluble vitamins, emerging evidence demonstrates the role of bile acids and their receptors in the regulation of energy balance (Maruyama et al., 2006; Penney et al., 2015).

FXR is a ligand-activated transcriptional factor expressed in diverse peripheral tissues including the adrenal gland, kidney, stomach, duodenum, jejunum, ileum, colon, gall bladder, liver and macrophages, as well as in white and brown adipose (Forman et al., 1995). The complex role of FXR in metabolic homeostasis is evident from studies conducted in whole body and liver-specific FXR null mice. During maintenance on a standard chow diet, FXR null mice exhibit elevated levels of serum bile acid, cholesterol, and triglycerides, increased hepatic cholesterol and triglycerides, a proatherogenic serum lipoprotein profile, and reduced fecal bile acid excretion, compared to wild-type mice (Sinal et al., 2000). This suggests that FXR is critical for bile acid and lipid homeostasis. In order to investigate the role of FXR in the adaptation to obesity and its metabolic complications, the impact of FXR deficiency was studied in rodent models of obesity (Prawitt et al., 2011). FXR deficiency attenuated body weight gain in ob/ob mice and mice maintained on a high-fat diet for $20 \mathrm{wk}$ by reducing adipose tissue mass, compared to wild-type controls. FXR deficiency improved glucose homeostasis in both rodent models of obesity as a result of enhanced glucose clearance and adipose tissue insulin sensitivity. These metabolic improvements were not mediated by hepatic FXR, since liver-specific FXR deficient mice were not protected from DIO and insulin resistance. Overall, in contrast with lean mice, FXR deficiency in obesity improves energy and glucose homeostasis. 
In response to a meal, bile acids are secreted into intestine from the gall bladder and acutely activate intestinal FXR (Fang et al., 2015). In order to study the specific role of intestinal FXR activity in the regulation of energy metabolism, DIO mice were orally administered a synthetic FXR agonist, fexaramine (methyl (E)-3-[3-[cyclohexanecarbonyl-[[4-[4(dimethylamino)phenyl]phenyl]methyl]amino]phenyl] prop-2-enoate; $100 \mathrm{mg} / \mathrm{kg}$ daily for 5 wk), (Fang et al., 2015). Compared to vehicle control, fexaramine resulted in a significant reduction in body weight gain that was attributed to reduced overall fat mass including subcutaneous and visceral adipose depots. Consistent with reduced adiposity, fexaraminetreated mice showed significant metabolic improvements including reduced glucose, insulin, leptin, cholesterol and resistin levels. Furthermore, fexaramine resulted in improvements in glucose tolerance, insulin sensitivity, reduced adipose tissue inflammation and enhanced thermogenesis and browning of white adipose tissue. These results suggest that FXR agonism is a potential approach to the treatment of obesity and insulin resistance.

Previous studies support the beneficial effects of whole body FXR deficiency in rodent models of obesity (Prawitt et al., 2011). Mice with intestine-specific FXR deletion are resistant to DIO ( $\mathrm{Li}$ et al., 2013). FXR signaling is activated in distal ileum biopsies from obese humans compared to lean humans, as evidenced by increase in mRNAs encoded by FXR and FXR target genes (Jiang et al, 2015). Based on these results, glycine- $\beta$-muricholic acid, an orally available, intestine-specific FXR inhibitor, was used to examine whether selective inhibition of intestinal FXR ameliorates obesity and associated metabolic abnormalities (Jiang et al., 2015). Compared to vehicle control, glycine- $\beta$-muricholic acid $(10 \mathrm{mg} / \mathrm{kg}$ daily for 5-6 wk) increased energy expenditure, decreased body weight, fat mass, fat/lean mass ratio, insulin resistance and hepatic steaosis when orally administered to leptin-receptor deficient mice and DIO mice. Inhibition of FXR resulted in decreased expression of ceramide synthesis-related genes suggesting that decreases in intestinalderived ceramides likely mediate the resolution of DIO and hepatic steatosis. These results demonstrate that specific inhibition of intestinal FXR may be a therapeutic strategy for the treatment of obesity and metabolic disorders. However, these findings are in contrast to prior evidence suggesting a role of gut-restricted FXR agonism in ameliorating DIO and associated metabolic dysfunction (Fang et al., 2015). Studies are needed regarding the mechanistic role of FXR in the pathophysiology of obesity.

The gut microbial community (microbiota) is considered an environmental factor that modulates host metabolism and contributes to the development of obesity and metabolic diseases. Gut microbiota is essential for processing dietary polysaccharides (Backhed et al., 2004). Conventionalization of adult germ-free mice with normal microbiota harvested from the distal intestine (cecum) of conventionally raised animals produced a $60 \%$ increase in body fat content, de novo hepatic lipogenesis and insulin resistance within 14 days (Backhed et al., 2004). Also, faecal microbiota transplants from lean glucose-sensitive human donors were shown to improve insulin sensitivity in humans with the metabolic syndrome (Vrieze et al., 2012). These results identify altered gut microbiota as a contributing factor to the pathophysiology of obesity.

Recent evidence suggests that microbiota-mediated changes in bile acid profiles and signaling through FXR contribute to impaired host metabolism (Parseus et al., 2016). Germ- 
free and conventionally raised wild-type and FXR deficient mice were fed a high-fat diet for $10 \mathrm{wk}$. Gut microbiota promoted weight gain, adipose tissue inflammation, beta-cell mass and hepatic steatosis in an FXR-dependent manner. Bile acid profiles and composition of faecal microbiota differed between FXR deficient and wild-type mice suggesting that gut microbiota promotes DIO through effects on the bile acid profile and altered FXR signaling. Further understanding of gut microbiota-mediated modulation of bile acids and signaling through FXR may provide novel therapeutic targets for obesity and metabolic diseases.

Another bile acid receptor that is expressed in diverse tissues and is suggested to regulate energy homeostasis is TGR5 (Hodge and Nunez, 2016). In enteroendocrine cells, TGR5 stimulates the release of both GLP-1 and PYY thereby maintaining glucose homeostasis and decreasing food intake (Thomas et al., 2009; Bala et al., 2014). In muscle and brown adipose tissue, TGR5 regulates energy expenditure through activation of thyroid hormone-activating enzyme, type 2 iodothyronine deiodinase (Eggink et al., 2014). TGR5 is also located in neurons of the enteric nervous system, where they influence gut motility (Poole et al., 2010), and in neurons and astrocytes in the central nervous system, where its function is unknown. Further animal studies are needed to investigate the therapeutic potential of TGR5 for the treatment of obesity and type 2 diabetes.

\section{Challenges for anti-obesity drug development}

Considering the numerous withdrawals of anti-obesity drugs from the market due to untoward effects, benchmarks for safety and efficacy for anti-obesity drugs are heavily scrutinized. With respect to safety, the FDA requires that anti-obesity drugs should not adversely affect cardiovascular function. Cardiac valvulopathy is highlighted as a serious adverse effect in the Unites States as a result of past experience with both dexfenfluramine and the fenfluramine/phentermine (fen/phen) combination (Heal et al., 2009). The majority of currently approved anti-obesity drugs and those in clinical development have effects mediated by central mechanisms (homeostatic and/or reward systems). For centrally-acting drug candidates, psychiatric adverse events, abuse liability, and side-effects including dependence and withdrawal are potential safety issues that require careful investigation. Preclinical research that offers predictive validity with respect to the assessment of abuse potential and other neuropsychiatric complications are beneficial particulary in early stages of drug development.

FDA criteria for weight reduction after 1 year of treatment with an anti-obesity drug is defined as $\geq 5 \%$ body weight compared to placebo control (FDA draft guidance, 2007; Heal et al., 2009). The FDA also states that in order to ensure that drug-induced weight loss is due to primarily a reduction in fat content, not lean-body mass, a representative sample of subjects should be assessed for body composition by dual energy x-ray absorptiometry or using a suitable alternative at baseline and follow-up (FDA draft guidance, 2007). In addition to weight reduction, FDA regulations stipulate that an effective weight-management product should provide improvements in blood pressure, lipids, glycemia or other metabolic complications. Thus, clinical trials of candidate anti-obesity medications need to include assessments of efficacy for common weight-related comorbidities. 
Tolerance develops to the anti-obesity effects of pharmacological monotherapies as a result of counter-regulation. As such, drug discovery has shifted to a multi-target approach with an overall objective of enhancing therapeutic outcome. An additional layer of complexity in clinical assessment arises regarding the safety and efficacy of fixed-dose combination products. With respect to efficacy, therapeutic effects of the combination product must be superior to placebo and to the relevant doses of the individual drugs in the combination product (Nathan et al., 2011). While a combination strategy may facilitate therapeutic efficacy, the incidence of adverse effects may increase. Nevertheless, if adverse events are not severe and if a synergistic effect is found with the combination product, then doses of the individual drugs may be reduced to overcome safety issue.

The American Psychiatric Association's Diagnostic and Statistical Manual of Mental Disorders list the diagnostic criteria for behavioral disorders including depression, substance abuse and other personality disorders. A striking similarity is noted between behaviors that are considered criteria for a diagnosis of substance abuse and for obesity, including a persisent desire for food and maintenance of overeating despite knowledge of adverse physical and psychological consequences caused by excessive food consumption (Volkow and O'Brien, 2007). Obesity may be considered a "food addiction", although further examination of this concept is needed. The Yale Food Addiction Scale was developed to operationalize the concept of "food addiction" (Gearhardt et al., 2009). This scale allows application of substance dependence diagnostic criteria to eating behavior and provides two scoring options; one that measures food addiction "symptoms" and another option that provides a food addiction "diagnosis" based upon the substance dependence diagnosis (Gearhardt et al., 2012). Using the Yale Food Addiction Scale, the nature of "food addiction" was examined in treatment-seeking obese participants with binge-eating disorder (Gearhardt et al., 2012). Classification of "food addiction" was met by $57 \%$ of participants with binge eating disorder. However, it should be noted that the proportion of binge eaters in the obese population is a small minority. In another study, addictive-like eating patterns in children, as measured by the Yale Food Addiction Scale, were related to elevated BMI and a greater tendency to overeat in response to emotional stimuli (Gearhardt et al., 2013). Overall, the issue of "food addiction" as a cause of or result of obesity remains controversial and requires further investigation.

Common brain substrates regulate the hedonic properties of palatable food and addictive drugs, suggesting that excessive consumption of food or drugs of abuse produces similar neuroadaptive responses in brain reward circuitries (Kenny, 2011). As such, behavioral paradigms that have been employed extensively to assess motivational aspects of drug addiction and relapse may be beneficial in the assessment of anti-obesity drugs. For example, 5-HT neurotransmission via $5-\mathrm{HT}_{2 \mathrm{~A}}$ and $5-\mathrm{HT}_{2 \mathrm{C}}$ receptors have been implicated in mediating cocaine abuse as well as impulsive behaviors that contribute to the development of cocaine addiction and relapse in humans (Howell and Cunningham, 2015). However, a gap in our knowledge exists with respect to the ability of centrally-acting weight loss drugs to inhibit relapse of palatable food-seeking behavior. Clinical trials have not determined whether monoamine-based anti-obesity medications that lack abuse liability have efficacy in reducing craving and preventing relapse of palatable food consumption. Thus, in addition to the evaluation of cardio-metabolic improvements, assessment of relapse to palatable food 
consumption and of abuse potential for alternate reinforcers (e.g., abused drugs) would enhance evaluations of anti-obesity treatment.

\section{Conclusions}

Research over the past two decades has advanced substantially our knowledge of central and peripheral mechanisms underlying energy balance, making these mechanisms attractive targets for anti-obesity therapeutics. In addition to homeostatic systems, targeting nonhomeostatic mechanisms may prove beneficial in the amelioration of maladaptive rewarddriven palatable food consumption. Given that multiple central and peripheral mechanisms underlie the development and maintenance of obesity, combination therapies may exert greater and longer-term weight loss compared to monotherapies. Currently two combination products have been approved by the FDA for the treatment of obesity. There are several potential combination therapy approaches that appear beneficial. However, additional longterm studies are needed, especially for centrally acting anti-obesity drugs, to evaluate potential neuropsychiatric adverse effects and the benefit to risk ratio. Future studies focusing on not only central, but also peripheral targets governing energy expenditure, may offer novel treatment strategies.

\section{ACKNOWLEDGEMENTS}

The authors acknowledge support from NIH grants DA05312, HL73085, RR021954, UL1TR000117, UL1TR001998 and a Predoctoral Fellowship from the American Heart Association, AHA 715489B.

\section{ABBREVIATIONS}

$\begin{array}{ll}\text { AgRP } & \text { agouti-related protein } \\ \text { a-MSH } & \text { alpha-melanocyte stimulating hormone } \\ \text { 2-AG } & \text { 2-arachidonoylglycerol } \\ \text { BDNF } & \text { brain derived neurotrophic factor } \\ \text { BOLD } & \text { blood-oxygen level dependent } \\ \text { CB1 } & \text { cannabinoid-1 } \\ \text { CB2 } & \text { cannabinoid-2 } \\ \text { CART } & \text { cocaine and amphetamine regulated transcript } \\ \text { CCK } & \text { cholecystokinin } \\ \text { CPP } & \text { conditioned place preference } \\ \text { DIO } & \text { diet-induced obesity } \\ \text { DA } & \text { dopamine } \\ \text { DAT } & \text { dopamine transporter }\end{array}$




\begin{tabular}{|c|c|}
\hline FXR & farnesoid $X$ receptor \\
\hline fMRI & functional magnetic resonance imaging \\
\hline GLP-1 & glucagon-like peptide-1 \\
\hline GHSR1A & growth hormone secretagogue receptor $1 \mathrm{~A}$ \\
\hline GABA & $\gamma$-aminobutyric acid \\
\hline GPCRs & G-protein coupled receptors \\
\hline ICV & intracerebroventricular \\
\hline IP & intraperitoneal \\
\hline МСH & melanin-concentrating hormone \\
\hline MC4R & melanocortin-4 receptor \\
\hline MetAP2 & methionine aminopetidase 2 \\
\hline NE & norepinephrine \\
\hline NPY & neuropeptide $\mathrm{Y}$ \\
\hline NTS & nucleus tractus solitarius \\
\hline OEA & oleoylethanolamide \\
\hline OLETF & Otsuka Long Evans Tokushima Fatty \\
\hline POMC & pro-opiomelanocortin \\
\hline $\mathbf{P R}$ & progressive ratio \\
\hline pSTAT3 & phosphorylated signal transducer and activator of transcription 3 \\
\hline PYY & peptide YY \\
\hline 5-HT & serotonin \\
\hline SC & subcutaneous \\
\hline VTA & ventral tegmental area \\
\hline
\end{tabular}

\section{REFERENCES}

Abdeen G, le Roux CW. Mechanism underlying the weight loss and complications of Roux-en-Y gastric bypass. Review. Obes Surg. 2015 [Epub ahead of print].

Abizaid A, Liu ZW, Andrews ZB, Shanabrough M, Borok E, Elsworth JD, et al. Ghrelin modulates the activity and synaptic input organization of midbrain dopamine neurons while promoting appetite. $\mathrm{J}$ Clin Invest. 2006; 116:3229-3239. [PubMed: 17060947]

ADIPEX-P® Prescribing Information. 2012. Available from URL:http://www.accessdata.fda.gov/ drugsatfda_docs/label/2012/085128s065lbl.pdf 
Agmo A, Galvan A, Talamantes B. Reward and reinforcement produced by drinking sucrose: two processes that may depend on different neurotransmitters. Pharmacol Biochem Behav. 1995; 52:403-414. [PubMed: 8577808]

Ahituv N, Kavaslar N, Schackwitz W, Ustaszewska A, Collier JM, Hebert S, et al. A PYY Q62P variant linked to human obesity. Hum Mol Genet. 2006; 15:387-391. [PubMed: 16368708]

al'Absi M, Hooker S, Fujiwara K, Kiefer F, von der Goltz C, Cragin T, et al. Circulating leptin levels are associated with increased craving to smoke in abstinent smokers. Pharmacol Biochem Behav. 2011; 97:509-513. [PubMed: 20951159]

Alhadeff AL, Rupprecht LE, Hayes MR. GLP-1 neurons in the nucleus of the solitary tract project directly to the ventral tegmental area and nucleus accumbens to control for food intake. Endocrinology. 2012; 153:647-658. [PubMed: 22128031]

Allison DB, Gadde KM, Garvey WT, Peterson CA, Schwiers ML, Najarian T, et al. Controlled-release phentermine/topiramate in severely obese adults: a randomized controlled trial (EQUIP). Obesity (Silver Spring). 2012; 20:330-342. [PubMed: 22051941]

American Psychiatric Association. Diagnostic and Statistical Manual of Mental Disorders: DSM-5. Fifth Edition. American Psychiatric Publishing; Washington DC: 2013. p. 350

Anderson JW. Orlistat for the management of overweight individuals and obesity: a review of potential for the 60-mg, over-the-counter dosage. Expert Opin Pharmacother. 2007; 8:1733-1742. [PubMed: 17685889]

Angeles-Castellanos M, Mendoza J, Escobar C. Restricted feeding schedules phase shift daily rhythms of c-Fos and protein Per1 immunoreactivity in corticolimbic regions in rats. Neuroscience. 2007; 144:344-355. [PubMed: 17045749]

Apovian CM, Aronne L, Rubino D, Still C, Wyatt H, Burns C, et al. A randomized, phase 3 trial of naltrexone SR/bupropion SR on weight and obesity-related risk factors (COR-II). Obesity (Silver Spring). 2013; 21:935-943. [PubMed: 23408728]

Arita Y, Kihara S, Ouchi N, Takahashi M, Maeda K, Miyagawa J, et al. Paradoxical decrease of an adipose-specific protein, adiponectin, in obesity. Biochem Biophys Res Commun. 1999; 257:79_ 83. [PubMed: 10092513]

Arterburn DE, Bogart A, Sherwood NE, Sidney S, Coleman KJ, Haneuse S, et al. A multisite study of long-term remission and relapse of type 2 diabetes mellitus following gastric bypass. Obes Surg. 2013; 23:93-102. [PubMed: 23161525]

Asnicar MA, Smith DP, Yang DD, Heiman ML, Fox N, Chen YF, et al. Absence of cocaine- and amphetamine-regulated transcript results in obesity in mice fed a high caloric diet. Endocrinology. 2001; 142:4394-4400. [PubMed: 11564703]

Astarita G, Rourke BC, Andersen JB, Fu J, Kim JH, Bennett AF, et al. Postprandial increase of oleoylethanolamide mobilization in small intestine of the Burmese python (Python molurus). Am J Physiol Regul Integr Comp Physiol. 2006; 290:R1407-1412. [PubMed: 16373434]

Astrup A, Madsbad S, Breum L, Jensen TJ, Kroustrup JP, Larsen TM. Effect of tesofensine on bodyweight loss, body composition, and quality of life in obese patients: a randomized, doubleblind, placebo-controlled trial. Lancet. 2008a; 372:1906-1913. [PubMed: 18950853]

Astrup A, Meier DH, Mikkelsen BO, Villumsen JS, Larsen TM. Weight loss produced by tesofensine in patients with Parkinson's or Alzheimer's disease. Obesity (Silver Spring). 2008b; 16:1363-1369. [PubMed: 18356831]

Avenell A, Broom J, Brown TJ, Poobalan A, Aucott L, Stearns SC, et al. Systematic review of the long-term effects and economic consequences of treatments for obesity and implications for health improvement. Health Technol Assess. 2004; 8:iii-iv. 1-182.

Axel AM, Mikkelsen JD, Hansen HH. Tesofensine, a novel triple monoamine reuptake inhibitor, induces appetite suppression by indirect stimulation of alpha1 adrenoceptor and dopamine D1 receptor pathways in the diet-induced obese rat. Neuropsychopharmacology. 2010; 35:1464-1476. [PubMed: 20200509]

Azari EK, Ramachandran D, Weibel S, Arnold M, Romano A, Gaetani S, et al. Vagal afferents are not necessary for the satiety effect of the gut lipid messenger oleoylethanolamide. Am J Physiol Regul Integr Comp Physiol. 2014; 307:R167-178. [PubMed: 24829501] 
Backhed F, Ding H, Wang T, Hooper LV, Koh GY, Nagy A, et al. The gut microbiota as an environmental factor that regulates fat storage. Proc Natl Acad Sci U S A. 2004; 101:1571815723. [PubMed: 15505215]

Baggio LL, Huang Q, Brown TJ, Drucker DJ. Oxyntomodulin and glucagon-like peptide-1 differentially regulate murine food intake and energy expenditure. Gastroenterology. 2004; 127:546-558. [PubMed: 15300587]

Bailey EF. A tasty morsel: the role of the dorsal vagal complex in the regulation of food intake and swallowing. Focus on "BDNF/TrkB signaling interacts with GABAergic system to inhibit rhythmic swallowing in the rat," by Bariohay et al. Am J Physiol Regul Integr Comp Physiol. 2008; 295:R1048-1049. [PubMed: 18753270]

Bala V, Rajagopal S, Kumar DP, Nalli AD, Mahavadi S, Sanyal AJ, et al. Release of GLP-1 and PYY in response to the activation of G protein-coupled bile acid receptor TGR5 is mediated by Epac/ PLC-epsilon pathway and modulated by endogenous H2S. Front Physiol. 2014; 5:420. [PubMed: 25404917]

Ballinger A, McLoughlin L, Medbak S, Clark M. Cholecystokinin is a satiety hormone in humans at physiological post-prandial plasma concentrations. Clin Sci (Lond). 1995; 89:375-381. [PubMed: 7493437]

Batterham RL, Bloom SR. The gut hormone peptide YY regulates appetite. Ann N Y Acad Sci. 2003; 994:162-168. [PubMed: 12851312]

Bays HE, Weinstein R, Law G, Canovatchel W. Canagliflozin: effects in overweight and obese subjects without diabetes mellitus. Obesity (Silver Spring). 2014; 22:1042-1049. [PubMed: 24227660]

Ben-Menachem E, Axelsen M, Johanson EH, Stagge A, Smith U. Predictors of weight loss in adults with topiramate-treated epilepsy. Obes Res. 2003; 11:556-562. [PubMed: 12690085]

Benoit SC, Air EL, Coolen LM, Strauss R, Jackman A, Clegg DJ, et al. The catabolic action of insulin in the brain is mediated by melanocortins. J Neurosci. 2002; 22:9048-9052. [PubMed: 12388611]

Berg AH, Combs TP, Du X, Brownlee M, Scherer PE. The adipocyte-secreted protein Acrp30 enhances hepatic insulin action. Nat Med. 2001; 7:947-953. [PubMed: 11479628]

Berridge KC. Measuring hedonic impact in animals and infants: microstructure of affective taste reactivity patterns. Neurosci Biobehav Rev. 2000; 24:173-198. [PubMed: 10714382]

Berridge KC, Robinson TE. What is the role of dopamine in reward: hedonic impact, reward learning, or incentive salience? Brain Res Brain Res Rev. 1998; 28:309-369. [PubMed: 9858756]

Berryman DE, Glad CA, List EO, Johannsson G. The GH/IGF-1 axis in obesity: pathophysiology and therapeutic considerations. Nat Rev Endocrinol. 2013; 9:346-356. [PubMed: 23568441]

Berthoud HR. Multiple neural systems controlling food intake and body weight. Neurosci Biobehav Rev. 2002; 26:393-428. [PubMed: 12204189]

Berthoud HR. Metabolic and hedonic drives in the neural control of appetite: who is the boss? Curr Opin Neurobiol. 2011; 21:888-896. [PubMed: 21981809]

Bi S, Ladenheim EE, Schwartz GJ, Moran TH. A role for NPY overexpression in the dorsomedial hypothalamus in hyperphagia and obesity of OLETF rats. Am J Physiol Regul Integr Comp Physiol. 2001; 281:R254-260. [PubMed: 11404301]

Bi S, Moran TH. Actions of CCK in the controls of food intake and body weight: lessons from the CCK-A receptor deficient OLETF rat. Neuropeptides. 2002; 36:171-181. [PubMed: 12359507]

Billes SK, Sinnayah P, Cowley MA. Naltrexone/bupropion for obesity: an investigational combination pharmacotherapy for weight loss. Pharmacol Res. 2014; 84:1-11. [PubMed: 24754973]

Bishop FK, Maahs DM, Snell-Bergeon JK, Ogden LG, Kinney GL, Rewers M. Lifestyle risk factors for atherosclerosis in adults with type 1 diabetes. Diab Vasc Dis Res. 2009; 6:269-275. [PubMed: 20368221]

Blache P, Kervran A, Bataille D. Oxyntomodulin and glicentin: brain-gut peptides in the rat. Endocrinology. 1988; 123:2782-2787. [PubMed: 3197645]

Blevins JE, Stanley BG, Reidelberger RD. Brain regions where cholecystokinin suppresses feeding in rats. Brain Res. 2000; 860:1-10. [PubMed: 10727618]

Bloomgarden ZT. Obesity, hypertension, and insulin resistance. Diabetes Care. 2002; 25:2088-2097. [PubMed: 12401761] 
Blundell JE. Is there a role for serotonin (5-hydroxytryptamine) in feeding? Int J Obes. 1977; 1:15-42. [PubMed: 361584]

Blundell J. Pharmacological approaches to appetite suppression. Trends Pharmacol Sci. 1991; 12:147157. [PubMed: 2063481]

Bookout AL, Jeong Y, Downes M, Yu RT, Evans RM, Mangelsdorf DJ. Anatomical profiling of nuclear receptor expression reveals a hierarchical transcriptional network. Cell. 2006; 126:789799. [PubMed: 16923397]

Borgland SL, Chang SJ, Bowers MS, Thompson JL, Vittoz N, Floresco SB, et al. Orexin A/ hypocretin-1 selectively promotes motivation for positive reinforcers. J Neurosci. 2009; 29:1121511225. [PubMed: 19741128]

Bragulat V, Dzemidzic M, Bruno C, Cox CA, Talavage T, Considine RV, et al. Food-related odor probes of brain reward circuits during hunger: a pilot FMRI study. Obesity (Silver Spring). 2010; 18:1566-1571. [PubMed: 20339365]

Brange J, Langkjoer L. Insulin structure and stability. Pharm Biotechnol. 1993; 5:315-350. [PubMed: 8019699]

Bray GA, Hollander P, Klein S, Kushner R, Levy B, Fitchet M, et al. A 6-month randomized, placebocontrolled, dose-ranging trial of topiramate for weight loss in obesity. Obes Res. 2003; 11:722733. [PubMed: 12805393]

Bredella MA, Torriani M, Thomas BJ, Ghomi RH, Brick DJ, Gerweck AV, et al. Peak growth hormone-releasing hormone-arginine-stimulated growth hormone is inversely associated with intramyocellular and intrahepatic lipid content in premenopausal women with obesity. J Clin Endocrinol Metab. 2009; 94:3995-4002. [PubMed: 19602559]

Bremner JD, Krystal JH, Southwick SM, Charney DS. Noradrenergic mechanisms in stress and anxiety: II. Clinical studies. Synapse. 1996; 23:39-51. [PubMed: 8723134]

Broberger C, Johansen J, Johansson C, Schalling M, Hokfelt T. The neuropeptide Y/agouti generelated protein (AGRP) brain circuitry in normal, anorectic, and monosodium glutamate-treated mice. Proc Natl Acad Sci U S A. 1998; 95:15043-15048. [PubMed: 9844012]

Brown TM, Skop BP, Mareth TR. Pathophysiology and management of the serotonin syndrome. Ann Pharmacother. 1996; 30:527-533. [PubMed: 8740336]

Bruijnzeel AW, Corrie LW, Rogers JA, Yamada H. Effects of insulin and leptin in the ventral tegmental area and arcuate hypothalamic nucleus on food intake and brain reward function in female rats. Behav Brain Res. 2011; 219:254-264. [PubMed: 21255613]

Bullock BP, Heller RS, Habener JF. Tissue distribution of messenger ribonucleic acid encoding the rat glucagon-like peptide-1 receptor. Endocrinology. 1996; 137:2968-2978. [PubMed: 8770921]

Callahan HS, Cummings DE, Pepe MS, Breen PA, Matthys CC, Weigle DS. Postprandial suppression of plasma ghrelin level is proportional to ingested caloric load but does not predict intermeal interval in humans. J Clin Endocrinol Metab. 2004; 89:1319-1324. [PubMed: 15001628]

Calu DJ, Chen YW, Kawa AB, Nair SG, Shaham Y. The use of the reinstatement model to study relapse to palatable food seeking during dieting. Neuropharmacology. 2014; 76(Pt B):395-406. [PubMed: 23660229]

Camilleri M. Peripheral mechanisms in appetite regulation. Gastroenterology. 2015; 148(6):12191233. [PubMed: 25241326]

Campfield LA, Smith FJ, Burn P. The OB protein (leptin) pathway--a link between adipose tissue mass and central neural networks. Horm Metab Res. 1996; 28:619-632. [PubMed: 9013731]

Carvelli L, Moron JA, Kahlig KM, Ferrer JV, Sen N, Lechleiter JD, et al. PI 3-kinase regulation of dopamine uptake. J Neurochem. 2002; 81:859-869. [PubMed: 12065645]

Cason AM, Aston-Jones G. Role of orexin/hypocretin in conditioned sucrose-seeking in rats. Psychopharmacology (Berl). 2013; 226:155-165. [PubMed: 23096770]

Cason AM, Smith RJ, Tahsili-Fahadan P, Moorman DE, Sartor GC, Aston-Jones G. Role of orexin/ hypocretin in reward-seeking and addiction: implications for obesity. Physiol Behav. 2010; 100:419-428. [PubMed: 20338186]

Cass WA, Gerhardt GA. Direct in vivo evidence that D2 dopamine receptors can modulate dopamine uptake. Neurosci Lett. 1994; 176:259-263. [PubMed: 7830960]

CDC Report. 2014. http://www.cdc.gov/obesity/data/adult.html 
Chaperon F, Soubrie P, Puech AJ, Thiebot MH. Involvement of central cannabinoid (CB1) receptors in the establishment of place conditioning in rats. Psychopharmacology (Berl). 1998; 135:324-332. [PubMed: 9539255]

Charmot D. Non-systemic drugs: a critical review. Curr Pharm Des. 2012; 18:1434-1445. [PubMed: 22300258]

Chelikani PK, Haver AC, Reidelberger RD. Intravenous infusion of glucagon-like peptide-1 potently inhibits food intake, sham feeding, and gastric emptying in rats. Am J Physiol Regul Integr Comp Physiol. 2005; 288:R1695-1706. [PubMed: 15718384]

Chen AS, Marsh DJ, Trumbauer ME, Frazier EG, Guan XM, Yu H, et al. Inactivation of the mouse melanocortin-3 receptor results in increased fat mass and reduced lean body mass. Nat Genet. 2000; 26:97-102. [PubMed: 10973258]

Chen KY, Muniyappa R, Abel BS, Mullins KP, Staker P, Brychta RJ, et al. RM-493, a melanocortin-4 receptor (MC4R) agonist, increases resting energy expenditure in obese individuals. J Clin Endocrinol Metab. 2015; 100:1639-1645. [PubMed: 25675384]

Chen WJ, Armour S, Way J, Chen G, Watson C, Irving P, et al. Expression cloning and receptor pharmacology of human calcitonin receptors from MCF-7 cells and their relationship to amylin receptors. Mol Pharmacol. 1997; 52:1164-1175. [PubMed: 9396787]

Chen Y, Hu C, Hsu CK, Zhang Q, Bi C, Asnicar M, et al. Targeted disruption of the melaninconcentrating hormone receptor-1 results in hyperphagia and resistance to diet-induced obesity. Endocrinology. 2002; 143:2469-2477. [PubMed: 12072376]

Cho NH, Chan JC, Jang HC, Lim S, Kim HL, Choi SH. Cigarette smoking is an independent risk factor for type 2 diabetes: a four-year community-based prospective study. Clin Endocrinol (Oxf). 2009; 71:679-685. [PubMed: 19508609]

Choi YH, Hartzell D, Azain MJ, Baile CA. TRH decreases food intake and increases water intake and body temperature in rats. Physiol Behav. 2002; 77:1-4. [PubMed: 12213495]

Christensen R, Kristensen PK, Bartels EM, Bliddal H, Astrup A. Efficacy and safety of the weight-loss drug rimonabant: a meta-analysis of randomized trials. Lancet. 2007; 370:1706-1713. [PubMed: 18022033]

Christopoulos G, Perry KJ, Morfis M, Tilakaratne N, Gao Y, Fraser NJ, et al. Multiple amylin receptors arise from receptor activity-modifying protein interaction with the calcitonin receptor gene product. Mol Pharmacol. 1999; 56:235-242. [PubMed: 10385705]

Cincotta AH, Meier AH. Bromocriptine (Ergoset) reduces body weight and improves glucose tolerance in obese subjects. Diabetes Care. 1996; 19:667-670. [PubMed: 8725871]

Clasey JL, Weltman A, Patrie J, Weltman JY, Pezzoli S, Bouchard C, et al. Abdominal visceral fat and fasting insulin are important predictors of 24-hour GH release independent of age, gender, and other physiological factors. J Clin Endocrinol Metab. 2001; 86:3845-3852. [PubMed: 11502822]

Cleary J, Weldon DT, O'Hare E, Billington C, Levine AS. Naloxone effects on sucrose-motivated behavior. Psychopharmacology (Berl). 1996; 126:110-114. [PubMed: 8856829]

Clemmensen C, Finan B, Fischer K, Tom RZ, Legutko B, Sehrer L, et al. Dual melanocortin-4 receptor and GLP-1 receptor agonism amplifies metabolic benefits in diet-induced obese mice. EMBO Mol Med. 2015; 7:288-298. [PubMed: 25652173]

Colantuoni C, Schwenker J, McCarthy J, Rada P, Ladenheim B, Cadet JL, et al. Excessive sugar intake alters binding to dopamine and mu-opioid receptors in the brain. Neuroreport. 2001; 12:35493552. [PubMed: 11733709]

Colditz GA, Willett WC, Stampfer MJ, Manson JE, Hennekens CH, Arky RA, et al. Weight as a risk factor for clinical diabetes in women. Am J Epidemiol. 1990; 132:501-513. [PubMed: 2389754]

Cole S, Mayer HS, Petrovich GD. Orexin/Hypocretin-1 Receptor Antagonism Selectively Reduces Cue-Induced Feeding in Sated Rats and Recruits Medial Prefrontal Cortex and Thalamus. Sci Rep. 2015; 5:16143. [PubMed: 26536818]

Colman E, Golden J, Roberts M, Egan A, Weaver J, Rosebraugh C. The FDA's assessment of two drugs for chronic weight management. N Engl J Med. 2012; 367:1577-1579. [PubMed: 23050510]

Colon-Gonzalez F, Kim GW, Lin JE, Valentino MA, Waldman SA. Obesity pharmacotherapy: what is next? Mol Aspects Med. 2013; 34:71-83. [PubMed: 23103610] 
Cota D, Steiner MA, Marsicano G, Cervino C, Herman JP, Grubler Y, et al. Requirement of cannabinoid receptor type 1 for the basal modulation of hypothalamic-pituitary-adrenal axis function. Endocrinology. 2007; 148:1574-1581. [PubMed: 17194743]

Cottone P, Sabino V, Steardo L, Zorrilla EP. Opioid-dependent anticipatory negative contrast and binge-like eating in rats with limited access to highly preferred food. Neuropsychopharmacology. 2008; 33:524-535. [PubMed: 17443124]

Courtney AE, O'Rourke DM, Maxwell AP. Rapidly progressive renal failure associated with successful pharmacotherapy for obesity. Nephrol Dial Transplant. 2007; 22:621-623. [PubMed: 17121780]

Cowley MA, Pronchuk N, Fan W, Dinulescu DM, Colmers WF, Cone RD. Integration of NPY, AGRP, and melanocortin signals in the hypothalamic paraventricular nucleus: evidence of a cellular basis for the adipostat. Neuron. 1999; 24:155-163. [PubMed: 10677034]

Cowley MA, Smart JL, Rubinstein M, Cerdan MG, Diano S, Horvath TL, et al. Leptin activates anorexigenic POMC neurons through a neural network in the arcuate nucleus. Nature. 2001; 411:480-484. [PubMed: 11373681]

Cristino L, Busetto G, Imperatore R, Ferrandino I, Palomba L, Silvestri C, et al. Obesity-driven synaptic remodeling affects endocannabinoid control of orexinergic neurons. Proc Natl Acad Sci U S A. 2013; 110:E2229-2238. [PubMed: 23630288]

Cryer PE. Minireview: Glucagon in the pathogenesis of hypoglycemia and hyperglycemia in diabetes. Endocrinology. 2012; 153:1039-1048. [PubMed: 22166985]

Cuchel M, Rader DJ. Microsomal transfer protein inhibition in humans. Curr Opin Lipidol. 2013; 24:246-250. [PubMed: 23594709]

Cummings DE. Ghrelin and the short- and long-term regulation of appetite and body weight. Physiol Behav. 2006; 89:71-84. [PubMed: 16859720]

Cummings DE, Purnell JQ, Frayo RS, Schmidova K, Wisse BE, Weigle DS. A preprandial rise in plasma ghrelin levels suggests a role in meal initiation in humans. Diabetes. 2001; 50:1714-1719. [PubMed: 11473029]

Davidson IW, Salter JM, Best CH. Calorigenic action of glucagon. Nature. 1957; 180:1124.

Davies BT, Wellman PJ. Effects on ingestive behavior in rats of the alpha 1-adrenoceptor agonist cirazoline. Eur J Pharmacol. 1992; 210:11-16. [PubMed: 1350985]

Davis JF, Choi DL, Schurdak JD, Fitzgerald MF, Clegg DJ, Lipton JW, et al. Leptin regulates energy balance and motivation through action at distinct neural circuits. Biol Psychiatry. 2011; 69:668674. [PubMed: 21035790]

Davis LM, Michaelides M, Cheskin LJ, Moran TH, Aja S, Watkins PA, et al. Bromocriptine administration reduces hyperphagia and adiposity and differentially affects dopamine D2 receptor and transporter binding in leptin-receptor-deficient Zucker rats and rats with diet-induced obesity. Neuroendocrinology. 2009; 89:152-162. [PubMed: 18984941]

Davis JF, Tracy AL, Schurdak JD, Tschop MH, Lipton JW, Clegg DJ, et al. Exposure to elevated levels of dietary fat attenuates psychostimulant reward and mesolimbic dopamine turnover in the rat. Behav Neurosci. 2008; 122:1257-1263. [PubMed: 19045945]

de Lecea L, Kilduff TS, Peyron C, Gao X, Foye PE, Danielson PE, et al. The hypocretins: hypothalamus-specific peptides with neuroexcitatory activity. Proc Natl Acad Sci U S A. 1998; 95:322-327. [PubMed: 9419374]

De Olmos, JS., Heimer, L. The concepts of the ventral striatopallidal system and extended amygdala. In: McGinty, JF., editor. Advancing from the ventral striatum to the extended amygdala: Implications for neuropsychiatry and drug abuse. The New York Academy of Sciences; New York: 1999. p. 1-32.

De Simone G, Di Fiore A, Supuran CT. Are carbonic anhydrase inhibitors suitable for obtaining antiobesity drugs? Curr. Pharm. Des. 2008; 14:655-660. [PubMed: 18336311]

Degen L, Matzinger D, Drewe J, Beglinger C. The effect of cholecystokinin in controlling appetite and food intake in humans. Peptides. 2001; 22:1265-1269. [PubMed: 11457519]

Di Marzo V, Ligresti A, Cristino L. The endocannabinoid system as a link between homoeostatic and hedonic pathways involved in energy balance regulation. Int J Obes (Lond). 2009; 33(Suppl 2):S18-24. [PubMed: 19528974] 
Dickson SL, Hrabovszky E, Hansson C, Jerlhag E, Alvarez-Crespo M, Skibicka KP, et al. Blockade of central nicotine acetylcholine receptor signaling attenuate ghrelin-induced food intake in rodents. Neuroscience. 2010; 171:1180-1186. [PubMed: 20933579]

Dickson SL, Shirazi RH, Hansson C, Bergquist F, Nissbrandt H, Skibicka KP. The glucagon-like peptide 1 (GLP-1) analogue, exendin-4, decreases the rewarding value of food: a new role for mesolimbic GLP-1 receptors. J Neurosci. 2012; 32:4812-4820. [PubMed: 22492036]

DiPatrizio NV, Astarita G, Schwartz G, Li X, Piomelli D. Endocannabinoid signal in the gut controls dietary fat intake. Proc Natl Acad Sci U S A. 2011; 108:12904-12908. [PubMed: 21730161]

DiPatrizio NV, Igarashi M, Narayanaswami V, Murray C, Gancayco J, Russell A, et al. Fasting stimulates 2-AG biosynthesis in the small intestine: role of cholinergic pathways. Am J Physiol Regul Integr Comp Physiol. 2015; 309:R805-813. [PubMed: 26290104]

DiPatrizio NV, Joslin A, Jung KM, Piomelli D. Endocannabinoid signaling in the gut mediates preference for dietary unsaturated fats. FASEB J. 2013; 27:2513-2520. [PubMed: 23463697]

DiPatrizio NV, Piomelli D. Intestinal lipid-derived signals that sense dietary fat. J Clin Invest. 2015; 125:891-898. [PubMed: 25642767]

Druce MR, Wren AM, Park AJ, Milton JE, Patterson M, Frost G, et al. Ghrelin increases food intake in obese as well as lean subjects. Int J Obes (Lond). 2005; 29:1130-1136. [PubMed: 15917842]

Drucker DJ. Enhancing the action of incretin hormones: a new whey forward? Endocrinology. 2006; 147:3171-3172. [PubMed: 16777979]

Drucker DJ. The role of gut hormones in glucose homeostasis. J Clin Invest. 2007; 117:24-32. [PubMed: 17200703]

Drucker DJ, Asa S. Glucagon gene expression in vertebrate brain. J Biol Chem. 1988; 263:1347513478. [PubMed: 2901414]

Dufresne M, Seva C, Fourmy D. Cholecystokinin and gastrin receptors. Physiol Rev. 2006; 86:805847. [PubMed: 16816139]

Dumont Y, Fournier A, St-Pierre S, Quirion R. Characterization of neuropeptide Y binding sites in rat brain membrane preparations using [125I][Leu31,Pro34]peptide YY and [125I]peptide YY3-36 as selective Y1 and Y2 radioligands. J Pharmacol Exp Ther. 1995; 272:673-680. [PubMed: 7853181]

Dushay J, Gao C, Gopalakrishnan GS, Crawley M, Mitten EK, Wilker E, et al. Short-term exenatide treatment leads to significant weight loss in a subset of obese women without diabetes. Diabetes Care. 2012; 35:4-11. [PubMed: 22040840]

Dwoskin LP, Rauhut AS, King-Pospisil KA, Bardo MT. Review of the pharmacology and clinical profile of bupropion, an antidepressant and tobacco use cessation agent. CNS Drug Rev. 2006; 12:178-207. [PubMed: 17227286]

Ebihara K, Ogawa Y, Katsuura G, Numata Y, Masuzaki H, Satoh N, et al. Involvement of agoutirelated protein, an endogenous antagonist of hypothalamic melanocortin receptor, in leptin action. Diabetes. 1999; 48:2028-2033. [PubMed: 10512369]

Eckel RH, Grundy SM, Zimmet PZ. The metabolic syndrome. Lancet. 2005; 365:1415-1428. [PubMed: 15836891]

Eggink HM, Soeters MR, Pols TWH. TGR5 ligands as potential therapeutics in inflammatory diseases. Int. J. Interferon Cytokine Mediator Res. 2014; 6:27-38.

Eipper BA, Mains RE. Structure and biosynthesis of proadrenocorticotropin/endorphin and related peptides. Endocr Rev. 1980; 1:1-27. [PubMed: 6262069]

Elias CF, Lee C, Kelly J, Aschkenasi C, Ahima RS, Couceyro PR, et al. Leptin activates hypothalamic CART neurons projecting to the spinal cord. Neuron. 1998; 21:1375-1385. [PubMed: 9883730]

Elkind-Hirsch K, Marrioneaux O, Bhushan M, Vernor D, Bhushan R. Comparison of single and combined treatment with exenatide and metformin on menstrual cyclicity in overweight women with polycystic ovary syndrome. J Clin Endocrinol Metab. 2008; 93:2670-2678. [PubMed: 18460557]

Elmquist JK, Maratos-Flier E, Saper CB, Flier JS. Unraveling the central nervous system pathways underlying responses to leptin. Nat Neurosci. 1998; 1:445-450. [PubMed: 10196541] 
European Medicines Agency. The European Medicines Agency recommends suspension of the marketing authorization of Acomplia. 2008. Available from: http://www.ema.europa.eu/docs/ en_GB/document_library/Press_release/2009/11/WC500014774.pdf

Evans RM, Barish GD, Wang YX. PPARs and the complex journey to obesity. Nat Med. 2004; 10:355-361. [PubMed: 15057233]

Everitt BJ, Fray P, Kostarczyk E, Taylor S, Stacey P. Studies of instrumental behavior with sexual reinforcement in male rats (Rattus norvegicus): I. Control by brief visual stimuli paired with a receptive female. J Comp Psychol. 1987; 101:395-406. [PubMed: 3691062]

Everitt BJ, Robbins TW. Neural systems of reinforcement for drug addiction: from actions to habits to compulsion. Nat Neurosci. 2005; 8:1481-1489. [PubMed: 16251991]

Fang S, Suh JM, Reilly SM, Yu E, Osborn O, Lackey D, et al. Intestinal FXR agonism promotes adipose tissue browning and reduces obesity and insulin resistance. Nat Med. 2015; 21:159-165. [PubMed: 25559344]

FDA. FDA Approves Orlistat for Over-the-Counter Use. 2007. http://www.fda.gov/NewsEvents/ Newsroom/PressAnnouncements/2007/ucm108839.htm

FDA draft guidance. Guidance for Industry Developing Products for Weight Management. 2007. www.fda.gov/downloads/Drugs/.../Guidances/ucm071612.pdf

FDA draft guidance. Department of Health and Human Services. [Accessed 25 Aug 2011] Guidance for industry: Patient-reported outcome measures: Use in medical product development to support labeling claims: draft guidance. 2009. http://www.fda.gov/downloads/Drugs/ GuidanceComplianceRegulatoryInformation/Guidances/UCM193282.pdf

FDA. FDA Approves New Treatment for Type 2 Diabetes. 2010. http://www.fda.gov/NewsEvents/ Newsroom/PressAnnouncements/ucm198638.htm

FDA drug safety communication. Completed safety review of Xenical/Alli (orlistat) and severe liver injury. 2010. http://www.fda.gov/Drugs/DrugSafety/ PostmarketDrugSafetyInformationforPatientsandProviders/ucm213038.htm

FDA. FDA approves belviq to treat some overweight or obese adults. 2012a. http://www.fda.gov/ NewsEvents/Newsroom/PressAnnouncements/ucm309993.htm

FDA. FDA approves weight-management drug Qsymia. 2012b. http://www.fda.gov/NewsEvents/ Newsroom/PressAnnouncements/ucm312468.htm

FDA. FDA approves weight-management drug Contrave. 2014a. http://www.fda.gov/NewsEvents/ Newsroom/PressAnnouncements/ucm413896.htm

FDA. FDA approves weight-management drug Saxenda. 2014b. http://www.fda.gov/NewsEvents/ Newsroom/PressAnnouncements/ucm427913.htm

Fei H, Okano HJ, Li C, Lee GH, Zhao C, Darnell R, et al. Anatomic localization of alternatively spliced leptin receptors (Ob-R) in mouse brain and other tissues. Proc Natl Acad Sci U S A. 1997; 94:7001-7005. [PubMed: 9192681]

Fekete C, Legradi G, Mihaly E, Huang QH, Tatro JB, Rand WM, et al. alpha-Melanocyte-stimulating hormone is contained in nerve terminals innervating thyrotropin-releasing hormone-synthesizing neurons in the hypothalamic paraventricular nucleus and prevents fasting-induced suppression of prothyrotropin-releasing hormone gene expression. J Neurosci. 2000; 20:1550-1558. [PubMed: 10662844]

Ferraz RR, Tiselius HG, Heilberg IP. Fat malabsorption induced by gastrointestinal lipase inhibitor leads to an increase in urinary oxalate excretion. Kidney Int. 2004; 66:676-682. [PubMed: 15253722]

Figlewicz DP, Bennett J, Evans SB, Kaiyala K, Sipols AJ, Benoit SC. Intraventricular insulin and leptin reverse place preference conditioned with high-fat diet in rats. Behav Neurosci. 2004; 118:479-487. [PubMed: 15174925]

Figlewicz DP, Bennett JL, Naleid AM, Davis C, Grimm JW. Intraventricular insulin and leptin decrease sucrose self-administration in rats. Physiol Behav. 2006; 89:611-616. [PubMed: 17045623]

Figlewicz DP, Evans SB, Murphy J, Hoen M, Baskin DG. Expression of receptors for insulin and leptin in the ventral tegmental area/substantia nigra (VTA/SN) of the rat. Brain Res. 2003; 964:107-115. [PubMed: 12573518] 
Figlewicz DP, Higgins MS, Ng-Evans SB, Havel PJ. Leptin reverses sucrose-conditioned place preference in food-restricted rats. Physiol Behav. 2001; 73:229-234. [PubMed: 11399316]

Figlewicz DP, Szot P, Chavez M, Woods SC, Veith RC. Intraventricular insulin increases dopamine transporter mRNA in rat VTA/substantia nigra. Brain Res. 1994; 644:331-334. [PubMed: 8050044]

Fleming JW, McClendon KS, Riche DM. New obesity agents: lorcaserin and phentermine/topiramate. Ann Pharmacother. 2013; 47:1007-1016. [PubMed: 23800750]

Flint A, Raben A, Ersboll AK, Holst JJ, Astrup A. The effect of physiological levels of glucagon-like peptide- 1 on appetite, gastric emptying, energy and substrate metabolism in obesity. Int J Obes Relat Metab Disord. 2001; 25:781-792. [PubMed: 11439290]

Foote SL, Bloom FE, Aston-Jones G. Nucleus locus ceruleus: new evidence of anatomical and physiological specificity. Physiol Rev. 1983; 63:844-914. [PubMed: 6308694]

Forman BM, Goode E, Chen J, Oro AE, Bradley DJ, Perlmann T, et al. Identification of a nuclear receptor that is activated by farnesol metabolites. Cell. 1995; 81:687-693. [PubMed: 7774010]

Frassetto A, Zhang J, Lao JZ, White A, Metzger JM, Fong TM, et al. Reduced sensitivity to dietinduced obesity in mice carrying a mutant 5-HT6 receptor. Brain Res. 2008; 1236:140-144. [PubMed: 18755168]

Freund TF, Katona I, Piomelli D. Role of endogenous cannabinoids in synaptic signaling. Physiol Rev. 2003; 83:1017-1066. [PubMed: 12843414]

Fride E. Endocannabinoids in the central nervous system--an overview. Prostaglandins Leukot Essent Fatty Acids. 2002; 66:221-233. [PubMed: 12052038]

Friedman A, Lax E, Dikshtein Y, Abraham L, Flaumenhaft Y, Sudai E, et al. Electrical stimulation of the lateral habenula produces an inhibitory effect on sucrose self-administration. Neuropharmacology. 2011; 60:381-387. [PubMed: 20955718]

Fruebis J, Tsao TS, Javorschi S, Ebbets-Reed D, Erickson MR, Yen FT, et al. Proteolytic cleavage product of 30-kDa adipocyte complement-related protein increases fatty acid oxidation in muscle and causes weight loss in mice. Proc Natl Acad Sci U S A. 2001; 98:2005-2010. [PubMed: 11172066]

Fu J, Astarita G, Gaetani S, Kim J, Cravatt BF, Mackie K, et al. Food intake regulates oleoylethanolamide formation and degradation in the proximal small intestine. J Biol Chem. 2007; 282:1518-1528. [PubMed: 17121838]

Fu J, Gaetani S, Oveisi F, Lo Verme J, Serrano A, Rodriguez De Fonseca F, et al. Oleylethanolamide regulates feeding and body weight through activation of the nuclear receptor PPAR-alpha. Nature. 2003; 425:90-93. [PubMed: 12955147]

Fu J, Oveisi F, Gaetani S, Lin E, Piomelli D. Oleoylethanolamide, an endogenous PPAR-alpha agonist, lowers body weight and hyperlipidemia in obese rats. Neuropharmacology. 2005; 48:1147-1153. [PubMed: 15910890]

Fujimori Y, Katsuno K, Nakashima I, Ishikawa-Takemura Y, Fujikura H, Isaji M. Remogliflozin etabonate, in a novel category of selective low-affinity sodium glucose cotransporter (SGLT2) inhibitors, exhibits antidiabetic efficacy in rodent models. J Pharmacol Exp Ther. 2008; 327:268276. [PubMed: 18583547]

Fuke S, Suo S, Takahashi N, Koike H, Sasagawa N, Ishiura S. The VNTR polymorphism of the human dopamine transporter (DAT1) gene affects gene expression. Pharmacogenomics J. 2001; 1:152156. [PubMed: 11911442]

Fulton S, Pissios P, Manchon RP, Stiles L, Frank L, Pothos EN, et al. Leptin regulation of the mesoaccumbens dopamine pathway. Neuron. 2006; 51:811-822. [PubMed: 16982425]

Gadde KM, Allison DB. Combination pharmaceutical therapies for obesity. Expert Opin Pharmacother. 2009; 10:921-925. [PubMed: 19351275]

Gadde KM, Allison DB, Ryan DH, Peterson CA, Troupin B, Schwiers ML, et al. Effects of low-dose, controlled-release, phentermine plus topiramate combination on weight and associated comorbidities in overweight and obese adults (CONQUER): a randomized, placebo-controlled, phase 3 trial. Lancet. 2011; 377:1341-1352. [PubMed: 21481449]

Gadde KM, Franciscy DM, Wagner HR 2nd, Krishnan KR. Zonisamide for weight loss in obese adults: a randomized controlled trial. JAMA. 2003; 289:1820-1825. [PubMed: 12684361] 
Gaetani S, Fu J, Cassano T, Dipasquale P, Romano A, Righetti L, et al. The fat-induced satiety factor oleoylethanolamide suppresses feeding through central release of oxytocin. J Neurosci. 2010; 30:8096-8101. [PubMed: 20554860]

Gaetani S, Oveisi F, Piomelli D. Modulation of meal pattern in the rat by the anorexic lipid mediator oleoylethanolamide. Neuropsychopharmacology. 2003; 28:1311-1316. [PubMed: 12700681]

Gannon KS, Heal DJ, Cheetham SC, Jackson HC, Seeley HC, Melendez RJ, et al. PRX-07034, a potent and selective 5-HT6 receptor antagonist, reduces food intake and body weight in rats. J. Pharmacol. Sci. 2006; 101:124.

Garcia BG, Wei Y, Moron JA, Lin RZ, Javitch JA, Galli A. Akt is essential for insulin modulation of amphetamine-induced human dopamine transporter cell-surface redistribution. Mol Pharmacol. 2005; 68:102-109. [PubMed: 15795321]

Garfield AS, Heisler LK. Pharmacological targeting of the serotonergic system for the treatment of obesity. J Physiol. 2009; 587:49-60. [PubMed: 19029184]

Garvey WT, Ryan DH, Look M, Gadde KM, Allison DB, Peterson CA, et al. Two-year sustained weight loss and metabolic benefits with controlled-release phentermine/topiramate in obese and overweight adults (SEQUEL): a randomized, placebo-controlled, phase 3 extension study. Am J Clin Nutr. 2012; 95:297-308. [PubMed: 22158731]

Gearhardt AN, Corbin WR, Brownell KD. Preliminary validation of the Yale Food Addiction Scale. Appetite. 2009; 52(2):430-436. [PubMed: 19121351]

Gearhardt AN, Roberto CA, Seamans MJ, Corbin WR, Brownell KD. Preliminary validation of the Yale Food Addiction Scale for children. Eat Behav. 2013; 14(4):508-512. [PubMed: 24183146]

Gearhardt AN, White MA, Masheb RM, Morgan PT, Crosby RD, Grilo CM. An examination of the food addiction construct in obese patients with binge eating disorder. Int J Eat Disord. 2012; 45(5):657-663. [PubMed: 22684991]

Geiger BM, Behr GG, Frank LE, Caldera-Siu AD, Beinfeld MC, Kokkotou EG, et al. Evidence for defective mesolimbic dopamine exocytosis in obesity-prone rats. FASEB J. 2008; 22:2740-2746. [PubMed: 18477764]

George M, Rajaram M, Shanmugam E. New and emerging drug molecules against obesity. J Cardiovasc Pharmacol Ther. 2014; 19:65-76. [PubMed: 24064009]

Georgescu D, Sears RM, Hommel JD, Barrot M, Bolanos CA, Marsh DJ, et al. The hypothalamic neuropeptide melanin-concentrating hormone acts in the nucleus accumbens to modulate feeding behavior and forced-swim performance. J Neurosci. 2005; 25:2933-2940. [PubMed: 15772353]

Ghitza UE, Gray SM, Epstein DH, Rice KC, Shaham Y. The anxiogenic drug yohimbine reinstates palatable food seeking in a rat relapse model: a role of CRF1 receptors. Neuropsychopharmacology. 2006; 31:2188-2196. [PubMed: 16341025]

Giuliano C, Robbins TW, Nathan PJ, Bullmore ET, Everitt BJ. Inhibition of opioid transmission at the mu-opioid receptor prevents both food seeking and binge-like eating. Neuropsychopharmacology. 2012; 37:2643-2652. [PubMed: 22805601]

Goldman CK, Marino L, Leibowitz SF. Postsynaptic alpha 2-noradrenergic receptors mediate feeding induced by paraventricular nucleus injection of norepinephrine and clonidine. Eur J Pharmacol. 1985; 115:11-19. [PubMed: 2995083]

Gomez R, Navarro M, Ferrer B, Trigo JM, Bilbao A, Del Arco I, et al. A peripheral mechanism for CB1 cannabinoid receptor-dependent modulation of feeding. J Neurosci. 2002; 22:9612-9617. [PubMed: 12417686]

Gong JP, Onaivi ES, Ishiguro H, Liu QR, Tagliaferro PA, Brusco A, et al. Cannabinoid CB2 receptors: immunohistochemical localization in rat brain. Brain Res. 2006; 1071:10-23. [PubMed: 16472786]

Gordon DA, Jamil H. Progress towards understanding the role of microsomal triglyceride transfer protein in apolipoprotein-B lipoprotein assembly. Biochim Biophys Acta. 2000; 1486:72-83. [PubMed: 10856714]

Grandt D, Schimiczek M, Beglinger C, Layer P, Goebell H, Eysselein VE, et al. Two molecular forms of peptide YY (PYY) are abundant in human blood: characterization of a radioimmunoassay recognizing PYY 1-36 and PYY 3-36. Regul Pept. 1994a; 51:151-159. [PubMed: 8059011] 
Grandt D, Schimiczek M, Struk K, Shively J, Eysselein VE, Goebell H, et al. Characterization of two forms of peptide YY, PYY(1-36) and PYY(3-36), in the rabbit. Peptides. 1994b; 15:815-820. [PubMed: 7984499]

Greenberg D, Smith GP. The controls of fat intake. Psychosom Med. 1996; 58:559-569. [PubMed: 8948004]

Greenway FL, Whitehouse MJ, Guttadauria M, Anderson JW, Atkinson RL, Fujioka K, et al. Rational design of a combination medication for the treatment of obesity. Obesity (Silver Spring). 2009; 17:30-39. [PubMed: 18997675]

Grill HJ, Hayes MR. Hindbrain neurons as an essential hub in the neuroanatomically distributed control of energy balance. Cell Metab. 2012; 16:296-309. [PubMed: 22902836]

Gros L, Thorens B, Bataille D, Kervran A. Glucagon-like peptide-1-(7-36) amide, oxyntomodulin, and glucagon interact with a common receptor in a somatostatin-secreting cell line. Endocrinology. 1993; 133:631-638. [PubMed: 8102095]

Gruetzmann R, Beuck M, Mueller U, Nielsch U. Bay 13-9952 (implitapide), an inhibitor of microsomal triglyceride transfer protein (MTP), blocks secretion of Apo-B lipoproteins. Atherosclerosis. 2000; 151:91-92.

Grundy SM. A constellation of complications: the metabolic syndrome. Clin Cornerstone. 2005; 7:3645. [PubMed: 16473259]

Gu G, Roland B, Tomaselli K, Dolman CS, Lowe C, Heilig JS. Glucagon-like peptide-1 in the rat brain: distribution of expression and functional implication. J Comp Neurol. 2013; 521:22352261. [PubMed: 23238833]

Gustafson A, King C, Rey JA. Lorcaserin (Belviq): A Selective Serotonin 5-HT2C Agonist In the Treatment of Obesity. P T. 2013; 38:525-534. [PubMed: 24273398]

Gutzwiller JP, Degen L, Heuss L, Beglinger C. Glucagon-like peptide 1 (GLP-1) and eating. Physiol Behav. 2004; 82:17-19. [PubMed: 15234584]

Haass-Koffler CL, Aoun EG, Swift RM, de la Monte SM, Kenna GA, Leggio L. Leptin levels are reduced by intravenous ghrelin administration and correlated with cue-induced alcohol craving. Transl Psychiatry. 2015; 5:e646. [PubMed: 26418274]

Hahn TM, Breininger JF, Baskin DG, Schwartz MW. Coexpression of Agrp and NPY in fastingactivated hypothalamic neurons. Nat Neurosci. 1998; 1:271-272. [PubMed: 10195157]

Halaas JL, Gajiwala KS, Maffei M, Cohen SL, Chait BT, Rabinowitz D, et al. Weight-reducing effects of the plasma protein encoded by the obese gene. Science. 1995; 269:543-546. [PubMed: 7624777]

Hampp C, Kang EM, Borders-Hemphill V. Use of prescription antiobesity drugs in the United States. Pharmacotherapy. 2013; 33:1299-1307. [PubMed: 24019195]

Hara J, Beuckmann CT, Nambu T, Willie JT, Chemelli RM, Sinton CM, et al. Genetic ablation of orexin neurons in mice results in narcolepsy, hypophagia, and obesity. Neuron. 2001; 30:345354. [PubMed: 11394998]

Harrold JA, Dovey T, Cai XJ, Halford JC, Pinkney J. Autoradiographic analysis of ghrelin receptors in the rat hypothalamus. Brain Res. 2008; 1196:59-64. [PubMed: 18234162]

Harrold JA, Dovey TM, Blundell JE, Halford JC. CNS regulation of appetite. Neuropharmacology. 2012; 63:3-17. [PubMed: 22313528]

Harrold JA, Elliott JC, King PJ, Widdowson PS, Williams G. Down-regulation of cannabinoid-1 (CB-1) receptors in specific extrahypothalamic regions of rats with dietary obesity: a role for endogenous cannabinoids in driving appetite for palatable food? Brain Res. 2002; 952:232-238. [PubMed: 12376184]

Hatoum IJ, Stylopoulos N, Vanhoose AM, Boyd KL, Yin DP, Ellacott KL, et al. Melanocortin-4 receptor signaling is required for weight loss after gastric bypass surgery. J Clin Endocrinol Metab. 2012; 97:E1023-1031. [PubMed: 22492873]

Hauptman J, Lucas C, Boldrin MN, Collins H, Segal KR. Orlistat in the long-term treatment of obesity in primary care settings. Arch Fam Med. 2000; 9:160-167. [PubMed: 10693734]

Haynes AC, Chapman H, Taylor C, Moore GB, Cawthorne MA, Tadayyon M, et al. Anorectic, thermogenic and anti-obesity activity of a selective orexin-1 receptor antagonist in ob/ob mice. Regul Pept. 2002; 104:153-159. [PubMed: 11830290] 
Heal DJ, Gosden J, Smith SL. Regulatory challenges for new drugs to treat obesity and comorbid metabolic disorders. Br J Clin Pharmacol. 2009; 68:861-874. [PubMed: 20002080]

Heal DJ, Gosden J, Smith SL. What is the prognosis for new centrally-acting anti-obesity drugs? Neuropharmacology. 2012; 63:132-146. [PubMed: 22313529]

Heinz A, Goldman D, Jones DW, Palmour R, Hommer D, Gorey JG, et al. Genotype influences in vivo dopamine transporter availability in human striatum. Neuropsychopharmacology. 2000; 22:133139. [PubMed: 10649826]

Hendricks EJ, Srisurapanont M, Schmidt SL, Haggard M, Souter S, Mitchell CL, et al. Addiction potential of phentermine prescribed during long-term treatment of obesity. Int J Obes (Lond). 2014; 38:292-298. [PubMed: 23736363]

Hervieu GJ, Cluderay JE, Harrison D, Meakin J, Maycox P, Nasir S, et al. The distribution of the mRNA and protein products of the melanin-concentrating hormone $(\mathrm{MCH})$ receptor gene, slc-1, in the central nervous system of the rat. Eur J Neurosci. 2000; 12:1194-1216. [PubMed: 10762350]

Hervieu GJ, Cluderay JE, Harrison DC, Roberts JC, Leslie RA. Gene expression and protein distribution of the orexin-1 receptor in the rat brain and spinal cord. Neuroscience. 2001; 103:777-797. [PubMed: 11274794]

Heymsfield SB, Greenberg AS, Fujioka K, Dixon RM, Kushner R, Hunt T, et al. Recombinant leptin for weight loss in obese and lean adults: a randomized, controlled, dose-escalation trial. JAMA. 1999; 282:1568-1575. [PubMed: 10546697]

Heymsfield SB, Segal KR, Hauptman J, Lucas CP, Boldrin MN, Rissanen A, et al. Effects of weight loss with orlistat on glucose tolerance and progression to type 2 diabetes in obese adults. Arch Intern Med. 2000; 160:1321-1326. [PubMed: 10809036]

Hillebrand JJ, de Wied D, Adan RA. Neuropeptides, food intake and body weight regulation: a hypothalamic focus. Peptides. 2002; 23:2283-2306. [PubMed: 12535710]

Hodge RJ, Nunez DJ. Therapeutic potential of Takeda-G-protein-receptor-5 (TGR5) agonists. Hope or hype? Diabetes Obes Metab. 2016; 18:439-443. [PubMed: 26818602]

Hogan S, Fleury A, Hadvary P, Lengsfeld H, Meier MK, Triscari J, et al. Studies on the antiobesity activity of tetrahydrolipstatin, a potent and selective inhibitor of pancreatic lipase. Int J Obes. 1987; 11(Suppl 3):35-42. [PubMed: 3440690]

Hollander PA, Levy P, Fineman MS, Maggs DG, Shen LZ, Strobel SA, et al. Pramlintide as an adjunct to insulin therapy improves long-term glycemic and weight control in patients with type 2 diabetes: a 1-year randomized controlled trial. Diabetes Care. 2003; 26:784-790. [PubMed: 12610038]

Hommel JD, Trinko R, Sears RM, Georgescu D, Liu ZW, Gao XB, et al. Leptin receptor signaling in midbrain dopamine neurons regulates feeding. Neuron. 2006; 51:801-810. [PubMed: 16982424]

Hotta K, Funahashi T, Arita Y, Takahashi M, Matsuda M, Okamoto Y, et al. Plasma concentrations of a novel, adipose-specific protein, adiponectin, in type 2 diabetic patients. Arterioscler Thromb Vasc Biol. 2000; 20:1595-1599. [PubMed: 10845877]

Howell LL, Cunningham KA. Serotonin 5-HT2 receptor interactions with dopamine function: implications for therapeutics in cocaine use disorder. Pharmacol Rev. 2015; 67:176-197. [PubMed: 25505168]

Hughes TE, Kim DD, Marjason J, Proietto J, Whitehead JP, Vath JE. Ascending dose-controlled trial of beloranib, a novel obesity treatment for safety, tolerability, and weight loss in obese women. Obesity (Silver Spring). 2013; 21:1782-1788. [PubMed: 23512440]

Hussain MM, Bakillah A. New approaches to target microsomal triglyceride transfer protein. Curr Opin Lipidol. 2008; 19:572-578. [PubMed: 18957879]

Huszar D, Lynch CA, Fairchild-Huntress V, Dunmore JH, Fang Q, Berkemeier LR, et al. Targeted disruption of the melanocortin-4 receptor results in obesity in mice. Cell. 1997; 88:131-141. [PubMed: 9019399]

Hyde TM, Miselis RR. Effects of area postrema/caudal medial nucleus of solitary tract lesions on food intake and body weight. Am J Physiol. 1983; 244:R577-587. [PubMed: 6837771] 
Ibrahim N, Bosch MA, Smart JL, Qiu J, Rubinstein M, Ronnekleiv OK, et al. Hypothalamic proopiomelanocortin neurons are glucose responsive and express K(ATP) channels. Endocrinology. 2003; 144:1331-1340. [PubMed: 12639916]

Igarashi M, DiPatrizio NV, Narayanaswami V, Piomelli D. Feeding-induced oleoylethanolamide mobilization is disrupted in the gut of diet-induced obese rodents. Biochim Biophys Acta. 2015; 1851:1218-1226. [PubMed: 26024927]

Ignar DM, Goetz AS, Noble KN, Carballo LH, Stroup AE, Fisher JC, et al. Regulation of ingestive behaviors in the rat by GSK1521498, a novel micro-opioid receptor-selective inverse agonist. J Pharmacol Exp Ther. 2011; 339:24-34. [PubMed: 21712426]

Ioannides-Demos LL, Piccenna L, McNeil JJ. Pharmacotherapies for obesity: past, current, and future therapies. J Obes. 2011; 2011:179674. [PubMed: 21197148]

Izzo AA, Piscitelli F, Capasso R, Aviello G, Romano B, Borrelli F, et al. Peripheral endocannabinoid dysregulation in obesity: relation to intestinal motility and energy processing induced by food deprivation and re-feeding. Br J Pharmacol. 2009; 158:451-461. [PubMed: 19371345]

Jaber M, Jones S, Giros B, Caron MG. The dopamine transporter: a crucial component regulating dopamine transmission. Mov Disord. 1997; 12:629-633. [PubMed: 9380041]

Jackson VM, Price DA, Carpino PA. Investigational drugs in Phase II clinical trials for the treatment of obesity: implications for future development of novel therapies. Expert Opin Investig Drugs. 2014; 23:1055-1066.

Jamshidi N, Taylor DA. Anandamide administration into the ventromedial hypothalamus stimulates appetite in rats. Br J Pharmacol. 2001; 134:1151-1154. [PubMed: 11704633]

Jerlhag E, Egecioglu E, Dickson SL, Douhan A, Svensson L, Engel JA. Ghrelin administration into tegmental areas stimulates locomotor activity and increases extracellular concentration of dopamine in the nucleus accumbens. Addict Biol. 2007; 12:6-16. [PubMed: 17407492]

Jerlhag E, Egecioglu E, Landgren S, Salome N, Heilig M, Moechars D, et al. Requirement of central ghrelin signaling for alcohol reward. Proc Natl Acad Sci U S A. 2009; 106:11318-11323. [PubMed: 19564604]

Jessen N, Djurhuus CB, Jorgensen JO, Jensen LS, Moller N, Lund S, et al. Evidence against a role for insulin-signaling proteins PI 3-kinase and Akt in insulin resistance in human skeletal muscle induced by short-term GH infusion. Am J Physiol Endocrinol Metab. 2005; 288:E194-199. [PubMed: 15339744]

Jiang C, Xie C, Lv Y, Li J, Krausz KW, Shi J, et al. Intestine-selective farnesoid X receptor inhibition improves obesity-related metabolic dysfunction. Nat Commun. 2015; 6:10166. [PubMed: 26670557]

Johannsson G. Management of adult growth hormone deficiency. Endocrinol Metab Clin North Am. 2007; 36:203-220. [PubMed: 17336741]

Johnson PM, Kenny PJ. Dopamine D2 receptors in addiction-like reward dysfunction and compulsive eating in obese rats. Nat Neurosci. 2010; 13:635-641. [PubMed: 20348917]

Jorgensen JO, Pedersen SB, Borglum J, Moller N, Schmitz O, Christiansen JS, et al. Fuel metabolism, energy expenditure, and thyroid function in growth hormone-treated obese women: a doubleblind placebo-controlled study. Metabolism. 1994; 43:872-877. [PubMed: 8028511]

Judge MK, Zhang J, Tumer N, Carter C, Daniels MJ, Scarpace PJ. Prolonged hyperphagia with highfat feeding contributes to exacerbated weight gain in rats with adult-onset obesity. Am J Physiol Regul Integr Comp Physiol. 2008; 295:R773-780. [PubMed: 18596107]

Jung KM, Clapper JR, Fu J, D'Agostino G, Guijarro A, Thongkham D, et al. 2-arachidonoylglycerol signaling in forebrain regulates systemic energy metabolism. Cell Metab. 2012; 15:299-310. [PubMed: 22405068]

Kadowaki T, Yamauchi T. Adiponectin and adiponectin receptors. Endocr Rev. 2005; 26:439-451. [PubMed: 15897298]

Kalra SP, Dube MG, Pu S, Xu B, Horvath TL, Kalra PS. Interacting appetite-regulating pathways in the hypothalamic regulation of body weight. Endocr Rev. 1999; 20:68-100. [PubMed: 10047974]

Kanai Y, Lee WS, You G, Brown D, Hediger MA. The human kidney low affinity Na+/glucose cotransporter SGLT2. Delineation of the major renal reabsorptive mechanism for D-glucose. J Clin Invest. 1994; 93:397-404. [PubMed: 8282810] 
Kang JG, Park CY, Kang JH, Park YW, Park SW. Randomized controlled trial to investigate the effects of a newly developed formulation of phentermine diffuse-controlled release for obesity. Diabetes Obes Metab. 2010; 12:876-882. [PubMed: 20920040]

Kanoski SE, Fortin SM, Arnold M, Grill HJ, Hayes MR. Peripheral and central GLP-1 receptor populations mediate the anorectic effects of peripherally administered GLP-1 receptor agonists, liraglutide and exendin-4. Endocrinology. 2011; 152:3103-3112. [PubMed: 21693680]

Kapur A, O'Connor-Semmes R, Hussey EK, Dobbins RL, Tao W, Hompesch M, et al. First human dose-escalation study with remogliflozin etabonate, a selective inhibitor of the sodium-glucose transporter 2 (SGLT2), in healthy subjects and in subjects with type 2 diabetes mellitus. BMC Pharmacol Toxicol. 2013; 14:26. [PubMed: 23668634]

Karamadoukis L, Shivashankar GH, Ludeman L, Williams AJ. An unusual complication of treatment with orlistat. Clin Nephrol. 2009; 71:430-432. [PubMed: 19356376]

Kawamata Y, Fujii R, Hosoya M, Harada M, Yoshida H, Miwa M, et al. A G protein-coupled receptor responsive to bile acids. J Biol Chem. 2003; 278:9435-9440. [PubMed: 12524422]

Kay K, Parise EM, Lilly N, Williams DL. Hindbrain orexin 1 receptors influence palatable food intake, operant responding for food, and food-conditioned place preference in rats. Psychopharmacology (Berl). 2014; 231:419-427. [PubMed: 23978908]

Keesey RE, Hirvonen MD. Body weight set-points: determination and adjustment. J Nutr. 1997; 127:1875S-1883S. [PubMed: 9278574]

Kelley AE. Ventral striatal control of appetitive motivation: role in ingestive behavior and rewardrelated learning. Neurosci Biobehav Rev. 2004; 27:765-776. [PubMed: 15019426]

Kelley AE, Berridge KC. The neuroscience of natural rewards: relevance to addictive drugs. J Neurosci. 2002; 22:3306-3311. [PubMed: 11978804]

Kelly E, Mundell SJ, Sava A, Roth AL, Felici A, Maltby K, et al. The opioid receptor pharmacology of GSK1521498 compared to other ligands with differential effects on compulsive reward-related behaviours. Psychopharmacology (Berl). 2015; 232:305-314. [PubMed: 24973897]

Kelly MJ, Loose MD, Ronnekleiv OK. Opioids hyperpolarize beta-endorphin neurons via mu-receptor activation of a potassium conductance. Neuroendocrinology. 1990; 52:268-275. [PubMed: 2170854]

Kenny PJ. Common cellular and molecular mechanisms in obesity and drug addiction. Nat Rev Neurosci. 2011; 12:638-651. [PubMed: 22011680]

Kernie SG, Liebl DJ, Parada LF. BDNF regulates eating behavior and locomotor activity in mice. EMBO J. 2000; 19:1290-1300. [PubMed: 10716929]

Kieffer TJ, Habener JF. The glucagon-like peptides. Endocr Rev. 1999; 20:876-913. [PubMed: 10605628]

Kievit P, Halem H, Marks DL, Dong JZ, Glavas MM, Sinnayah P, et al. Chronic treatment with a melanocortin-4 receptor agonist causes weight loss, reduces insulin resistance, and improves cardiovascular function in diet-induced obese rhesus macaques. Diabetes. 2013; 62:490-497. [PubMed: 23048186]

Kim D, MacConell L, Zhuang D, Kothare PA, Trautmann M, Fineman M, et al. Effects of once-weekly dosing of a long-acting release formulation of exenatide on glucose control and body weight in subjects with type 2 diabetes. Diabetes Care. 2007; 30:1487-1493. [PubMed: 17353504]

Kim DD, Krishnarajah J, Lillioja S, de Looze F, Marjason J, Proietto J, et al. Efficacy and safety of beloranib for weight loss in obese adults: a randomized controlled trial. Diabetes Obes Metab. 2015; 17:566-572. [PubMed: 25732625]

Kim E, Campbell S, Schueller O, Wong E, Cole B, Kuo J, et al. A small-molecule inhibitor of enterocytic microsomal triglyceride transfer protein, SLx-4090: biochemical, pharmacodynamic, pharmacokinetic, and safety profile. J Pharmacol Exp Ther. 2011; 337:775-785. [PubMed: 21406547]

Kim YM, An JJ, Jin YJ, Rhee Y, Cha BS, Lee HC, et al. Assessment of the anti-obesity effects of the TNP-470 analog, CKD-732. J Mol Endocrinol. 2007; 38:455-465. [PubMed: 17446235]

Kirkham TC, Williams CM, Fezza F, Di Marzo V. Endocannabinoid levels in rat limbic forebrain and hypothalamus in relation to fasting, feeding and satiation: stimulation of eating by 2-arachidonoyl glycerol. Br J Pharmacol. 2002; 136:550-557. [PubMed: 12055133] 
Kok P, Roelfsema F, Frolich M, van Pelt J, Meinders AE, Pijl H. Activation of dopamine D2 receptors lowers circadian leptin concentrations in obese women. J Clin Endocrinol Metab. 2006; 91:32363240. [PubMed: 16705078]

Kolterman OG, Buse JB, Fineman MS, Gaines E, Heintz S, Bicsak TA, et al. Synthetic exendin-4 (exenatide) significantly reduces postprandial and fasting plasma glucose in subjects with type 2 diabetes. J Clin Endocrinol Metab. 2003; 88:3082-3089. [PubMed: 12843147]

Koob GF, Volkow ND. Neurocircuitry of addiction. Neuropsychopharmacology. 2010; 35:217-238. [PubMed: 19710631]

Kopelman P, Groot Gde H, Rissanen A, Rossner S, Toubro S, Palmer R, et al. Weight loss, HbA1c reduction, and tolerability of cetilistat in a randomized, placebo-controlled phase 2 trial in obese diabetics: comparison with orlistat (Xenical). Obesity (Silver Spring). 2010; 18:108-115. [PubMed: 19461584]

Korbonits M, Grossman AB. Ghrelin: update on a novel hormonal system. Eur J Endocrinol. 2004; 151(Suppl 1):S67-70. [PubMed: 15339247]

Koyama S, Mori M, Kanamaru S, Sazawa T, Miyazaki A, Terai H, et al. Obesity attenuates D2 autoreceptor-mediated inhibition of putative ventral tegmental area dopaminergic neurons. Physiol Rep. 2014; 2:e12004. [PubMed: 24793981]

Krude H, Biebermann H, Luck W, Horn R, Brabant G, Gruters A. Severe early-onset obesity, adrenal insufficiency and red hair pigmentation caused by POMC mutations in humans. Nat Genet. 1998; 19:155-157. [PubMed: 9620771]

Kurth T, Gaziano JM, Berger K, Kase CS, Rexrode KM, Cook NR, et al. Body mass index and the risk of stroke in men. Arch Intern Med. 2002; 162:2557-2562. [PubMed: 12456227]

Lam DD, Przydzial MJ, Ridley SH, Yeo GS, Rochford JJ, O'Rahilly S, et al. Serotonin 5-HT2C receptor agonist promotes hypophagia via downstream activation of melanocortin 4 receptors. Endocrinology. 2008; 149:1323-1328. [PubMed: 18039773]

Launay JM, Herve P, Peoc'h K, Tournois C, Callebert J, Nebigil CG, et al. Function of the serotonin 5hydroxytryptamine 2B receptor in pulmonary hypertension. Nat Med. 2002; 8:1129-1135. [PubMed: 12244304]

Lavin JH, Wittert GA, Andrews J, Yeap B, Wishart JM, Morris HA, et al. Interaction of insulin, glucagon-like peptide 1, gastric inhibitory polypeptide, and appetite in response to intraduodenal carbohydrate. Am J Clin Nutr. 1998; 68:591-598. [PubMed: 9734735]

le Roux CW, Batterham RL, Aylwin SJ, Patterson M, Borg CM, Wynne KJ, et al. Attenuated peptide YY release in obese subjects is associated with reduced satiety. Endocrinology. 2006; 147:3-8. [PubMed: 16166213]

Lee GH, Proenca R, Montez JM, Carroll KM, Darvishzadeh JG, Lee JI, et al. Abnormal splicing of the leptin receptor in diabetic mice. Nature. 1996; 379:632-635. [PubMed: 8628397]

Lee S, Sziklas V, Andermann F, Farnham S, Risse G, Gustafson M, et al. The effects of adjunctive topiramate on cognitive function in patients with epilepsy. Epilepsia. 2003; 44:339-347. [PubMed: 12614389]

Lefebvre P, Chinetti G, Fruchart JC, Staels B. Sorting out the roles of PPAR alpha in energy metabolism and vascular homeostasis. J Clin Invest. 2006; 116:571-580. [PubMed: 16511589]

Leggio L, Schwandt ML, Oot EN, Dias AA, Ramchandani VA. Fasting-induced increase in plasma ghrelin is blunted by intravenous alcohol administration: a within-subject placebo-controlled study. Psychoneuroendocrinology. 2013; 38:3085-3091. [PubMed: 24090583]

Leggio L, Zywiak WH, Fricchione SR, Edwards SM, de la Monte SM, Swift RM, et al. Intravenous ghrelin administration increases alcohol craving in alcohol-dependent heavy drinkers: a preliminary investigation. Biol Psychiatry. 2014; 76:734-741. [PubMed: 24775991]

Leibowitz SF, Jhanwar-Uniyal M, Dvorkin B, Makman MH. Distribution of alpha-adrenergic, betaadrenergic and dopaminergic receptors in discrete hypothalamic areas of rat. Brain Res. 1982; 233:97-114. [PubMed: 6277425]

Leibowitz SF, Roossin P, Rosenn M. Chronic norepinephrine injection into the hypothalamic paraventricular nucleus produces hyperphagia and increased body weight in the rat. Pharmacol Biochem Behav. 1984; 21:801-808. [PubMed: 6514770] 
Leibowitz SF, Weiss GF, Shor-Posner G. Hypothalamic serotonin: pharmacological, biochemical, and behavioral analyses of its feeding-suppressive action. Clin Neuropharmacol. 1988; 11(Suppl 1):S51-71.

Leinninger GM, Jo YH, Leshan RL, Louis GW, Yang H, Barrera JG, et al. Leptin acts via leptin receptor-expressing lateral hypothalamic neurons to modulate the mesolimbic dopamine system and suppress feeding. Cell Metab. 2009; 10:89-98. [PubMed: 19656487]

Lent MR, Hayes SM, Wood GC, Napolitano MA, Argyropoulos G, Gerhard GS, et al. Smoking and alcohol use in gastric bypass patients. Eat Behav. 2013; 14:460-463. [PubMed: 24183136]

Levin BE, Dunn-Meynell AA. Reduced central leptin sensitivity in rats with diet-induced obesity. Am J Physiol Regul Integr Comp Physiol. 2002; 283:R941-948. [PubMed: 12228064]

Li F, Jiang C, Krausz KW, Li Y, Albert I, Hao H, et al. Microbiome remodelling leads to inhibition of intestinal farnesoid X receptor signalling and decreased obesity. Nat Commun. 2013; 4:2384. [PubMed: 24064762]

Liddle RA, Goldfine ID, Rosen MS, Taplitz RA, Williams JA. Cholecystokinin bioactivity in human plasma. Molecular forms, responses to feeding, and relationship to gallbladder contraction. J Clin Invest. 1985; 75:1144-1152. [PubMed: 2580857]

List EO, Palmer AJ, Berryman DE, Bower B, Kelder B, Kopchick JJ. Growth hormone improves body composition, fasting blood glucose, glucose tolerance and liver triacylglycerol in a mouse model of diet-induced obesity and type 2 diabetes. Diabetologia. 2009; 52:1647-1655. [PubMed: 19468705]

Lonneman DJ Jr. Rey JA, McKee BD. Phentermine/Topiramate extended-release capsules (qsymia) for weight loss. P T. 2013; 38:446-452. [PubMed: 24222976]

Lu D, Willard D, Patel IR, Kadwell S, Overton L, Kost T, et al. Agouti protein is an antagonist of the melanocyte-stimulating-hormone receptor. Nature. 1994; 371:799-802. [PubMed: 7935841]

Lutter M, Nestler EJ. Homeostatic and hedonic signals interact in the regulation of food intake. J Nutr. 2009; 139:629-632. [PubMed: 19176746]

Lutz TA, Del Prete E, Scharrer E. Reduction of food intake in rats by intraperitoneal injection of low doses of amylin. Physiol Behav. 1994; 55:891-895. [PubMed: 8022910]

MacDonald PE, De Marinis YZ, Ramracheya R, Salehi A, Ma X, Johnson PR, et al. A K ATP channeldependent pathway within alpha cells regulates glucagon release from both rodent and human islets of Langerhans. PLoS Biol. 2007; 5:e143. [PubMed: 17503968]

Macht M, Mueller J. Immediate effects of chocolate on experimentally induced mood states. Appetite. 2007; 49:667-674. [PubMed: 17597253]

Maffei M, Fei H, Lee GH, Dani C, Leroy P, Zhang Y, et al. Increased expression in adipocytes of ob RNA in mice with lesions of the hypothalamus and with mutations at the db locus. Proc Natl Acad Sci U S A. 1995; 92:6957-6960. [PubMed: 7624352]

Mahler SV, Smith KS, Berridge KC. Endocannabinoid hedonic hotspot for sensory pleasure: anandamide in nucleus accumbens shell enhances 'liking' of a sweet reward. Neuropsychopharmacology. 2007; 32:2267-2278. [PubMed: 17406653]

Mains RE, Eipper BA. Biosynthetic studies on ACTH, beta-endorphin, and alpha-melanotropin in the rat. Ann N Y Acad Sci. 1980; 343:94-110. [PubMed: 6249169]

Makishima M, Okamoto AY, Repa JJ, Tu H, Learned RM, Luk A, et al. Identification of a nuclear receptor for bile acids. Science. 1999; 284:1362-1365. [PubMed: 10334992]

Maldonado R, Valverde O, Berrendero F. Involvement of the endocannabinoid system in drug addiction. Trends Neurosci. 2006; 29:225-232. [PubMed: 16483675]

Malik S, McGlone F, Bedrossian D, Dagher A. Ghrelin modulates brain activity in areas that control appetitive behavior. Cell Metab. 2008; 7:400-409. [PubMed: 18460331]

Mansour A, Fox CA, Burke S, Meng F, Thompson RC, Akil H, et al. Mu, delta, and kappa opioid receptor mRNA expression in the rat CNS: an in situ hybridization study. J Comp Neurol. 1994; 350:412-438. [PubMed: 7884049]

Marcus JN, Aschkenasi CJ, Lee CE, Chemelli RM, Saper CB, Yanagisawa M, et al. Differential expression of orexin receptors 1 and 2 in the rat brain. J Comp Neurol. 2001; 435:6-25. [PubMed: 11370008] 
Maresz K, Pryce G, Ponomarev ED, Marsicano G, Croxford JL, Shriver LP, et al. Direct suppression of CNS autoimmune inflammation via the cannabinoid receptor CB1 on neurons and CB2 on autoreactive T cells. Nat Med. 2007; 13:492-497. [PubMed: 17401376]

Margulis AV, Mitchell AA, Gilboa SM, Werler MM, Mittleman MA, Glynn RJ, et al. Use of topiramate in pregnancy and risk of oral clefts. Am J Obstet Gynecol. 2012; 207:405, e401-407. [PubMed: 22917484]

Mark GP, Smith SE, Rada PV, Hoebel BG. An appetitively conditioned taste elicits a preferential increase in mesolimbic dopamine release. Pharmacol Biochem Behav. 1994; 48:651-660. [PubMed: 7938118]

Marks V, Morgan L, Oben J, Elliott R. Gut hormones in glucose homeostasis. Proc Nutr Soc. 1991; 50:545-552. [PubMed: 1809962]

Marks-Kaufman R, Kanarek RB. Morphine selectively influences macronutrient intake in the rat. Pharmacol Biochem Behav. 1980; 12:427-430. [PubMed: 7393941]

Marks-Kaufman R, Kanarek RB. Modifications of nutrient selection induced by naloxone in rats. Psychopharmacology (Berl). 1981; 74:321-324. [PubMed: 6794074]

Marsh DJ, Weingarth DT, Novi DE, Chen HY, Trumbauer ME, Chen AS, et al. Melanin-concentrating hormone 1 receptor-deficient mice are lean, hyperactive, and hyperphagic and have altered metabolism. Proc Natl Acad Sci U S A. 2002; 99:3240-3245. [PubMed: 11867747]

Martel P, Fantino M. Mesolimbic dopaminergic system activity as a function of food reward: a microdialysis study. Pharmacol Biochem Behav. 1996; 53:221-226. [PubMed: 8848454]

Martin CK, Redman LM, Zhang J, Sanchez M, Anderson CM, Smith SR, et al. Lorcaserin, a 5-HT(2C) receptor agonist, reduces body weight by decreasing energy intake without influencing energy expenditure. J Clin Endocrinol Metab. 2011; 96:837-845. [PubMed: 21190985]

Martin JR, Novin D. Decreased feeding in rats following hepatic-portal infusion of glucagon. Physiol Behav. 1977; 19:461-466. [PubMed: 613336]

Maruyama T, Miyamoto Y, Nakamura T, Tamai Y, Okada H, Sugiyama E, et al. Identification of membrane-type receptor for bile acids (M-BAR). Biochem Biophys Res Commun. 2002; 298:714-719. [PubMed: 12419312]

Matheny M, Shapiro A, Tumer N, Scarpace PJ. Region-specific diet-induced and leptin-induced cellular leptin resistance includes the ventral tegmental area in rats. Neuropharmacology. 2011; 60:480-487. [PubMed: 21059361]

Matson CA, Ritter RC. Long-term CCK-leptin synergy suggests a role for CCK in the regulation of body weight. Am J Physiol. 1999; 276:R1038-1045. [PubMed: 10198383]

Matsuzawa Y. Establishment of a concept of visceral fat syndrome and discovery of adiponectin. Proc Jpn Acad Ser B Phys Biol Sci. 2010; 86:131-141.

Mayo KE, Miller LJ, Bataille D, Dalle S, Goke B, Thorens B, et al. International Union of Pharmacology. XXXV. The glucagon receptor family. Pharmacol Rev. 2003; 55:167-194. [PubMed: 12615957]

McElroy SL, Hudson JI, Capece JA, Beyers K, Fisher AC, Rosenthal NR, et al. Topiramate for the treatment of binge eating disorder associated with obesity: a placebo-controlled study. Biol Psychiatry. 2007; 61:1039-1048. [PubMed: 17258690]

McNeely W, Benfield P. Orlistat. Drugs. 1998; 56:241-249. discussion 250. [PubMed: 9711448]

Mebel DM, Wong JC, Dong YJ, Borgland SL. Insulin in the ventral tegmental area reduces hedonic feeding and suppresses dopamine concentration via increased reuptake. Eur J Neurosci. 2012; 36:2336-2346. [PubMed: 22712725]

Melis M, Pistis M, Perra S, Muntoni AL, Pillolla G, Gessa GL. Endocannabinoids mediate presynaptic inhibition of glutamatergic transmission in rat ventral tegmental area dopamine neurons through activation of CB1 receptors. J Neurosci. 2004; 24:53-62. [PubMed: 14715937]

Melis T, Succu S, Sanna F, Boi A, Argiolas A, Melis MR. The cannabinoid antagonist SR 141716A (Rimonabant) reduces the increase of extra-cellular dopamine release in the rat nucleus accumbens induced by a novel high palatable food. Neurosci Lett. 2007; 419:231-235. [PubMed: 17462824]

Menendez JA, Atrens DM. Insulin and the paraventricular hypothalamus: modulation of energy balance. Brain Res. 1991; 555:193-201. [PubMed: 1933333] 
Merchenthaler I, Lane M, Shughrue P. Distribution of pre-pro-glucagon and glucagon-like peptide-1 receptor messenger RNAs in the rat central nervous system. J Comp Neurol. 1999; 403:261-280. [PubMed: 9886047]

Meye FJ, Trezza V, Vanderschuren LJ, Ramakers GM, Adan RA. Neutral antagonism at the cannabinoid 1 receptor: a safer treatment for obesity. Mol Psychiatry. 2013; 18:1294-1301. [PubMed: 23070073]

Midtvedt T. Microbial bile acid transformation. Am J Clin Nutr. 1974; 27:1341-1347. [PubMed: 4217103]

Miller MW, Duhl DM, Vrieling H, Cordes SP, Ollmann MM, Winkes BM, et al. Cloning of the mouse agouti gene predicts a secreted protein ubiquitously expressed in mice carrying the lethal yellow mutation. Genes Dev. 1993; 7:454-467. [PubMed: 8449404]

Mindell S, Smith GP, Greenberg D. Corn oil and mineral oil stimulate sham feeding in rats. Physiol Behav. 1990; 48:283-287. [PubMed: 2255732]

Mokdad AH, Bowman BA, Ford ES, Vinicor F, Marks JS, Koplan JP. The continuing epidemics of obesity and diabetes in the United States. JAMA. 2001; 286:1195-1200. [PubMed: 11559264]

Moller N, Jorgensen JO. Effects of growth hormone on glucose, lipid, and protein metabolism in human subjects. Endocr Rev. 2009; 30:152-177. [PubMed: 19240267]

Mondal MS, Date Y, Yamaguchi H, Toshinai K, Tsuruta T, Kangawa K, et al. Identification of ghrelin and its receptor in neurons of the rat arcuate nucleus. Regul Pept. 2005; 126:55-59. [PubMed: 15620414]

Montani JP, Antic V, Yang Z, Dulloo A. Pathways from obesity to hypertension: from the perspective of a vicious triangle. Int J Obes Relat Metab Disord. 2002; 26(Suppl 2):S28-38. [PubMed: 12174326]

Montgomery IA, Irwin N, Flatt PR. Beneficial effects of (pGlu-Gln)-CCK-8 on energy intake and metabolism in high fat fed mice are associated with alterations of hypothalamic gene expression. Horm Metab Res. 2013; 45:471-473. [PubMed: 23315994]

Moran TH, Ameglio PJ, Schwartz GJ, McHugh PR. Blockade of type A, not type B, CCK receptors attenuates satiety actions of exogenous and endogenous CCK. Am J Physiol. 1992; 262:R46-50. [PubMed: 1733339]

Moreau, RA. Corn oil. In: Gunstone, F., editor. Vegetable Oils in Food Technology: Composition, Properties and Uses. Wiley-Blackwell; New York: 2011. p. 273-289.

Morrison CD, Berthoud HR. Neurobiology of nutrition and obesity. Nutr Rev. 2007; 65:517-534. [PubMed: 18236691]

Munro JF, MacCuish AC, Wilson EM, Duncan LJ. Comparison of continuous and intermittent anorectic therapy in obesity. Br Med J. 1968; 1:352-354. [PubMed: 15508204]

Myers MG, Cowley MA, Munzberg H. Mechanisms of leptin action and leptin resistance. Annu Rev Physiol. 2008; 70:537-556. [PubMed: 17937601]

Nair KS. Hyperglucagonemia increases resting metabolic rate in man during insulin deficiency. J Clin Endocrinol Metab. 1987; 64:896-901. [PubMed: 2881943]

Nam SY, Kim KR, Cha BS, Song YD, Lim SK, Lee HC, et al. Low-dose growth hormone treatment combined with diet restriction decreases insulin resistance by reducing visceral fat and increasing muscle mass in obese type 2 diabetic patients. Int J Obes Relat Metab Disord. 2001; 25:11011107. [PubMed: 11477493]

Narayanaswami V, Thompson AC, Cassis LA, Bardo MT, Dwoskin LP. Diet-induced obesity: dopamine transporter function, impulsivity and motivation. Int J Obes (Lond). 2013; 37:10951103. [PubMed: 23164701]

Naslund E, Bogefors J, Skogar S, Gryback P, Jacobsson H, Holst JJ, et al. GLP-1 slows solid gastric emptying and inhibits insulin, glucagon, and PYY release in humans. Am J Physiol. 1999; 277:R910-916. [PubMed: 10484511]

Nathan PJ, O'Neill BV, Bush MA, Koch A, Tao WX, Maltby K, et al. Opioid receptor modulation of hedonic taste preference and food intake: a single-dose safety, pharmacokinetic, and pharmacodynamic investigation with GSK1521498, a novel mu-opioid receptor inverse agonist. J Clin Pharmacol. 2012; 52:464-474. [PubMed: 21610207] 
Nathan PJ, O'Neill BV, Napolitano A, Bullmore ET. Neuropsychiatric adverse effects of centrally acting antiobesity drugs. CNS Neurosci Ther. 2011; 17:490-505. [PubMed: 21951371]

National Health and Examination Survey. 2010. http://www.cdc.gov/nchs/nhanes.htm

Navarrete F, Rodriguez-Arias M, Martin-Garcia E, Navarro D, Garcia-Gutierrez MS, Aguilar MA, et al. Role of CB2 cannabinoid receptors in the rewarding, reinforcing, and physical effects of nicotine. Neuropsychopharmacology. 2013; 38:2515-2524. [PubMed: 23817165]

NCI. 2012. http://www.cancer.gov/cancertopics/factsheet/Risk/obesity

Nielsen C, Gormsen LC, Jessen N, Pedersen SB, Moller N, Lund S, et al. Growth hormone signaling in vivo in human muscle and adipose tissue: impact of insulin, substrate background, and growth hormone receptor blockade. J Clin Endocrinol Metab. 2008; 93:2842-2850. [PubMed: 18460563]

Ng M, Fleming T, Robinson M, Thomson B, Graetz N, Margono C, et al. Global, regional, and national prevalence of overweight and obesity in children and adults during 1980-2013: a systematic analysis for the Global Burden of Disease Study 2013. Lancet. 2014; 384:766-781. [PubMed: 24880830]

Nogueiras R, Romero-Pico A, Vazquez MJ, Novelle MG, Lopez M, Dieguez C. The opioid system and food intake: homeostatic and hedonic mechanisms. Obes Facts. 2012; 5:196-207. [PubMed: 22647302]

Obici S, Feng Z, Karkanias G, Baskin DG, Rossetti L. Decreasing hypothalamic insulin receptors causes hyperphagia and insulin resistance in rats. Nat Neurosci. 2002; 5:566-572. [PubMed: 12021765]

O'Dell LE, Koob GF. 'Nicotine deprivation effect' in rats with intermittent 23-hour access to intravenous nicotine self-administration. Pharmacol Biochem Behav. 2007; 86:346-353. [PubMed: 17292952]

O'Dell LE, Natividad LA, Pipkin JA, Roman F, Torres I, Jurado J, et al. Enhanced nicotine selfadministration and suppressed dopaminergic systems in a rat model of diabetes. Addict Biol. 2014; 19:1006-1019. [PubMed: 23834715]

Oltmans GA. Norepinephrine and dopamine levels in hypothalamic nuclei of the genetically obese mouse (ob/ob). Brain Res. 1983; 273:369-373. [PubMed: 6616244]

Onaga T, Zabielski R, Kato S. Multiple regulation of peptide YY secretion in the digestive tract. Peptides. 2002; 23:279-290. [PubMed: 11825643]

Onaivi ES, Ishiguro H, Gong JP, Patel S, Perchuk A, Meozzi PA, et al. Discovery of the presence and functional expression of cannabinoid CB2 receptors in brain. Ann N Y Acad Sci. 2006; 1074:514-536. [PubMed: 17105950]

O'Neil PM, Smith SR, Weissman NJ, Fidler MC, Sanchez M, Zhang J, et al. Randomized placebocontrolled clinical trial of lorcaserin for weight loss in type 2 diabetes mellitus: the BLOOM-DM study. Obesity (Silver Spring). 2012; 20:1426-1436. [PubMed: 22421927]

Opland DM, Leinninger GM, Myers MG Jr. Modulation of the mesolimbic dopamine system by leptin. Brain Res. 2010; 1350:65-70. [PubMed: 20417193]

Osei-Hyiaman D, Depetrillo M, Harvey-White J, Bannon AW, Cravatt BF, Kuhar MJ, et al. Cocaineand amphetamine-related transcript is involved in the orexigenic effect of endogenous anandamide. Neuroendocrinology. 2005a; 81:273-282. [PubMed: 16131814]

Osei-Hyiaman D, DePetrillo M, Pacher P, Liu J, Radaeva S, Batkai S, et al. Endocannabinoid activation at hepatic $\mathrm{CB} 1$ receptors stimulates fatty acid synthesis and contributes to diet-induced obesity. J Clin Invest. 2005b; 115:1298-1305. [PubMed: 15864349]

Osei-Hyiaman D, Liu J, Zhou L, Godlewski G, Harvey-White J, Jeong WI, et al. Hepatic CB1 receptor is required for development of diet-induced steatosis, dyslipidemia, and insulin and leptin resistance in mice. J Clin Invest. 2008; 118:3160-3169. [PubMed: 18677409]

Oveisi F, Gaetani S, Eng KT, Piomelli D. Oleoylethanolamide inhibits food intake in free-feeding rats after oral administration. Pharmacol Res. 2004; 49:461-466. [PubMed: 14998556]

Padwal RS, Majumdar SR. Drug treatments for obesity: orlistat, sibutramine, and rimonabant. Lancet. 2007; 369:71-77. [PubMed: 17208644] 
Palkovits M. Interconnections between the neuroendocrine hypothalamus and the central autonomic system. Geoffrey Harris Memorial Lecture, Kitakyushu, Japan, October 1998. Front Neuroendocrinol. 1999; 20:270-295. [PubMed: 10569279]

Palmiter RD. Is dopamine a physiologically relevant mediator of feeding behavior? Trends Neurosci. 2007; 30:375-381. [PubMed: 17604133]

Parker JA, Bloom SR. Hypothalamic neuropeptides and the regulation of appetite. [Review]. Neuropharmacology. 2012; 63(1):18-30. [PubMed: 22369786]

Papaleo F, Kieffer BL, Tabarin A, Contarino A. Decreased motivation to eat in mu-opioid receptordeficient mice. Eur J Neurosci. 2007; 25:3398-3405. [PubMed: 17553008]

Parseus A, Sommer N, Sommer F, Caesar R, Molinaro A, Stahlman M, et al. Microbiota-induced obesity requires farnesoid X receptor. Gut. 2016

Pecina S, Berridge KC. Hedonic hot spot in nucleus accumbens shell: where do mu-opioids cause increased hedonic impact of sweetness? J Neurosci. 2005; 25:11777-11786. [PubMed: 16354936]

Pecina S, Cagniard B, Berridge KC, Aldridge JW, Zhuang X. Hyperdopaminergic mutant mice have higher "wanting" but not "liking" for sweet rewards. J Neurosci. 2003; 23:9395-9402. [PubMed: 14561867]

Pelchat ML, Johnson A, Chan R, Valdez J, Ragland JD. Images of desire: food-craving activation during fMRI. Neuroimage. 2004; 23:1486-1493. [PubMed: 15589112]

Penick SB, Hinkle LE Jr. Depression of food intake induced in healthy subjects by glucagon. N Engl J Med. 1961; 264:893-897. [PubMed: 13734109]

Penney NC, Kinross J, Newton RC, Purkayastha S. The role of bile acids in reducing the metabolic complications of obesity after bariatric surgery: a systematic review. Int J Obes (Lond). 2015; 39:1565-1574. [PubMed: 26081915]

Peruzzo B, Pastor FE, Blazquez JL, Schobitz K, Pelaez B, Amat P, et al. A second look at the barriers of the medial basal hypothalamus. Exp Brain Res. 2000; 132:10-26. [PubMed: 10836632]

Peterson CB, Mitchell JE. Psychosocial and pharmacological treatment of eating disorders: a review of research findings. J Clin Psychol. 1999; 55:685-697. [PubMed: 10445860]

Petry NM, Barry D, Pietrzak RH, Wagner JA. Overweight and obesity are associated with psychiatric disorders: results from the National Epidemiologic Survey on Alcohol and Related Conditions. Psychosom Med. 2008; 70:288-297. [PubMed: 18378873]

Peyron C, Tighe DK, van den Pol AN, de Lecea L, Heller HC, Sutcliffe JG, et al. Neurons containing hypocretin (orexin) project to multiple neuronal systems. J Neurosci. 1998; 18:9996-10015. [PubMed: 9822755]

Piccoli L, Micioni Di Bonaventura MV, Cifani C, Costantini VJ, Massagrande M, Montanari D, et al. Role of orexin-1 receptor mechanisms on compulsive food consumption in a model of binge eating in female rats. Neuropsychopharmacology. 2012; 37:1999-2011. [PubMed: 22569505]

Pijl H, Ohashi S, Matsuda M, Miyazaki Y, Mahankali A, Kumar V, et al. Bromocriptine: a novel approach to the treatment of type 2 diabetes. Diabetes Care. 2000; 23:1154-1161. [PubMed: 10937514]

Piomelli D. The endocannabinoid system: a drug discovery perspective. Curr Opin Investig Drugs. 2005; 6:672-679.

Piomelli D. A fatty gut feeling. Trends Endocrinol Metab. 2013; 24:332-341. [PubMed: 23567058]

Pischon T, Girman CJ, Rifai N, Hotamisligil GS, Rimm EB. Association between dietary factors and plasma adiponectin concentrations in men. Am J Clin Nutr. 2005; 81:780-786. [PubMed: 15817852]

Pissios P. Animals models of MCH function and what they can tell us about its role in energy balance. Peptides. 2009; 30:2040-2044. [PubMed: 19447150]

Pissios P, Bradley RL, Maratos-Flier E. Expanding the scales: The multiple roles of MCH in regulating energy balance and other biological functions. Endocr Rev. 2006; 27:606-620. [PubMed: 16788162]

Pi-Sunyer X, Obesity S, Prediabetes I. Liraglutide in Weight Management. N Engl J Med. 2015; 373:1781-1782. 
Pocai A. Action and therapeutic potential of oxyntomodulin. Mol Metab. 2014; 3:241-251. [PubMed: 24749050]

Pocai A, Carrington PE, Adams JR, Wright M, Eiermann G, Zhu L, et al. Glucagon-like peptide 1/ glucagon receptor dual agonism reverses obesity in mice. Diabetes. 2009; 58:2258-2266. [PubMed: 19602537]

Polonsky KS, Klein S. Gastric banding to treat obesity: band-aid or breakthrough? Nat Clin Pract Endocrinol Metab. 2008; 4:421. [PubMed: 18648393]

Polston JE, Pritchett CE, Tomasko JM, Rogers AM, Leggio L, Thanos PK, et al. Roux-en-Y gastric bypass increases intravenous ethanol self-administration in dietary obese rats. PLoS One. 2013; 8:e83741. [PubMed: 24391816]

Poole DP, Godfrey C, Cattaruzza F, Cottrell GS, Kirkland JG, Pelayo JC, et al. Expression and function of the bile acid receptor GpBAR1 (TGR5) in the murine enteric nervous system. Neurogastroenterol Motil. 2010; 22:814-825. e227-818. [PubMed: 20236244]

Porte D Jr. Baskin DG, Schwartz MW. Leptin and insulin action in the central nervous system. Nutr Rev. 2002; 60:S20-29. discussion S68-84, 85-27. [PubMed: 12403080]

Prawitt J, Abdelkarim M, Stroeve JH, Popescu I, Duez H, Velagapudi VR, et al. Farnesoid X receptor deficiency improves glucose homeostasis in mouse models of obesity. Diabetes. 2011; 60:18611871. [PubMed: 21593203]

Provensi G, Coccurello R, Umehara H, Munari L, Giacovazzo G, Galeotti N, et al. Satiety factor oleoylethanolamide recruits the brain histaminergic system to inhibit food intake. Proc Natl Acad Sci U S A. 2014; 111:11527-11532. [PubMed: 25049422]

Qi Y, Takahashi N, Hileman SM, Patel HR, Berg AH, Pajvani UB, et al. Adiponectin acts in the brain to decrease body weight. Nat Med. 2004; 10:524-529. [PubMed: 15077108]

Qu D, Ludwig DS, Gammeltoft S, Piper M, Pelleymounter MA, Cullen MJ, et al. A role for melaninconcentrating hormone in the central regulation of feeding behaviour. Nature. 1996; 380:243247. [PubMed: 8637571]

Quarta C, Bellocchio L, Mancini G, Mazza R, Cervino C, Braulke LJ, et al. CB(1) signaling in forebrain and sympathetic neurons is a key determinant of endocannabinoid actions on energy balance. Cell Metab. 2010; 11:273-285. [PubMed: 20374960]

Ravinet Trillou C, Delgorge C, Menet C, Arnone M, Soubrie P. CB1 cannabinoid receptor knockout in mice leads to leanness, resistance to diet-induced obesity and enhanced leptin sensitivity. Int J Obes Relat Metab Disord. 2004; 28:640-648. [PubMed: 14770190]

Ravussin E, Smith SR, Mitchell JA, Shringarpure R, Shan K, Maier H, et al. Enhanced weight loss with pramlintide/metreleptin: an integrated neurohormonal approach to obesity pharmacotherapy. Obesity (Silver Spring). 2009; 17:1736-1743. [PubMed: 19521351]

Read NW. Role of gastrointestinal factors in hunger and satiety in man. Proc Nutr Soc. 1992; 51:7-11. [PubMed: 1508932]

Rehfeld JF, Sun G, Christensen T, Hillingso JG. The predominant cholecystokinin in human plasma and intestine is cholecystokinin-33. J Clin Endocrinol Metab. 2001; 86:251-258. [PubMed: 11232009]

Richard D, Ferland J, Lalonde J, Samson P, Deshaies Y. Influence of topiramate in the regulation of energy balance. Nutrition. 2000; 16:961-966. [PubMed: 11054602]

Richard JE, Anderberg RH, Goteson A, Gribble FM, Reimann F, Skibicka KP. Activation of the GLP-1 receptors in the nucleus of the solitary tract reduces food reward behavior and targets the mesolimbic system. PLoS One. 2015; 10:e0119034. [PubMed: 25793511]

Richards JK, Simms JA, Steensland P, Taha SA, Borgland SL, Bonci A, et al. Inhibition of orexin-1/ hypocretin-1 receptors inhibits yohimbine-induced reinstatement of ethanol and sucrose seeking in Long-Evans rats. Psychopharmacology (Berl). 2008; 199:109-117. [PubMed: 18470506]

Richardson JR, Pipkin JA, O'Dell LE, Nazarian A. Insulin resistant rats display enhanced rewarding effects of nicotine. Drug Alcohol Depend. 2014; 140:205-207. [PubMed: 24774962]

Richardson NR, Roberts DC. Progressive ratio schedules in drug self-administration studies in rats: a method to evaluate reinforcing efficacy. J Neurosci Methods. 1996; 66:1-11. [PubMed: 8794935] 
Riegel AC, Lupica CR. Independent presynaptic and postsynaptic mechanisms regulate endocannabinoid signaling at multiple synapses in the ventral tegmental area. J Neurosci. 2004; 24:11070-11078. [PubMed: 15590923]

Rodgers RJ, Tschop MH, Wilding JP. Anti-obesity drugs: past, present and future. Dis Model Mech. 2012; 5:621-626. [PubMed: 22915024]

Rodriguez de Fonseca F, Navarro M, Gomez R, Escuredo L, Nava F, Fu J, et al. An anorexic lipid mediator regulated by feeding. Nature. 2001; 414:209-212. [PubMed: 11700558]

Roevens P, Heeres J, Meerpoel L, Dupont A, Borghys H, Lammens L, et al. Hypolipidemic effects of R103757, a potent stereoselective inhibitor of microsomal triglyceride transfer protein (MTP). Atherosclerosis. 1999; 144(Suppl 1):38.

Rolls ET. Taste, olfactory and food texture reward processing in the brain and obesity. Int J Obes (Lond). 2011; 35:550-561. [PubMed: 20680018]

Rosenstock J, Klaff LJ, Schwartz S, Northrup J, Holcombe JH, Wilhelm K, et al. Effects of exenatide and lifestyle modification on body weight and glucose tolerance in obese subjects with and without pre-diabetes. Diabetes Care. 2010; 33:1173-1175. [PubMed: 20332357]

Rossi M, Kim MS, Morgan DG, Small CJ, Edwards CM, Sunter D, et al. A C-terminal fragment of Agouti-related protein increases feeding and antagonizes the effect of alpha-melanocyte stimulating hormone in vivo. Endocrinology. 1998; 139:4428-4431. [PubMed: 9751529]

Roth JD, Roland BL, Cole RL, Trevaskis JL, Weyer C, Koda JE, et al. Leptin responsiveness restored by amylin agonism in diet-induced obesity: evidence from nonclinical and clinical studies. Proc Natl Acad Sci U S A. 2008; 105:7257-7262. [PubMed: 18458326]

Rushing PA, Hagan MM, Seeley RJ, Lutz TA, D'Alessio DA, Air EL, et al. Inhibition of central amylin signaling increases food intake and body adiposity in rats. Endocrinology. 2001; 142:5035. [PubMed: 11606473]

Sadry SA, Drucker DJ. Emerging combinatorial hormone therapies for the treatment of obesity and T2DM. Nat Rev Endocrinol. 2013; 9:425-433. [PubMed: 23478327]

Sainsbury A, Schwarzer C, Couzens M, Fetissov S, Furtinger S, Jenkins A, et al. Important role of hypothalamic Y2 receptors in body weight regulation revealed in conditional knockout mice. Proc Natl Acad Sci U S A. 2002; 99:8938-8943. [PubMed: 12072562]

Sakurai T, Amemiya A, Ishii M, Matsuzaki I, Chemelli RM, Tanaka H, et al. Orexins and orexin receptors: a family of hypothalamic neuropeptides and $\mathrm{G}$ protein-coupled receptors that regulate feeding behavior. Cell. 1998; 92:573-585. [PubMed: 9491897]

Sam AH, Troke RC, Tan TM, Bewick GA. The role of the gut/brain axis in modulating food intake. Neuropharmacology. 2012; 63(1):46-56. [PubMed: 22037149]

Sanchis-Segura C, Cline BH, Marsicano G, Lutz B, Spanagel R. Reduced sensitivity to reward in CB1 knockout mice. Psychopharmacology (Berl). 2004; 176:223-232. [PubMed: 15083252]

Sanger GJ, Hellstrom PM, Naslund E. The hungry stomach: physiology, disease, and drug development opportunities. Front Pharmacol. 2010; 1:145. [PubMed: 21927604]

Saper CB, Chou TC, Elmquist JK. The need to feed: homeostatic and hedonic control of eating. Neuron. 2002; 36:199-211. [PubMed: 12383777]

Sarica K, Akarsu E, Erturhan S, Yagci F, Aktaran S, Altay B. Evaluation of urinary oxalate levels in patients receiving gastrointestinal lipase inhibitor. Obesity (Silver Spring). 2008; 16:1579-1584. [PubMed: 18451780]

Sarkar S, Lechan RM. Central administration of neuropeptide Y reduces alpha-melanocyte-stimulating hormone-induced cyclic adenosine 5'-monophosphate response element binding protein (CREB) phosphorylation in pro-thyrotropin-releasing hormone neurons and increases CREB phosphorylation in corticotropin-releasing hormone neurons in the hypothalamic paraventricular nucleus. Endocrinology. 2003; 144:281-291. [PubMed: 12488356]

Sayin SI, Wahlstrom A, Felin J, Jantti S, Marschall HU, Bamberg K, et al. Gut microbiota regulates bile acid metabolism by reducing the levels of tauro-beta-muricholic acid, a naturally occurring FXR antagonist. Cell Metab. 2013; 17:225-235. [PubMed: 23395169]

Scarpace PJ, Matheny M, Tumer N, Cheng KY, Zhang Y. Leptin resistance exacerbates diet-induced obesity and is associated with diminished maximal leptin signalling capacity in rats. Diabetologia. 2005; 48:1075-1083. [PubMed: 15864530] 
Scarpace PJ, Zhang Y. Leptin resistance: a prediposing factor for diet-induced obesity. Am J Physiol Regul Integr Comp Physiol. 2009; 296:R493-500. [PubMed: 19091915]

Scheen AJ. Sibutramine on cardiovascular outcome. Diabetes Care. 2011; 34(Suppl 2):S114-119. [PubMed: 21525441]

Schur EA, Kleinhans NM, Goldberg J, Buchwald D, Schwartz MW, Maravilla K. Activation in brain energy regulation and reward centers by food cues varies with choice of visual stimulus. Int $\mathrm{J}$ Obes (Lond). 2009; 33:653-661. [PubMed: 19365394]

Schwartz GJ, Fu J, Astarita G, Li X, Gaetani S, Campolongo P, et al. The lipid messenger OEA links dietary fat intake to satiety. Cell Metab. 2008; 8:281-288. [PubMed: 18840358]

Schwartz MW, Woods SC, Porte D Jr. Seeley RJ, Baskin DG. Central nervous system control of food intake. Nature. 2000; 404:661-671. [PubMed: 10766253]

Sclafani A, Bodnar RJ, Delamater AR. Pharmacology of food conditioned preferences. Appetite. 1998; 31:406. [PubMed: 9920694]

Scott KA, Moran TH. The GLP-1 agonist exendin-4 reduces food intake in nonhuman primates through changes in meal size. Am J Physiol Regul Integr Comp Physiol. 2007; 293:R983-987. [PubMed: 17581835]

Sears RM, Liu RJ, Narayanan NS, Sharf R, Yeckel MF, Laubach M, et al. Regulation of nucleus accumbens activity by the hypothalamic neuropeptide melanin-concentrating hormone. $\mathrm{J}$ Neurosci. 2010; 30:8263-8273. [PubMed: 20554878]

Self DW, Nestler EJ. Relapse to drug-seeking: neural and molecular mechanisms. Drug Alcohol Depend. 1998; 51:49-60. [PubMed: 9716929]

Selvakumar P, Pasha MK, Ashakumary L, Dimmock JR, Sharma RK. Myristoyl-CoA:protein Nmyristoyltransferase: a novel molecular approach for cancer therapy (Review). Int J Mol Med. 2002; 10:493-500. [PubMed: 12239600]

Shaham Y, Shalev U, Lu L, De Wit H, Stewart J. The reinstatement model of drug relapse: history, methodology and major findings. Psychopharmacology (Berl). 2003; 168:3-20. [PubMed: 12402102]

Sherwood A, Holland PC, Adamantidis A, Johnson AW. Deletion of Melanin Concentrating Hormone Receptor-1 disrupts overeating in the presence of food cues. Physiol Behav. 2015; 152:402-407. [PubMed: 26048303]

Shimano H, Horton JD, Hammer RE, Shimomura I, Brown MS, Goldstein JL. Overproduction of cholesterol and fatty acids causes massive liver enlargement in transgenic mice expressing truncated SREBP-1a. J Clin Invest. 1996; 98:1575-1584. [PubMed: 8833906]

Shinohara M, Mizushima H, Hirano M, Shioe K, Nakazawa M, Hiejima Y, et al. Eating disorders with binge-eating behaviour are associated with the s allele of the 3'-UTR VNTR polymorphism of the dopamine transporter gene. J Psychiatry Neurosci. 2004; 29:134-137. [PubMed: 15069467]

Shippenberg, TS., Koob, GF. Recent advances in animal models of drug addiction and alcoholism. In: Davis, KL.Charney, D.Coyle, JT., Nemeroff, C., editors. Neuropsychopharmacology: The Fifth Generation of Progress. Lippincott Williams and Wilkins; Philadelphia: 2002. p. 1381-1397.

Shram MJ, Schoedel KA, Bartlett C, Shazer RL, Anderson CM, Sellers EM. Evaluation of the abuse potential of lorcaserin, a serotonin $2 \mathrm{C}(5-\mathrm{HT} 2 \mathrm{C})$ receptor agonist, in recreational polydrug users. Clin Pharmacol Ther. 2011; 89:683-692. [PubMed: 21412231]

Siebenhofer A, Horvath K, Jeitler K, Berghold A, Stich AK, Matyas E, et al. Long-term effects of weight-reducing drugs in hypertensive patients. Cochrane Database Syst Rev. 2009:CD007654. [PubMed: 19588440]

Simmons WK, Martin A, Barsalou LW. Pictures of appetizing foods activate gustatory cortices for taste and reward. Cereb Cortex. 2005; 15:1602-1608. [PubMed: 15703257]

Simon JR, Bare DJ, Ghetti B, Richter JA. A possible role for tyrosine kinases in the regulation of the neuronal dopamine transporter in mouse striatum. Neurosci Lett. 1997; 224:201-205. [PubMed: 9131671]

Sin N, Meng L, Wang MQ, Wen JJ, Bornmann WG, Crews CM. The anti-angiogenic agent fumagillin covalently binds and inhibits the methionine aminopeptidase, MetAP-2. Proc Natl Acad Sci U S A. 1997; 94:6099-6103. [PubMed: 9177176] 
Sinal CJ, Tohkin M, Miyata M, Ward JM, Lambert G, Gonzalez FJ. Targeted disruption of the nuclear receptor FXR/BAR impairs bile acid and lipid homeostasis. Cell. 2000; 102:731-744. [PubMed: 11030617]

Sinnayah, P., Wallingford, N., Evans, A., Cowley, MA. Bupropion and naltrexone interact synergistically to decrease food intake in mice. Presented at the North American association for the study of obesity annual scientific meeting; New Orleans, LA. 2007.

Sjostrom L, Rissanen A, Andersen T, Boldrin M, Golay A, Koppeschaar HP, et al. Randomised placebo-controlled trial of orlistat for weight loss and prevention of weight regain in obese patients. European Multicentre Orlistat Study Group. Lancet. 1998; 352:167-172. [PubMed: 9683204]

Skibicka KP. The central GLP-1: implications for food and drug reward. Front Neurosci. 2013; 7:181. [PubMed: 24133407]

Skibicka KP, Hansson C, Alvarez-Crespo M, Friberg PA, Dickson SL. Ghrelin directly targets the ventral tegmental area to increase food motivation. Neuroscience. 2011; 180:129-137. [PubMed: 21335062]

Skibicka KP, Hansson C, Egecioglu E, Dickson SL. Role of ghrelin in food reward: impact of ghrelin on sucrose self-administration and mesolimbic dopamine and acetylcholine receptor gene expression. Addict Biol. 2012a; 17:95-107. [PubMed: 21309956]

Skibicka KP, Shirazi RH, Hansson C, Dickson SL. Ghrelin interacts with neuropeptide Y Y1 and opioid receptors to increase food reward. Endocrinology. 2012b; 153:1194-1205. [PubMed: 22210742]

Skibicka KP, Shirazi RH, Rabasa-Papio C, Alvarez-Crespo M, Neuber C, Vogel H, et al. Divergent circuitry underlying food reward and intake effects of ghrelin: dopaminergic VTA-accumbens projection mediates ghrelin's effect on food reward but not food intake. Neuropharmacology. 2013; 73:274-283. [PubMed: 23770258]

Small DM, Jones-Gotman M, Dagher A. Feeding-induced dopamine release in dorsal striatum correlates with meal pleasantness ratings in healthy human volunteers. Neuroimage. 2003; 19:1709-1715. [PubMed: 12948725]

Small DM, Zatorre RJ, Dagher A, Evans AC, Jones-Gotman M. Changes in brain activity related to eating chocolate: from pleasure to aversion. Brain. 2001; 124:1720-1733. [PubMed: 11522575]

Smith SC Jr. Benjamin EJ, Bonow RO, Braun LT, Creager MA, Franklin BA, et al. AHA/ACCF Secondary Prevention and Risk Reduction Therapy for Patients with Coronary and other Atherosclerotic Vascular Disease: 2011 update: a guideline from the American Heart Association and American College of Cardiology Foundation. Circulation. 2011; 124:2458-2473. [PubMed: 22052934]

Smith SR, Weissman NJ, Anderson CM, Sanchez M, Chuang E, Stubbe S, et al. Multicenter, placebocontrolled trial of lorcaserin for weight management. N Engl J Med. 2010; 363:245-256. [PubMed: 20647200]

Sohn JW, Elmquist JK, Williams KW. Neuronal circuits that regulate feeding behavior and metabolism. Trends Neurosci. 2013; 36:504-512. [PubMed: 23790727]

Sohn JW. Network of hypothalamic neurons that control appetite. BMB Rep. 2015; 48(4):229-233. [PubMed: 25560696]

Spiegelman BM, Flier JS. Obesity and the regulation of energy balance. Cell. 2001; 104:531-543. [PubMed: 11239410]

Stanley BG, Kyrkouli SE, Lampert S, Leibowitz SF. Neuropeptide Y chronically injected into the hypothalamus: a powerful neurochemical inducer of hyperphagia and obesity. Peptides. 1986; 7:1189-1192. [PubMed: 3470711]

Stanley S, Wynne K, McGowan B, Bloom S. Hormonal regulation of food intake. Physiol Rev. 2005; 85:1131-1158. [PubMed: 16183909]

Steinert RE, Poller B, Castelli MC, Drewe J, Beglinger C. Oral administration of glucagon-like peptide 1 or peptide YY 3-36 affects food intake in healthy male subjects. Am J Clin Nutr. 2010; 92:810817. [PubMed: 20720259]

Sternbach H. The serotonin syndrome. Am J Psychiatry. 1991; 148:705-713. [PubMed: 2035713] 
Stice E, Spoor S, Bohon C, Veldhuizen MG, Small DM. Relation of reward from food intake and anticipated food intake to obesity: a functional magnetic resonance imaging study. J Abnorm Psychol. 2008; 117:924-935. [PubMed: 19025237]

Sulzer D, Sonders MS, Poulsen NW, Galli A. Mechanisms of neurotransmitter release by amphetamines: a review. Prog Neurobiol. 2005; 75:406-433. [PubMed: 15955613]

Sweeting AN, Tabet E., Caterson, I. D. Markovic TP. Management of obesity and cardiometabolic risk - role of phentermine/extended release topiramate. Diabetes Metab Syndr Obes. 2014; 7:35-44. [PubMed: 24550678]

Szabo B, Siemes S, Wallmichrath I. Inhibition of GABAergic neurotransmission in the ventral tegmental area by cannabinoids. Eur J Neurosci. 2002; 15:2057-2061. [PubMed: 12099913]

Tabarin A, Diz-Chaves Y, Carmona Mdel C, Catargi B, Zorrilla EP, Roberts AJ, et al. Resistance to diet-induced obesity in mu-opioid receptor-deficient mice: evidence for a "thrifty gene". Diabetes. 2005; 54:3510-3516. [PubMed: 16306369]

Taha C, Klip A. The insulin signaling pathway. J Membr Biol. 1999; 169:1-12. [PubMed: 10227847]

Tan CP, Sano H, Iwaasa H, Pan J, Sailer AW, Hreniuk DL, et al. Melanin-concentrating hormone receptor subtypes 1 and 2: species-specific gene expression. Genomics. 2002; 79:785-792. [PubMed: 12036292]

Tan TM, Field BC, McCullough KA, Troke RC, Chambers ES, Salem V, et al. Coadministration of glucagon-like peptide-1 during glucagon infusion in humans results in increased energy expenditure and amelioration of hyperglycemia. Diabetes. 2013; 62:1131-1138. [PubMed: 23248172]

Tartaglia LA, Dembski M, Weng X, Deng N, Culpepper J, Devos R, et al. Identification and expression cloning of a leptin receptor, OB-R. Cell. 1995; 83:1263-1271. [PubMed: 8548812]

Taylor JR, Dietrich E, Powell J. Lorcaserin for weight management. Diabetes Metab Syndr Obes. 2013; 6:209-216. [PubMed: 23788837]

Tedesco L, Valerio A, Cervino C, Cardile A, Pagano C, Vettor R, et al. Cannabinoid type 1 receptor blockade promotes mitochondrial biogenesis through endothelial nitric oxide synthase expression in white adipocytes. Diabetes. 2008; 57:2028-2036. [PubMed: 18477809]

Ten Kulve JS, Veltman DJ, van Bloemendaal L, Barkhof F, Drent ML, Diamant M, et al. Liraglutide Reduces CNS Activation in Response to Visual Food Cues Only After Short-term Treatment in Patients With Type 2 Diabetes. Diabetes Care. 2015 [Epub ahead of print].

Theander-Carrillo C, Wiedmer P, Cettour-Rose P, Nogueiras R, Perez-Tilve D, Pfluger P, et al. Ghrelin action in the brain controls adipocyte metabolism. J Clin Invest. 2006; 116:1983-1993. [PubMed: 16767221]

Thomas C, Gioiello A, Noriega L, Strehle A, Oury J, Rizzo G, et al. TGR5-mediated bile acid sensing controls glucose homeostasis. Cell Metab. 2009; 10:167-177. [PubMed: 19723493]

Thomsen C, Rasmussen O, Lousen T, Holst JJ, Fenselau S, Schrezenmeir J, et al. Differential effects of saturated and monounsaturated fatty acids on postprandial lipemia and incretin responses in healthy subjects. Am J Clin Nutr. 1999; 69:1135-1143. [PubMed: 10357731]

Torgerson JS, Hauptman J, Boldrin MN, Sjostrom L. XENical in the prevention of diabetes in obese subjects (XENDOS) study: a randomized study of orlistat as an adjunct to lifestyle changes for the prevention of type 2 diabetes in obese patients. Diabetes Care. 2004; 27:155-161. [PubMed: 14693982]

Torres SJ, Nowson CA. Relationship between stress, eating behavior, and obesity. Nutrition. 2007; 23:887-894. [PubMed: 17869482]

Trivedi P, Yu H, MacNeil DJ, Van der Ploeg LH, Guan XM. Distribution of orexin receptor mRNA in the rat brain. FEBS Lett. 1998; 438:71-75. [PubMed: 9821961]

Tsuchida A, Yamauchi T, Ito Y, Hada Y, Maki T, Takekawa S, et al. Insulin/Foxo1 pathway regulates expression levels of adiponectin receptors and adiponectin sensitivity. J Biol Chem. 2004; 279:30817-30822. [PubMed: 15123605]

Veldhuis JD, Iranmanesh A, Ho KK, Waters MJ, Johnson ML, Lizarralde G. Dual defects in pulsatile growth hormone secretion and clearance subserve the hyposomatotropism of obesity in man. $\mathrm{J}$ Clin Endocrinol Metab. 1991; 72:51-59. [PubMed: 1986027] 
Vergoni AV, Bertolini A, Wikberg JE, Schioth HB. Corticotropin-releasing factor (CRF) induced anorexia is not influenced by a melanocortin 4 receptor blockage. Peptides. 1999; 20:509-513. [PubMed: 10458522]

Verty AN, Lockie SH, Stefanidis A, Oldfield BJ. Anti-obesity effects of the combined administration of CB1 receptor antagonist rimonabant and melanin-concentrating hormone antagonist SNAP-94847 in diet-induced obese mice. Int J Obes (Lond). 2013; 37:279-287. [PubMed: 22473329]

Volkow ND, O'Brien CP. Issues for DSM-V: should obesity be included as a brain disorder? Am J Psychiatry. 2007; 164:708-710. [PubMed: 17475727]

Volkow ND, Wang GJ, Fowler JS, Logan J, Jayne M, Franceschi D, et al. "Nonhedonic" food motivation in humans involves dopamine in the dorsal striatum and methylphenidate amplifies this effect. Synapse. 2002; 44:175-180. [PubMed: 11954049]

Volkow ND, Wang GJ, Fowler JS, Telang F. Overlapping neuronal circuits in addiction and obesity: evidence of systems pathology. Philos Trans R Soc Lond B Biol Sci. 2008; 363:3191-3200. [PubMed: 18640912]

Volkow ND, Wang GJ, Fowler JS, Tomasi D, Baler R. Food and drug reward: overlapping circuits in human obesity and addiction. Curr Top Behav Neurosci. 2012; 11:1-24. [PubMed: 22016109]

Volkow ND, Wang GJ, Telang F, Fowler JS, Goldstein RZ, Alia-Klein N, et al. Inverse association between BMI and prefrontal metabolic activity in healthy adults. Obesity (Silver Spring). 2009; 17:60-65. [PubMed: 18948965]

Volkow ND, Wise RA. How can drug addiction help us understand obesity? Nat Neurosci. 2005; 8:555-560. [PubMed: 15856062]

von der Goltz C, Koopmann A, Dinter C, Richter A, Rockenbach C, Grosshans M, et al. Orexin and leptin are associated with nicotine craving: a link between smoking, appetite and reward. Psychoneuroendocrinology. 2010; 35:570-577. [PubMed: 19828259]

Vrieze A, Van Nood E, Holleman F, Salojarvi J, Kootte RS, Bartelsman JF, et al. Transfer of intestinal microbiota from lean donors increases insulin sensitivity in individuals with metabolic syndrome. Gastroenterology. 2012; 143:913-916. e917. [PubMed: 22728514]

Wang C, Bomberg E, Billington C, Levine A, Kotz CM. Brain-derived neurotrophic factor in the hypothalamic paraventricular nucleus reduces energy intake. Am J Physiol Regul Integr Comp Physiol. 2007; 293:R1003-1012. [PubMed: 17581841]

Wang GJ, Tomasi D, Volkow ND, Wang R, Telang F, Caparelli EC, et al. Effect of combined naltrexone and bupropion therapy on the brain's reactivity to food cues. Int J Obes (Lond). 2014; 38:682-688. [PubMed: 23924756]

Wang GJ, Volkow ND, Fowler JS. The role of dopamine in motivation for food in humans: implications for obesity. Expert Opin Ther Targets. 2002; 6:601-609. [PubMed: 12387683]

Wang GJ, Volkow ND, Logan J, Pappas NR, Wong CT, Zhu W, et al. Brain dopamine and obesity. Lancet. 2001; 357:354-357. [PubMed: 11210998]

Ward HG, Nicklous DM, Aloyo VJ, Simansky KJ. Mu-opioid receptor cellular function in the nucleus accumbens is essential for hedonically driven eating. Eur J Neurosci. 2006; 23:1605-1613. [PubMed: 16553624]

Warwick ZS, Weingarten HP. Determinants of high-fat diet hyperphagia: experimental dissection of orosensory and postingestive effects. Am J Physiol. 1995; 269:R30-37. [PubMed: 7631900]

Weissman NJ, Tighe JF Jr. Gottdiener JS, Gwynne JT. An assessment of heart-valve abnormalities in obese patients taking dexfenfluramine, sustained-release dexfenfluramine, or placebo. SustainedRelease Dexfenfluramine Study Group. N Engl J Med. 1998; 339:725-732. [PubMed: 9731088]

Wellman PJ. Norepinephrine and the control of food intake. Nutrition. 2000; 16:837-842. [PubMed: 11054588]

Whitehouse F, Kruger DF, Fineman M, Shen L, Ruggles JA, Maggs DG, et al. A randomized study and open-label extension evaluating the long-term efficacy of pramlintide as an adjunct to insulin therapy in type 1 diabetes. Diabetes Care. 2002; 25:724-730. [PubMed: 11919132]

WHO Fact sheet N³11. 2015. http://www.who.int/mediacentre/factsheets/fs311/en/ 
Willesen MG, Kristensen P, Romer J. Co-localization of growth hormone secretagogue receptor and NPY mRNA in the arcuate nucleus of the rat. Neuroendocrinology. 1999; 70:306-316. [PubMed: 10567856]

Williams DL. Neural integration of satiation and food reward: role of GLP-1 and orexin pathways. Physiol Behav. 2014; 136:194-199. [PubMed: 24650552]

Williams T, Berelowitz M, Joffe SN, Thorner MO, Rivier J, Vale W, et al. Impaired growth hormone responses to growth hormone-releasing factor in obesity. A pituitary defect reversed with weight reduction. N Engl J Med. 1984; 311:1403-1407. [PubMed: 6436706]

Williams KW, Elmquist JK. From neuroanatomy to behavior: central integration of peripheral signals regulating feeding behavior. Nat Neurosci. 2012; 15(10):1350-1355. [PubMed: 23007190]

Williams G, Gill JS, Lee YC, Cardoso HM, Okpere BE, Bloom SR. Increased neuropeptide Y concentrations in specific hypothalamic regions of streptozocin-induced diabetic rats. Diabetes. 1989; 38:321-327. [PubMed: 2563712]

Willie JT, Chemelli RM, Sinton CM, Yanagisawa M. To eat or to sleep?. Orexin in the regulation of feeding and wakefulness. Annu Rev Neurosci. 2001; 24:429-458. [PubMed: 11283317]

Wittmann G, Deli L, Kallo I, Hrabovszky E, Watanabe M, Liposits Z, et al. Distribution of type 1 cannabinoid receptor (CB1)-immunoreactive axons in the mouse hypothalamus. J Comp Neurol. 2007; 503:270-279. [PubMed: 17492633]

Woods SC, Decke E, Vasselli JR. Metabolic hormones and regulation of body weight. Psychol Rev. 1974; 81:26-43. [PubMed: 4812879]

Woods SC, Seeley RJ, Baskin DG, Schwartz MW. Insulin and the blood-brain barrier. Curr Pharm Des. 2003; 9:795-800. [PubMed: 12678878]

Wotherspoon G, Fox A, McIntyre P, Colley S, Bevan S, Winter J. Peripheral nerve injury induces cannabinoid receptor 2 protein expression in rat sensory neurons. Neuroscience. 2005; 135:235245. [PubMed: 16084654]

Wren AM, Small CJ, Ward HL, Murphy KG, Dakin CL, Taheri S, et al. The novel hypothalamic peptide ghrelin stimulates food intake and growth hormone secretion. Endocrinology. 2000; 141:4325-4328. [PubMed: 11089570]

Wynne K, Park AJ, Small CJ, Patterson M, Ellis SM, Murphy KG, et al. Subcutaneous oxyntomodulin reduces body weight in overweight and obese subjects: a double-blind, randomized, controlled trial. Diabetes. 2005; 54:2390-2395. [PubMed: 16046306]

Wynne K, Stanley S, McGowan B, Bloom S. Appetite control. J Endocrinol. 2005; 184:291-318. [PubMed: 15684339]

Xu B, Goulding EH, Zang K, Cepoi D, Cone RD, Jones KR, et al. Brain-derived neurotrophic factor regulates energy balance downstream of melanocortin-4 receptor. Nat Neurosci. 2003; 6:736742. [PubMed: 12796784]

Yamauchi T, Kamon J, Ito Y, Tsuchida A, Yokomizo T, Kita S, et al. Cloning of adiponectin receptors that mediate antidiabetic metabolic effects. Nature. 2003; 423:762-769. [PubMed: 12802337]

Yang WS, Lee WJ, Funahashi T, Tanaka S, Matsuzawa Y, Chao CL, et al. Weight reduction increases plasma levels of an adipose-derived anti-inflammatory protein, adiponectin. J Clin Endocrinol Metab. 2001; 86:3815-3819. [PubMed: 11502817]

Yanovski SZ, Yanovski JA. Long-term drug treatment for obesity: a systematic and clinical review. JAMA. 2014; 311:74-86. [PubMed: 24231879]

Yeh JR, Ju R, Brdlik CM, Zhang W, Zhang Y, Matyskiela ME, et al. Targeted gene disruption of methionine aminopeptidase 2 results in an embryonic gastrulation defect and endothelial cell growth arrest. Proc Natl Acad Sci U S A. 2006; 103:10379-10384. [PubMed: 16790550]

Yeo GS, Farooqi IS, Aminian S, Halsall DJ, Stanhope RG, O'Rahilly S. A frameshift mutation in MC4R associated with dominantly inherited human obesity. Nat Genet. 1998; 20:111-112. [PubMed: 9771698]

Yin HH, Knowlton BJ, Balleine BW. Lesions of dorsolateral striatum preserve outcome expectancy but disrupt habit formation in instrumental learning. Eur J Neurosci. 2004; 19:181-189. [PubMed: 14750976] 
Zhang J, Frassetto A, Huang RR, Lao JZ, Pasternak A, Wang SP, et al. The mu-opioid receptor subtype is required for the anorectic effect of an opioid receptor antagonist. Eur J Pharmacol. 2006; 545:147-152. [PubMed: 16876155]

Zhang M, Balmadrid C, Kelley AE. Nucleus accumbens opioid, GABAergic, and dopaminergic modulation of palatable food motivation: contrasting effects revealed by a progressive ratio study in the rat. Behav Neurosci. 2003; 117:202-211. [PubMed: 12708516]

Zhang M, Gosnell BA, Kelley AE. Intake of high-fat food is selectively enhanced by mu opioid receptor stimulation within the nucleus accumbens. J Pharmacol Exp Ther. 1998; 285:908-914. [PubMed: 9580643]

Zhang Y, Proenca R, Maffei M, Barone M, Leopold L, Friedman JM. Positional cloning of the mouse obese gene and its human homologue. Nature. 1994; 372:425-432. [PubMed: 7984236]

Zheng H, Patterson LM, Berthoud HR. Orexin-A projections to the caudal medulla and orexin-induced c-Fos expression, food intake, and autonomic function. J Comp Neurol. 2005; 485:127-142. [PubMed: 15776447]

Zhi J, Melia AT, Funk C, Viger-Chougnet A, Hopfgartner G, Lausecker B, et al. Metabolic profiles of minimally absorbed orlistat in obese/overweight volunteers. J Clin Pharmacol. 1996; 36:10061011. [PubMed: 8973989]

Zorrilla EP, Iwasaki S, Moss JA, Chang J, Otsuji J, Inoue K, et al. Vaccination against weight gain. Proc Natl Acad Sci U S A. 2006; 103:13226-13231. [PubMed: 16891413] 


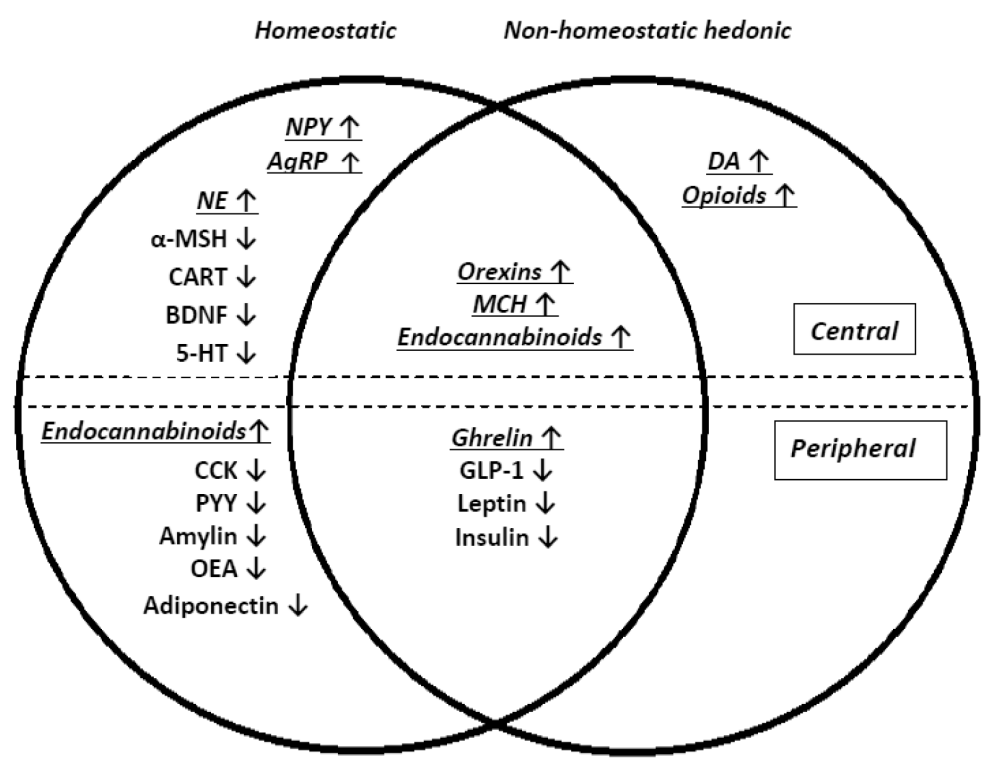

Figure 1.

Homeostatic and non-homeostatic hedonic factors that regulate food intake. Central and peripheral signals are provided above and below, respectively, the double line. " $\uparrow$ ” indicates increases in food intake induced by orexigenic factors (also represented by the underline). “ $\downarrow$ ” indicates decreases in food intake induced by anorexigenic factors. Increased orexigenic signaling facilitates the development of obesity and increased anorexigenic signaling inhibits the development of obesity. 


\section{Table 1}

\section{Approved Anti-obesity Drugs}

\begin{tabular}{|c|c|}
\hline Drug & Mechanism of Action \\
\hline $\begin{array}{l}\text { Phentermine } \\
\text { (Adipex-Pß) }\end{array}$ & $\begin{array}{l}\text { NE transporter inhibitor; Appetite suppression mediated by } \\
\text { activation of POMC neurons in the arcuate nucleus }\end{array}$ \\
\hline $\begin{array}{l}\text { Orlistat } \\
\text { (Xenicalß) }\end{array}$ & $\begin{array}{l}\text { Gastric- and pancreatic-lipase inhibitor; Reduces absorption of } \\
\text { dietary fat }\end{array}$ \\
\hline $\begin{array}{l}\text { Lorcaserin } \\
\left.\text { (Belviq }{ }^{\circledR}\right)\end{array}$ & Selective $5-\mathrm{HT}_{2 \mathrm{C}}$ agonist; Promotes satiety \\
\hline $\begin{array}{l}\text { Phentermine } \\
\text { and Topiramate } \\
\text { (Qsymia } ®)\end{array}$ & $\begin{array}{l}\text { Phentermine: NE transporter inhibitor; Appetite suppression } \\
\text { mediated by activation of POMC neurons in the arcuate nucleus } \\
\text { Topiramate: GABA agonist; Appetite suppression may be due to } \\
\text { modulation of voltage-gated ion channels, increased activity at } \\
\text { GABA-A receptors and/or inhibition of AMPA/kainite glutamate } \\
\text { receptors }\end{array}$ \\
\hline $\begin{array}{l}\text { Naltrexone and } \\
\text { Bupropion } \\
\text { (Contrave }{ }^{\circledR} \text { ) }\end{array}$ & $\begin{array}{l}\text { Naltrexone: Opioid receptor antagonist; Prevents } \beta \text {-endorphin-mediated } \\
\text { negative feedback on a-MSH release } \\
\text { Bupropion: DA and NE transporter inhibitor; Stimulates } \\
\text { hypothalamic POMC neurons that release a-MSH resulting in } \\
\text { decreased food intake and increased energy expenditure }\end{array}$ \\
\hline $\begin{array}{l}\text { Liraglutide } \\
\text { (Saxenda } ®)\end{array}$ & GLP-1 agonist; Decreases appetite \\
\hline
\end{tabular}




\section{Table 2}

\section{Anti-obesity Therapeutics in Clinical Development}

\begin{tabular}{|c|c|c|}
\hline Drug & Mechanism of Action & Clinical Phase \\
\hline Tesofensine & 5-HT, DA and NE transporter inhibitor & Phase III \\
\hline $\begin{array}{l}\text { Bupropion } \\
\text { and } \\
\text { Zonisamide } \\
\left.\text { (Empatic }{ }^{\circledR}\right)\end{array}$ & $\begin{array}{l}\text { Bupropion: DA and NE transporter inhibitor; } \\
\text { Stimulates hypothalamic POMC neurons releasing } \\
\text { a-MSH that leads to decreased food intake and } \\
\text { increased energy expenditure } \\
\text { Zonisamide: Carbonic anhydrase inhibitor; } \\
\text { Reduces lipogenesis }\end{array}$ & Phase III \\
\hline Exenatide & GLP-1 agonist; Decreases appetite & Phase III \\
\hline Cetilistat & $\begin{array}{l}\text { Gastric- and pancreatic-lipase inhibitor; } \\
\text { Reduces absorption of dietary fat }\end{array}$ & Phase III \\
\hline Beloranib & $\begin{array}{l}\text { MetAP2 inhibitor; Reduces fat biosynthesis, } \\
\text { stimulates fat oxidation and lipolysis }\end{array}$ & Phase II \\
\hline$R M-493$ & $\begin{array}{l}\text { Selective MC4R agonist; Decreases appetite and } \\
\text { increases metabolism }\end{array}$ & Phase II \\
\hline KD026 & $\begin{array}{l}\text { Selective intestinal microsomal transfer protein } \\
\text { inhibitor; Reduces fat absorption }\end{array}$ & Phase II \\
\hline $\begin{array}{l}\text { Remogliflozin } \\
\text { etabonate }\end{array}$ & $\begin{array}{l}\text { Selective inhibitor of sodium-glucose } \\
\text { cotransporter } 2\end{array}$ & Phase II \\
\hline
\end{tabular}




\section{Table 3}

Anti-obesity Therapeutic Candidates and Targets

\begin{tabular}{|c|c|}
\hline Drug/Target & Mechanism of Action \\
\hline$P R X-07034$ & $5-\mathrm{HT}_{6}$ antagonist \\
\hline $\begin{array}{l}\text { Rimonabant and } \\
S N A P-94847\end{array}$ & $\begin{array}{l}\text { Rimonabant: CB1 antagonist } \\
\text { SNAP-94847: MCH1 antagonist }\end{array}$ \\
\hline GSK1521498 & $\begin{array}{l}\text { Selective } \mu \text {-opioid receptor antagonist; } \\
\text { Decreases binge eating of palatable foods }\end{array}$ \\
\hline RM-493 and Liraglutide & $\begin{array}{l}\text { RM-493: MC4R agonist } \\
\text { Liraglutide: GLP-1 agonist }\end{array}$ \\
\hline $\begin{array}{l}\text { Pramlintide and } \\
\text { Metreleptin }\end{array}$ & $\begin{array}{l}\text { Pramlintide: Amylin analog; Restores leptin sensitivity } \\
\text { Metreleptin: Recombinant human leptin; Decreases } \\
\text { food intake and increases energy expenditure }\end{array}$ \\
\hline $\begin{array}{l}\text { Glucagon and } G L P-1 \\
\text { receptor co-agonism }\end{array}$ & Decreases appetite and increases energy expenditure \\
\hline Growth hormone & Increases energy expenditure \\
\hline $\begin{array}{l}\text { Bile acid receptors: } F X R \\
\text { and } T G R 5\end{array}$ & $\begin{array}{l}\text { Intestinal FXR: Regulates energy expenditure } \\
\text { TGR5: Decreases appetite and increases energy } \\
\text { expenditure }\end{array}$ \\
\hline
\end{tabular}

